\title{
3D Medical Image Reconstruction On Digital Breast Tomosynthesis
}

Siemens S.A. - Healthcare Sector

$2008 / 2009$

\author{
Isabel Catarina Castro Duarte \\ Integrated Master in Biomedical Engineering
}

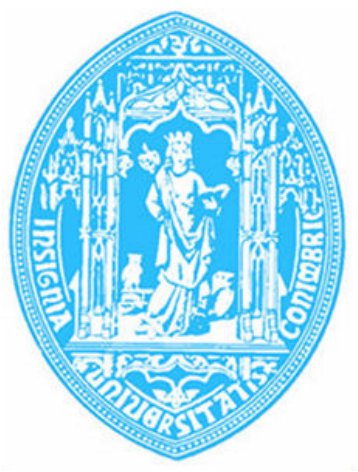

Physics Department,

Faculty of Sciences and Technology of University of Coimbra 



\title{
3D Medical Image Reconstruction On Digital Breast Tomosynthesis
}

\author{
Siemens S.A. - Healthcare Sector
}

$2008 / 2009$

2004107215 Isabel Catarina Castro Duarte Integrated Master in Biomedical Engineering

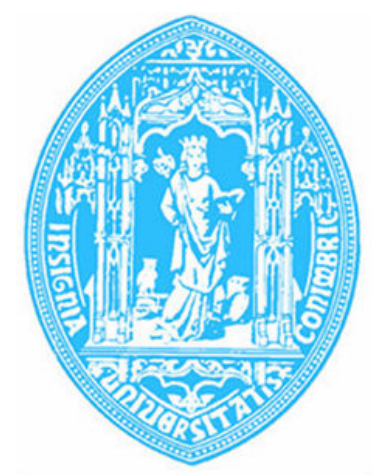

Physics Department

September 2009

Adviser from FCTUC: Professor Doctor José Silvestre Silva

Supervisor from Siemens, S.A.: Engineer Filipe Janela 
The present dissertation contains strictly confidential information, so it can not be copied, transmitted or disseminated, in part or in whole without the express written consent of the author and the Siemens Healthcare Sector. 
Aos meus pais 


\section{Acknowledgments}

I would like to express my gratitude for those that made the present work feasible and collaborated in the conclusion of this work.

Firstly, I wish to thank to Engineer Filipe Janela and to the Siemens S.A. for the opportunity given to carry out this work under all the conditions that, otherwise, I would not have. I had several opportunities to learn and to improve my knowledge, so I cannot be more grateful.

I also wish to thank to my adviser from the University of Coimbra, Professor Doctor José Silvestre Silva, for the help and all the interest on this project. Professor was the one who always remembered that I was far from Coimbra and provided me information that otherwise would not reach me. Professor José Silva has ensured various times my interests in the University. I am very thankful for all.

I am extremely grateful to the Engineers Liliana Caldeira and Filipe Soares. They were the mentors of this project and they were the supervisors that always accompanied and helped in everything. Without them this work would not be the same.

I want to express thanks to the Milipeia administrators, that allowed the utilization of the computer cluster to perform the Monte Carlo simulations, an extreme important step in this work.

I could not forget my colleagues of the Costumer Service and Software and Engineering Group, in particular the Engineer António Martins. Thanks for all help, tips and patience...

A special thanks to Dr. Celina Lourenço, for the evenings reading this thesis, for the help on the integration on the company, for the constant support and interest in this work and for all the support.

I also want to thank to Doctor Nuno Matela for the help and clarifications about the image reconstruction. There were tips that make the difference.

I am grateful to Engineer Inês Sousa, for the advices and for lending me her computer, several times.

I also have to refer the Professor Doctor Isabel Lopes and Professor Doctor Miguel Morgado that for theirs interest and dynamism make the course of Biomedical Engineering in Coimbra a success. 
A special thanks to my colleagues and friends: Catarina, Dora and Bianca, and the Masters João and Leite. They made it all bearable.

To my parents, my mother Conceição and my father António, who I dedicate this thesis. I wish to thank for all the support in the last five years, they were, in fact, who made all this possible. I also thank to my brothers Nuno and Ricardo by the support. I could not forget Andreia and Filipa: thanks for the friendship.

Finally, I thank to my unconditional supporter: Tiago. 


\section{Abstract}

The breast cancer is a deadly disease and one of the types with higher incidence. It is asymptomatic at earlier stages, but it has a high survival rate at the earliest stages. These characteristics make the screening and the accurate diagnostic important issues. With the aim of reduce the damage caused by breast diseases, new image techniques have arisen and the current techniques are in constantly improving. Digital Breast Tomosynthesis appears as an improvement to the mammography, which is considered the gold standard. This technique will improve the sensibility and specificity of mammography and will allow the 3D visualization without overlapping tissues.

Different types of tomosynthesis reconstruction methods have been explored. However, these algorithms are not totally developed neither optimized. The aim of the present project is the implementation and the assessment of image reconstruction algorithms. With this aim, simulated data will be used to perform and assess the tridimensional reconstruction. The simulations will be performed on a platform of simulation based on Monte Carlo methods, GATE, using an analytical phantom and a realistic breast phantom, the NCAT phantom.

The reconstruction step was performed, implementing the filtered back projection, the maximum-likelihood expectation-maximization and the algebraic reconstruction technique on the MATLAB software.

The three algorithms presented comparable results. The results of the 3D image reconstruction allow the discrimination of smaller structures, the spheres, than could not be differentiated on the simple projections that result from the simulations. This proves that the accuracy of Digital Breast Tomosynthesis can be better than the mammography. The FBP algorithm presented more blurring images than the ML-EM and ART algorithms. However, it was the more capable to localize the structures on the 3D space, including the smaller details.

Key words:

Digital Breast Tomosynthesis, Monte Carlo Simulations, Filtered Back Projection, Algebraic Reconstruction Technique, Maximum-Likelihood Expectation-Maximization. 


\section{Resumo}

O cancro da mama é uma patologia mortal e é um dos tipos de cancro com maior incidência. O cancro da mama é assintomático nos estádios iniciais, mas tem elevadas taxa de sobrevivência quando detectado precocemente. Isto torna o rastreio e o diagnóstico preciso questões de grande importância. Com o objectivo de reduzir as consequências das doenças da mama, têm surgido novas técnicas de imagem e as existentes estão em constante melhoramento. A Tomossíntese Digital da Mama (TDM) surge como um melhoramento da mamografia, que é considerada como prática de eleição. A TDM irá aumentar a sensibilidade e especificidade da mamografia e permitirá a visualização tridimensional (3D) sem os problemas da sobreposição dos tecidos.

Tipos diferentes de métodos de reconstrução têm sido explorados. Contudo, esses algoritmos não estão totalmente desenvolvidos nem optimizados para este caso. 0 objectivo do presente projecto é a implementação e a avaliação de algoritmos de reconstrução. Serão utilizadas imagens simuladas para implementar e avaliar os algoritmos. Para tal, será utilizado um programa baseado em métodos de Monte Carlo, o GATE, usando fantomas analíticos e um mais realista, o fantoma NCAT.

O passo da reconstrução será realizado com a implementação do FBP (do inglês filtered back projection), o ML-EM (do inglês maximum-likelihood expectation-maximization) e o ART (do inglês algebraic reconstruction technique) no programa MATLAB.

Os três algoritmos apresentaram resultados comparáveis. Os resultados da reconstrução 3D permitem a discriminação das estruturas mais pequenas que não são diferenciadas nas projecções que resultam das simulações. Isto prova que a precisão da TDM pode ser melhor que a da mamografia. O algoritmo FBP apresentou imagens com menos nítidas que o ML-EM e o ART. Contudo, o FBP foi o mais eficaz a localizar as estruturas tridimensionalmente, incluindo os pequenos detalhes.

\section{Palavras Chave: $\quad$ Tomossíntese Digital da Mama,}

Simulações de Monte Carlo,

Filtered Back Projection,

Algebraic Reconstruction Technique,

Maximum-Likelihood Expectation-Maximization. 


\section{Index}

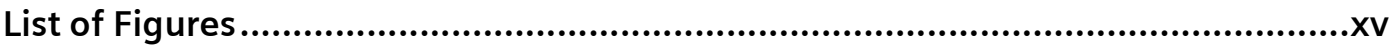

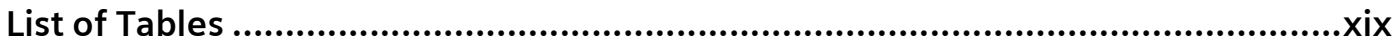

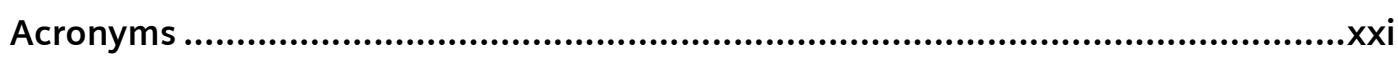

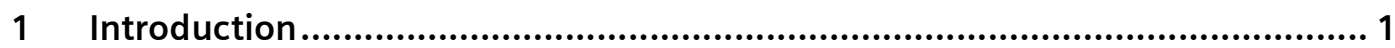

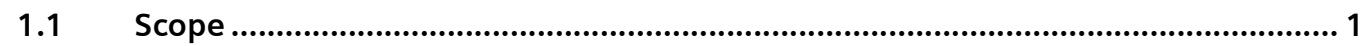

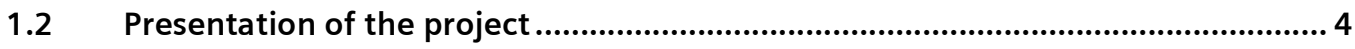

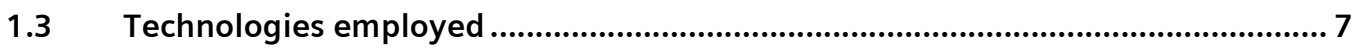

1.3.1 Geant4 Application for Tomographic Emission .............................................. 7

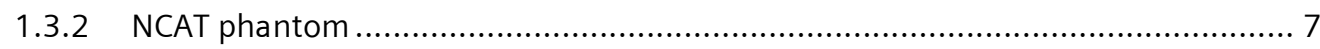

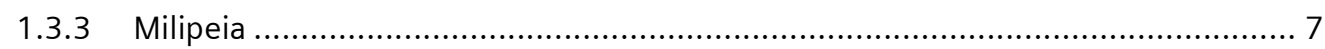

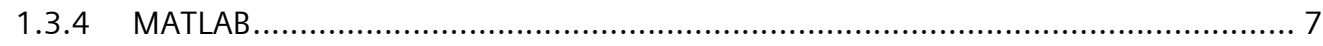

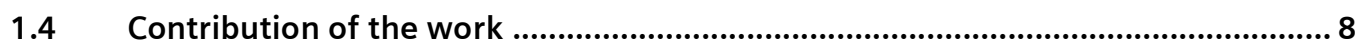

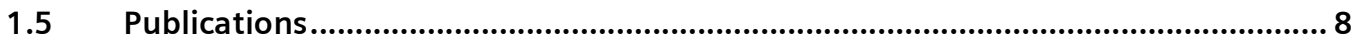

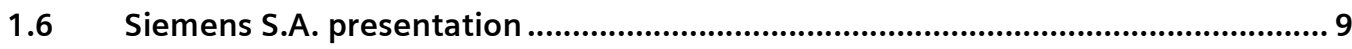

$1.7 \quad$ Organization of the chapters .................................................................................... 10

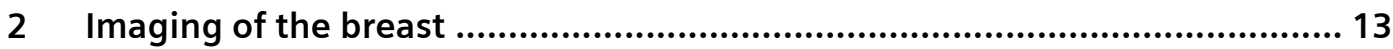

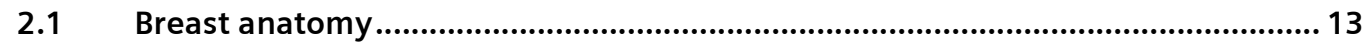

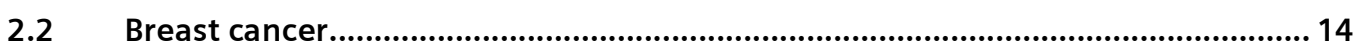

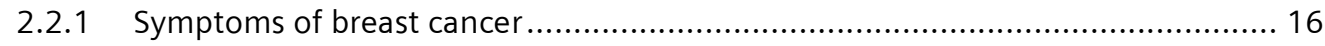

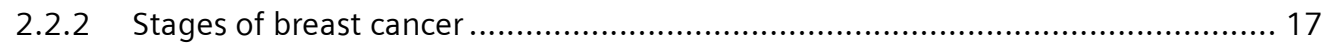

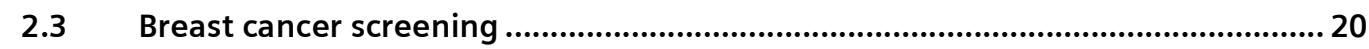

2.4 Current techniques for breast imaging ................................................................... 20

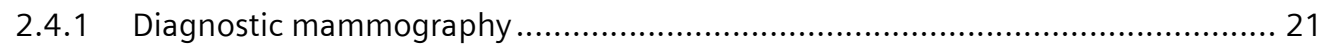

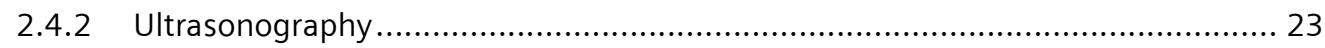

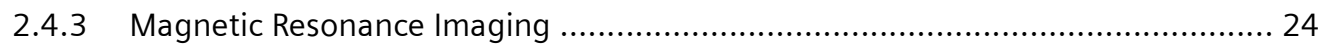

$2.5 \quad$ Others techniques for imaging of the breast .......................................................... 25

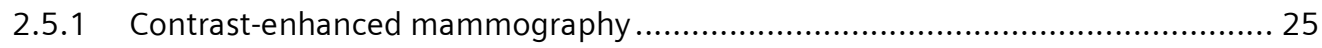




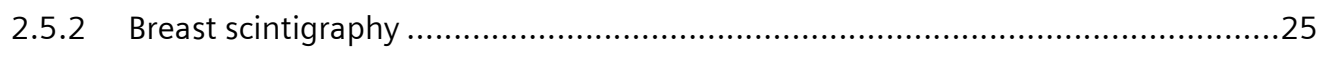

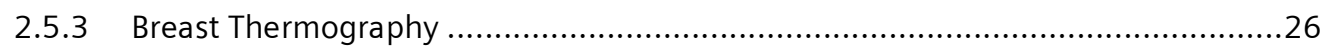

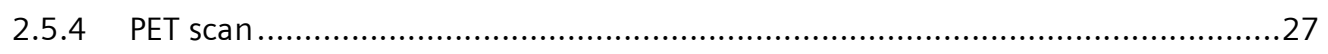

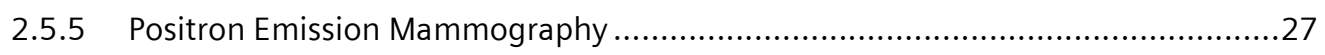

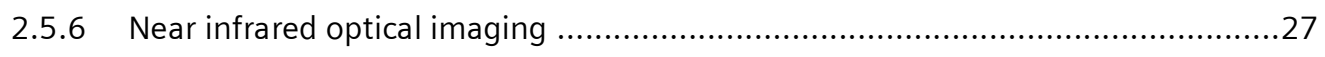

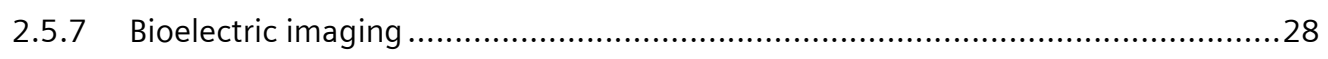

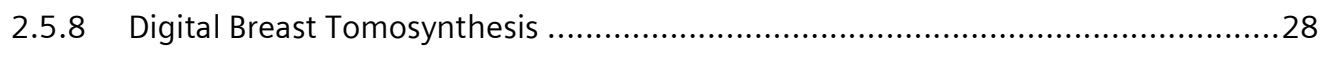

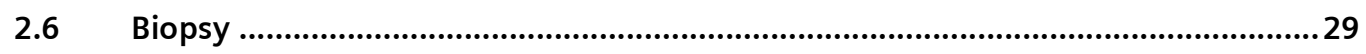

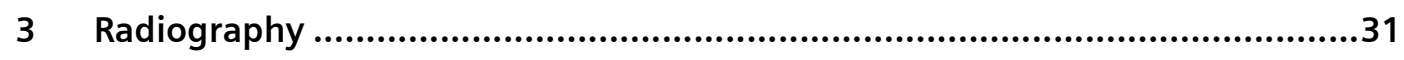

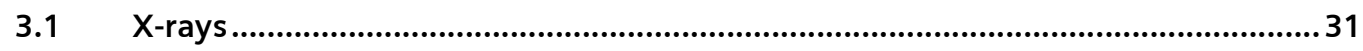

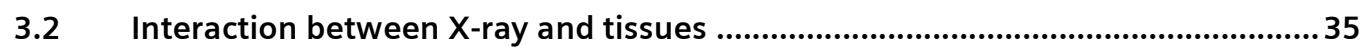

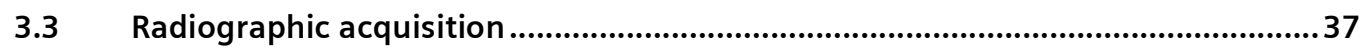

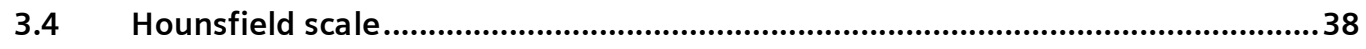

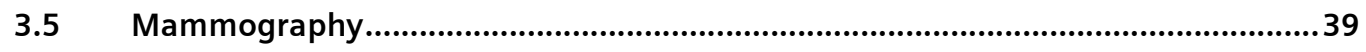

B.6 Digital Breast Tomosynthesis.........................................................................42

4 Monte Carlo Simulations .................................................................47

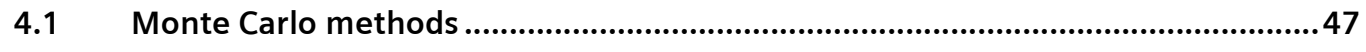

4.1.1 General-purpose source codes based on Monte Carlo methods.........................49

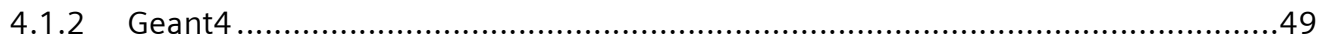

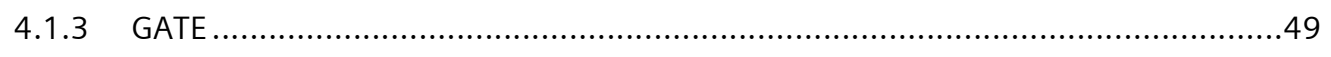

4.2 State-of-art of MC simulation in mammography ..........................................50

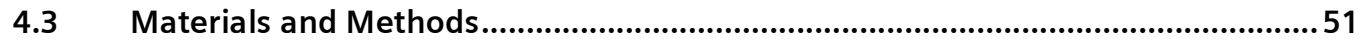

4.3.1 Mammographic unit ............................................................................ 51

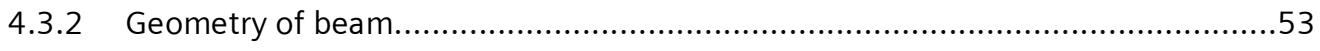

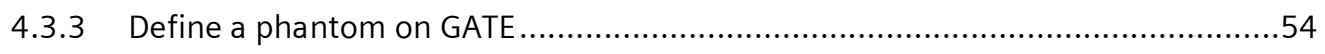

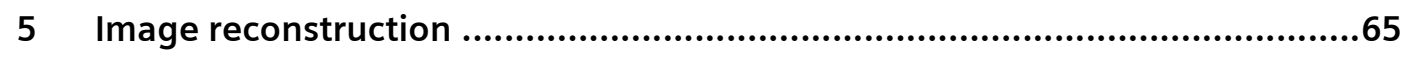

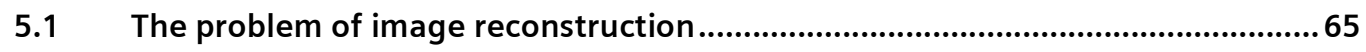

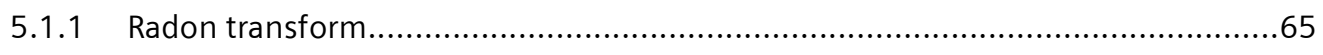

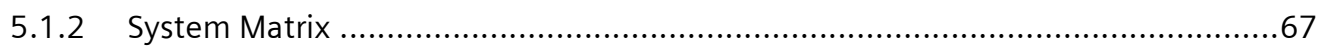


5.2 State-of-art of 3D image reconstruction in tomosynthesis .......................................68

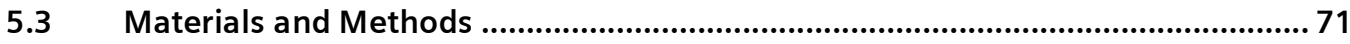

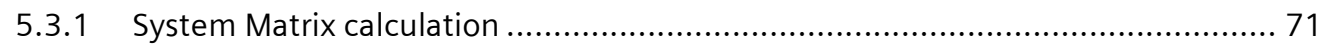

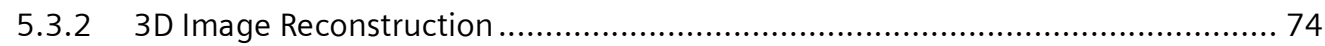

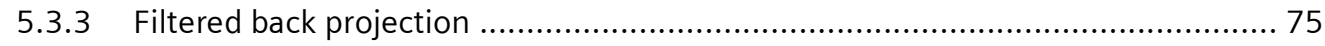

5.3.4 Algebraic Reconstruction Technique ................................................................. 76

5.3.5 Maximum-Likelihood Expectation-Maximization.................................................... 78

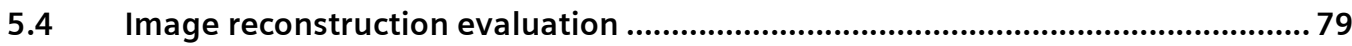

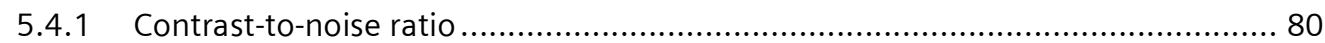

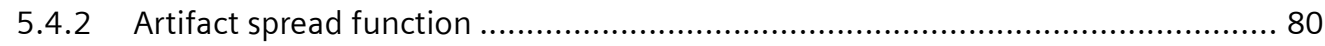

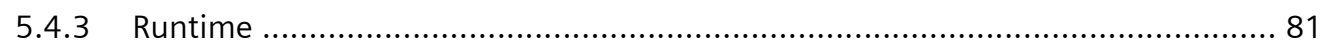

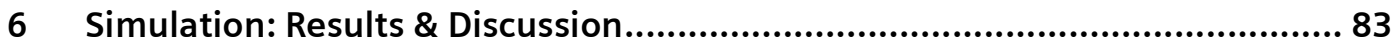

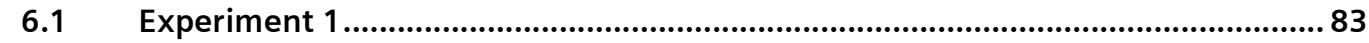

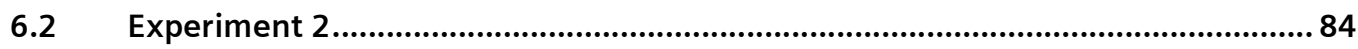

$6.3 \quad$ Experiment 3

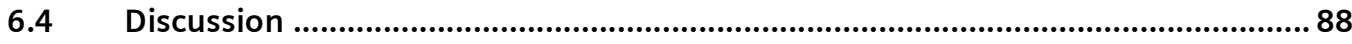

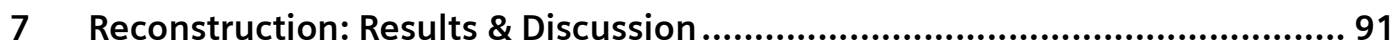

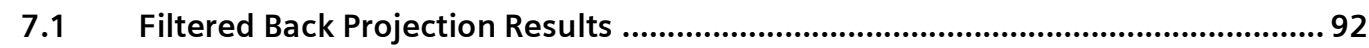

7.2 Algebraic Reconstruction Technique Results ...........................................................96

7.3 Maximum-Likelihood Expectation-Maximization Results ......................................100

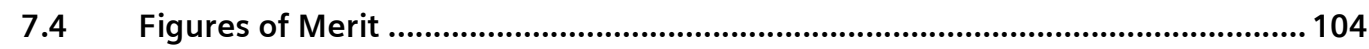

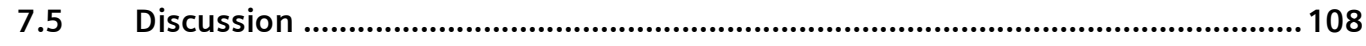

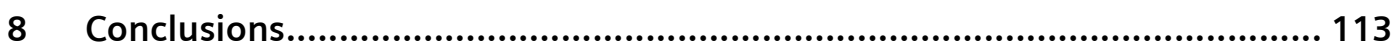

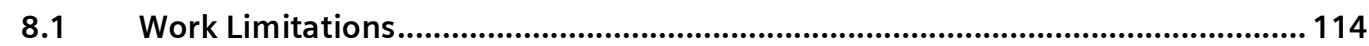

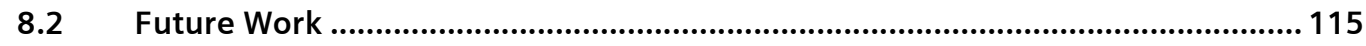

8.3 Final Work Assessment .................................................................................... 117

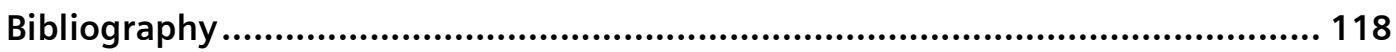

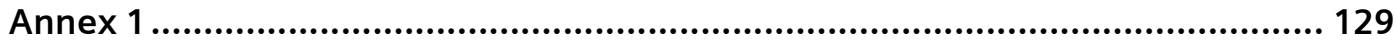

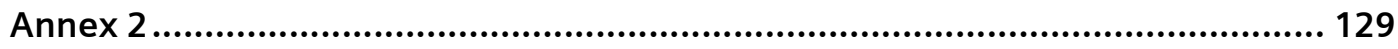




\section{List of Figures}

Figure 1.1 International variation in breast cancer incidence rates. [7] .................... 2

Figure 2.1 Structure of a normal breast, adapted from. [22] ............................... 14

Figure 2.2 Picture of a breast, explaining the difference between in situ and invasive ductal carcinoma. In the last one, it is visible the invasion of the surrounding tissue by tumour ductal cells. Adapted from [25]....................................................... 16

Figure 2.3 Appearance of some symptoms of breast cancer. Adapted from [27] ...... 17

Figure 2.4 The most common mammographic views: cranio-caudal (CC) mammographic view, medio-lateral oblique (MLO) mammographic view, latero medial (LM) mammographic view, medio-lateral (ML) mammographic view..................... 21

Figure 2.5 Representation of a mammographic acquisition. Adapted from [27] ....... 22

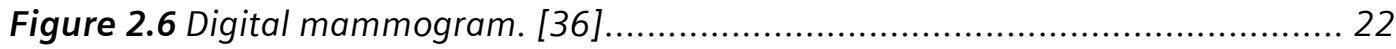

Figure 2.7 Ultrasound image of breast evidencing a fibroadenoma. [36] ................. 23

Figure 2.8 Resonance magnetic image of the breasts. [36] .............................. 24

Figure 2.9 Scintigram of breast showing an intense soft tissue uptake in a carcinoma

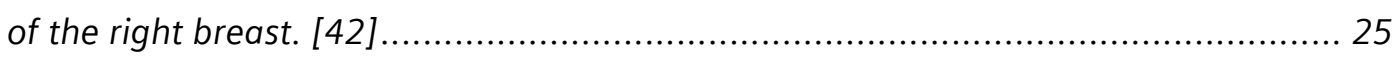

Figure 2.10 Image of corporal temperature acquired by thermography. [43] ........... 26

Figure 2.11 PET image evidencing a region of high uptake. [44] ......................... 27

Figure 2.12 Schema of the acquisition of images in DBT, illustrating the movements of the $X$-ray tube around the breast. .................................................................... 28

Figure 2.13 Picture showing a biopsy for breast. [36]...................................... 29

Figure 3.1 Electromagnetic spectrum, illustrating the wavelength and the wave

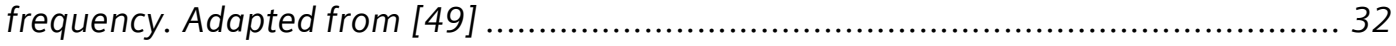

Figure 3.2 X-ray tube and its compounds. Adapted from [51] ........................... 32

Figure 3.3 Generation of Bremsstrahlung radiation a) Generation of characteristic

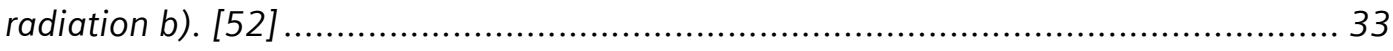

Figure $3.4 X$-ray spectrum produced by a Tungsten target. Adapted from [53] ......... 34 
Figure 3.5 Illustration showing that the penumbra on the image has its origin on the increase of focal area. Adapted from [56]

Figure 3.6 Graphic of total mass attenuation coefficients for soft tissues, resulting from the sum of the diverse forms of interaction between radiation and soft tissues. Adapted from [58] 36

Figure 3.7 Hounsfield units range for different tissues. [62] .... 38

Figure 3.8 Mean values of the linear attenuation coefficient of invasive ductal carcinoma, fibrous and fat tissue. [63]

Figure 3.9 Diagram illustrating the tomosynthesis system, where the $x$-ray tube moves around an arc to take the projections. 42

Figure 3.10 Schema of the image acquisition on different angles of the same object. The reconstruction of the various projections will allow the distinction between different structures.

Figure 3.11 Image processing.

Figure 4.1 MAMMOMAT Inspiration design. [36] ....

Figure 4.2 Diagram representing the geometry of the system, explaining the polar and the azimuthal angle.

Figure 4.3 System completed in CTscanner with a cylindrical phantom made of breast material, in which a smaller cylinder made of silicon was introduced......

Figure 4.4 Picture of the GATE platform, showing the source dislocation. 56

Figure 4.5 Cylindrical phantom in the simulation platform. 59

Figure 4.6 Diagram illustrating the position of the different objects inside the major cylindrical phantom. 59

Figure 4.7 Transversal slices of a female NCAT phantom torso (from inferior to superior slices). 60

Figure 4.8 Transversal slices of the breast phantom (from superior to inferior). 61

Figure 4.9 Coronal slices of the breast phantom (from anterior to posterior). 61

Figure 4.10 Sagittal slices of the breast phantom (from right to left). 61

Figure 4.11 The breast volume of NCAT phantom as it is presented in GATE. 62

Figure 4.12 Picture showing a mammographic acquisition in GATE. 63 
Figure 5.1 The $1 D$ projection at angle $\theta, y_{\theta}$ is the integral of the object distribution $x$. The distribution $f$ is the input that we want to estimate. 66

Figure 5.2 Different projections of the original object. 66

Figure 5.3 Image reconstruction using the inverse Radon transform taking an angle range of 50, 90, 180, 360 degrees, $a, b, c$ and d, respectively.

Figure 5.4 Diagram illustrating that the ray is attenuated only by the voxels that are crossed by it.

Figure 5.5 Diagram illustrating the intersection between a thin beam and a voxel on $2 D$.

Figure 5.6 Diagram illustrating the projections on the $X O Z$ and $Y O Z$ plans of the $3 D$ image showed in Figure 5.5.

Figure 5.7 Diagram presenting the chosen algorithms, FBP, ML-EM and ART, and theirs categories

Figure 5.8 Diagram illustrating the principle of the FBP algorithm. 76

Figure 5.9 Diagram illustrating the steps used to perform the ART algorithm.

Figure 5.10 Scheme illustrating the principle of the ML-EM algorithm. 79

Figure 6.1 Result obtained by the cylinder phantom. The figure shows the number of photons that reaches the detector (black is the null counting and white represents a detected photon).

Figure 6.2 Results of the simulation using the model described in Table 4.3. The 11 images are taken each 5 degrees performing an arc of 50 degrees.

Figure 6.3 Simulated data using the NCAT breast phantom. 11 projections were taken around an arc of 50 degrees each 5 degrees. 88

Figure 7.126 of the 50 slices of the volume estimated by the FBP reconstruction method. 95

Figure 7.2 Localization on the space of a region of interest selected, using crosshairs on the resultant volume. 96

Figure 7.3 Slices of the volume reconstructed by the ART algorithm. 99

Figure 7.4 Localization on the space of the mass, using crosshairs on the resultant volume. 
Figure 7.5 The 26 selected slices of the volume reconstructed by the ML-EM method.

Figure 7.6 Volume visualization on the transversal, sagittal and coronal plans. 104

Figure 7.7 Artifact spread function of the ellipsoid object for the three algorithms. ..106

Figure 7.8 Artifact spread function of the sphere (of $2 \mathrm{~mm}$ ) object for the three algorithms.

Figure 7.9 Artifact spread function of the cylinder object for the three algorithms....107

Figure 7.10 Slices 2, 11, 25, 32, 39 and 47 from a volume resultant from the back projection of a uniform image, using the system matrix constructed.... 


\section{List of Tables}

Table 1.1 Description of the plan of work

Table 2.1 Description of the stages of the tumour $[8,23]$ 18

Table 2.2 Description of the stages of the spread to lymph nodes [8] [23]. 18

Table 2.3 Description of the stages of the metastasis[8] [23] 18

Table 2.4 Correspondence between the stage grouping and the TNM system, and its relative survival rate at 5 years [28] [29].

Table 4.1 Correspondence between technical specifications of the mammography unit and the parameters introduced to perform the simulation. 52

Table 4.2 Characteristics of the compounds introduced in GATE to perform an acquisition 55

Table 4.3 Characteristics of the compounds introduced to perform the acquisition of images for reconstruction.

Table 4.4 Coordinates of the different structures inside the major cylinder.

Table 4.5 Characteristics of the compounds introduced in GATE to perform an acquisition with the used of the voxellized phantom, the NCAT phantom.

Table 7.1 Contrast-to-noise ratio of the three algorithms, calculated for the ellipsoid, sphere and cylinder regions. 105

Table 7.2 Comparison between the object visualization on its localization layer...... 105

Table 7.3 Runtime comparison between FBP, ART and ML-EM methods. 108 



\title{
Acronyms
}

\author{
ART Algebraic Reconstruction Technique \\ ASCII American Standard Code for Information Interchange \\ ASF Artifact Spread Function \\ BP Back Projection \\ CC Cranio-caudal mammographic view \\ CERN European laboratory for particle physics \\ CNR Contrast-to-Noise Ratio \\ DBT Digital Breast Tomosynthesis \\ e Euler's number \\ EGSnrc Electron Gamma Shower, National Research Council \\ EGS Electron Gamma Shower \\ EM expectation-maximization \\ $\mathrm{e}^{-} \quad$ electron \\ FBP Filtered Back Projection \\ FLUKA FLUktuierende KAskade \\ GATE Geant4 Applicatiin For Tomographic Emission \\ Geant4 GEometry ANd Tracking \\ GFB Gaussian Frequency Blending \\ h Planck's constant \\ HQR Half Quadratic Regularization \\ HU Hounsfield Unit \\ H Healthcare \\ INFN National Institute for Nuclear Physics \\ LM Latero-medial mammographic view \\ LMF Lexical Markup Framework
}




\begin{tabular}{|c|c|}
\hline MATLAB & Matrix Laboratory \\
\hline MCNP & Monte Carlo N-Particle \\
\hline $\mathrm{MHz}$ & Mega Hertz \\
\hline MITS & matrix inversion tomosynthesis \\
\hline ML & maximum-likelihood \\
\hline$M L$ & medio-lateral mammographic view \\
\hline MLO & medio-lateral oblique mammographic view \\
\hline ML-EM & maximum-likelihood expectation-maximization \\
\hline ML-convex & maximum-likelihood with convex algorithm \\
\hline MRI & Magnetic Resonance Imaging \\
\hline NCAT & NCAT NURBS-based Cardiac-Torso \\
\hline PET & Positron Emission Tomography \\
\hline PHITS & Particle and Heavy Ion Transport Code System \\
\hline$R \& D$ & Research and Development \\
\hline SAA & shift and add \\
\hline SART & simultaneous algebraic reconstruction technique \\
\hline SLAC & Stan-ford Linear Accelerator Centre \\
\hline STIR & Software for Tomographic Image Reconstruction \\
\hline TACT & tuned aperture computed tomography \\
\hline $\mathrm{T}, \mathrm{N}, \mathrm{M}$ & tumour, lymph nodes, metastasis \\
\hline $\mathrm{T} 1$ & Iongitudinal relaxation of the spins \\
\hline $\mathrm{T} 2$ & transversal relaxation of the spins \\
\hline Z & atomic number \\
\hline${ }^{18} \mathrm{~F}$ & Fluorine-18 \\
\hline 18F-FDG & 18F-fluorodeoxyglucose \\
\hline $2 \mathrm{D}$ & two dimensional \\
\hline $3 \mathrm{D}$ & three dimensional \\
\hline 4D & four dimensional \\
\hline
\end{tabular}





\section{Introduction}

\subsection{Scope}

Accordingly to the World Health Organization, cancer caused around $13 \%$ of deaths worldwide in 2004. The leading cause of death by cancer among women is breast cancer [1]. In 2008, the American Cancer Society estimated that 182,460 new cases of breast cancer arisen in that year and 40,480 women died by this cause, representing the leading cancer type in Female sex on United States [2].

In Portugal, cancer is among the three leading causes of death, with a progressive increase in the number of cases [3]. Breast cancer is the most common type among women and it is estimated that one in eleven women will be diagnosed breast cancer [4-6].

The incidence is higher in the developed and in developing countries than in the underdeveloped countries, which can be associated with the higher life expectancy in those countries [7]. Figure 1.1 shows the breast cancer incidence world-wide. 


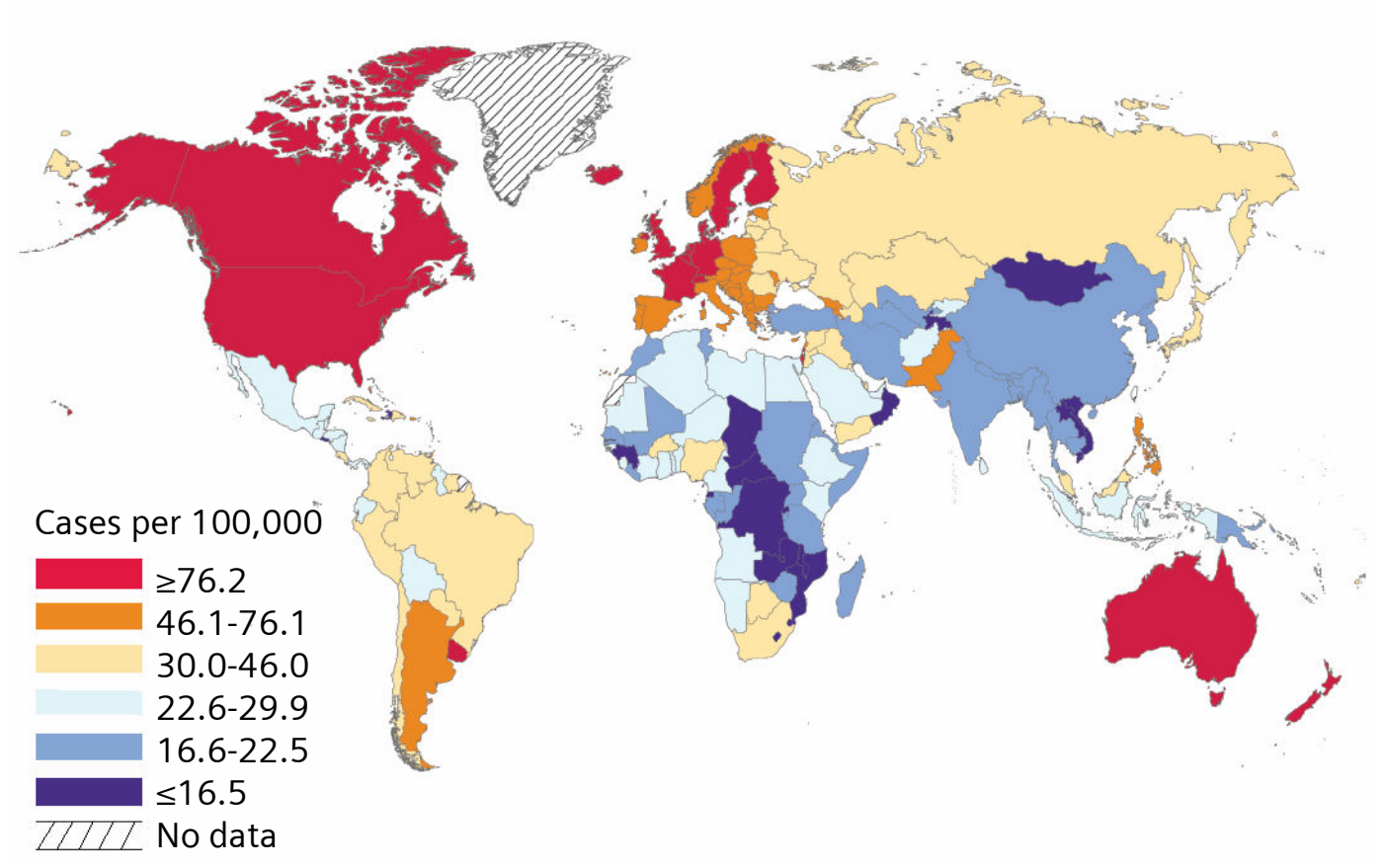

Figure 1.1 International variation in breast cancer incidence rates. [7]

The 5 year relative survival rate is very high when cancer is detected in an initial phase of the disease. In the case of breast cancer, the success of the treatments is around $100 \%$ on the earlier stages [8].

This high success rate of the treatments results of two major efforts on the fight against breast cancer:

- The awareness to the regular breast self-exam that plays a major role on early detection and the attentiveness to the early recognized of signs;

- The screening campaigns to detect cancer and pre-cancerous stages before the symptoms manifest [1].

The combination of the characteristics of breast cancer: high incidence, deadly disease asymptomatic at earlier stages and high survival rate at premature phase, makes screening and accurate diagnostic issues of an extreme interest. These outcomes the fight against breast cancer worthy of all the research done in the area and of the highest technology applied in the devices of breast imaging.

With the aim of reduce the damage caused by breast diseases, new image techniques have arisen, as the breast thermography or the breast tomosynthesis. Moreover, the current techniques are in constantly improving to make the diagnosis more accurate as 
possible. Thanks to these aspects, the mortality rate caused by breast diseases has decreased in the last years $[9,10]$.

It is generally accepted that mammography is the leader imaging technique of breast. It has been proved that reduces the mortality by breast cancer when it is used as screening practice $[9,11-14]$.

Mammography has a high sensitivity and specificity $(60.0 \%$ to $86.8 \%$ and $68.5 \%$ to $98.6 \%$, respectively) [15-18]. It is the gold standard for breast cancer screening and diagnostic, since it has this high performance at low cost. Nevertheless, as the 3D anatomical information is projected into a 2D image plan, some problems arise as overlapping tissue, loss of sensibility and specificity between tissues. This can mask a tumour or show a false-positive that will result on a recall. On the first case, if a tumour is not detected, this will delay the diagnostic and decrease the probability of a successful treatment, decreasing the survival rate and raising the costs of the future treatment. On the other hand, a false-positive diagnosis will cause anxiety to the woman, resulting on a recall and additional costs, when in general the findings are proved benign.

The emerging technique that may resolve the mammography gaps is the Digital Breast Tomosynthesis (DBT). This recent technology will allow the 3D reconstruction from mammograms, solving many problems. DBT:

- will increase sensibility

- will increase the specificity, allowing distinguish the malignant from the benign tissue;

- will allow the 3D visualization without overlapping tissues;

- will reduce recalls and its costs,

- will assess the shape, size and location of a lesion.

The tomosynthesis is a long time reported technique, but only in the last years its application on breast imaging was developed. Tomosynthesis is based on the principle of tomography, where several projections of the same object are taken from different angles, allowing the 3D reconstruction. However, in tomosynthesis the number of images taken is lower, beyond the angular range, that is more restricted than in the tomographic technique. 
Different types of tomosynthesis reconstruction methods have been explored. However, the algorithms are not totally developed neither optimized. The lower number of projections and the limited angle of taken views implemented on tomosynthesis make the $3 \mathrm{D}$ reconstruction an arduous work to improve the DBT accuracy, sensitivity and specificity.

\subsection{Presentation of the project}

This project emerges in order to compare different algorithms and optimize them to apply on Digital Breast Tomosynthesis. With this aim, simulated data will be used to perform and assess the tri-dimensional reconstruction.

As can be seen in Table 1.1, the first stage of the project is the learning and the familiarization with the tools that underpin the work. After that, it comes the mammograms simulation. A platform of simulation based on Monte Carlo methods (the Geant4 Application for Tomographic Emission - GATE) is used to represent a mammographic acquisition. To perform it, the system was created based on a mammography unit, where phantoms are placed.

Analytical phantoms and a realistic breast phantom were used. The breast phantom comes from NCAT (4D NURBS-based Cardiac-Torso), a well-known phantom widely used in simulations of exams of Nuclear Medicine that also can be used on transmission tomography.

The Monte Carlo simulations are an important tool to access the image reconstruction, since it is known exactly the simulated object to then compare with the reconstructed image.

Once acquired the simulated data, the reconstruction is performed on the MATLAB system. The algorithm development for the image reconstruction is implemented in MATLAB, which allows the computing and the results visualization.

The algorithms tested are the state-of-art image reconstruction algorithms.

In Table 1.1, the plan of work proposed in the beginning of this project is detailed. Each task is linked to a month and they are all arranged according to their temporal order. 
Table 1.1 Description of the plan of work.

\begin{tabular}{|c|c|c|}
\hline Month* & Plan of work: 3D medical image reconstruction & \\
\hline \multirow{4}{*}{1} & Meet the group Siemens and Siemens Healthcare Sector & \\
\hline & Basic training in breast Anatomy and Physiology; & \\
\hline & $\begin{array}{l}\text { Know the products, systems and solutions from Siemens: } \\
\text { Products of Mammography } \\
\text { Methods and Techniques for Acquisition } \\
\text { Digital Breast Tomosynthesis }\end{array}$ & \\
\hline & $\begin{array}{l}\text { Knowledge of the tools already at Siemens: } \\
\text { Applications for Conversion and Image Segmentation } \\
\text { Linux Operative System } \\
\text { Platform for Project Management R\&D Siemens. }\end{array}$ & \\
\hline \multirow[t]{2}{*}{2} & $\begin{array}{l}\text { Research and systematization: } \\
\text { Diagnostic Mammography. } \\
\text { Simulation and the Monte-Carlo Simulation Package } \\
\text { GATE (Geant4 Application for Tomographic Emission) } \\
\text { Algorithms for Reconstruction and Reconstruction } \\
\text { Package STIR (Software for Tomographic Image } \\
\text { Reconstruction) }\end{array}$ & \\
\hline & Writing and presentation of research conducted. & \\
\hline \multirow{6}{*}{3,4} & Familiarization with Development Environment $\mathrm{C}++$. & \\
\hline & $\begin{array}{l}\text { Installation and Release of GATE Simulations for } \\
\text { Benchmark }\end{array}$ & \\
\hline & Learning to use the tool to Voxellized Phantom GATE_v4 & \\
\hline & Learning of new functionality CTscanner of GATE_v4. & \\
\hline & Installation of STIR & \\
\hline & $\begin{array}{l}\text { Sample preparation for standard simulation (phantom } \\
\text { NCAT) for Mammography }\end{array}$ & \\
\hline \multirow[t]{2}{*}{$5,6,7$} & $\begin{array}{l}\text { Simulation data of Mammography / Digital Tomosynthesis } \\
\text { with the new functionality CTscanner of GATE_v4 }\end{array}$ & \\
\hline & $\begin{array}{l}\text { Adaptation of the Simulation Output (ASCII and ROOT) for } \\
\text { methods of reconstruction (sinograms, LMF). }\end{array}$ & \\
\hline
\end{tabular}




\begin{tabular}{|l|l|l|}
\hline Month* & Plan of work: 3D medical image reconstruction \\
\hline \multirow{8}{*}{$\begin{array}{l}\text { Implementation of FBP and iterative algorithms to } \\
\text { Tomosynthesis on MATLAB }\end{array}$} & \\
\hline & $\begin{array}{l}\text { Comparison of results with different algorithms with } \\
\text { simulated data and real data. }\end{array}$ & \\
\hline $\mathbf{9}$ & $\begin{array}{l}\text { Writing the Dissertation of Master } \\
\text { Preparation of the Conference Article (choose the most } \\
\text { appropriate) }\end{array}$ & \\
\hline & \begin{tabular}{l} 
Writing of Summary Report and Extended. \\
\hline
\end{tabular}
\end{tabular}

* The beginning of the project occurred on $21^{\text {st }}$ October.

Task performed within the deadline.

Task performed outside the deadline.

Not all of these tasks happened on the time proposed, since some of them took more time than the fixed period. This delay occurred for several motives:

- Time consuming adaptation to the Linux environment and to the use of the GATE platform and the NCAT phantom program.

- Difficulties on the familiarization process with the CTscanner device. This device is a new functionality available on GATE and there is few information about how to use it on GATE Users Guide.

- Complications with STIR installation. There were several problems on STIR compilation, probably linked to the Linux distribution used. The issues were overcome with the installation of another Linux distribution.

- Difficulty in completing the Monte Carlo simulations. The memory of the computer available for this project was too low to allow the complete simulation.

- The especial geometry of the studied case caused the alteration of the plan of work. The use of the STIR software to do the image reconstruction would be an arduous task given this geometry. The solution was to move for MATLAB and to write the whole algorithm. 
Concluding, the proposed plan was ambitious and challenging. The time for some tasks was underestimated in the initial plan and it had to be adapted and reviewed during the course with the supervisors.

\subsection{Technologies employed}

Diverse tools were needed to complete this work as proposed. The technologies described below were fundamental to perform the tasks succinctly explained in the following points.

\subsubsection{Geant4 Application for Tomographic Emission}

The GATE (Geant4 Application for Tomographic Emission) software is a platform for Monte Carlo simulations based on the Geant4 libraries. On the scope of the present work, this tool was used to simulate the Digital Breast Tomosynthesis exam, with the aim of obtaining images to reconstruct. GATE_v4.0.0 was the version installed.

\subsubsection{NCAT phantom}

4D NURBS-based Cardiac-Torso (NCAT) is a well-known phantom that plays realistically the anatomy and physiology of human body. On the scope of this project, just the breast volume was applied to complete the mammograms simulations on GATE.

\subsubsection{Milipeia}

Milipeia is a computer cluster installed in the University of Coimbra, in Portugal. It is used by several national and international groups of researchers. Under this project, Milipeia was an important device to complete the Monte Carlo simulations.

\subsubsection{MATLAB}

MATLAB (MATrix LABoratory) is a language for technical computing that was used to manipulate the images from projections and to resolve the problem of the $3 \mathrm{D}$ image reconstruction. The version used was MATLAB 6.5. 


\subsection{Contribution of the work}

The present work takes part of a major project aimed to provide an optimized solution for the image reconstruction on the recent device that is the Digital Breast Tomosynthesis.

To achieve this task a vast knowledge was acquired and it is expected that it will be an important tool to successfully reach the major goal: to allow the early and real diagnosis for the breast cancer, that is a worrying problem as it was reported on the presentation of the project.

For the particular case of Siemens Healthcare Portugal, all the research did on the scope of this project is a further step towards its assertion on the research and development of Siemens global milestones.

Furthermore, this work contributed to the enlargement of my knowledge about Monte Carlo simulations and image reconstruction and also about some tools to perform them. Apart whole the practice on research, the inclusion of this work on a company, allowed the acquisition of an organizational vision, the enlargement of the network and the incorporation on a corporative group. Diverse opportunities were given and they present a real asset to this work. As the participation on the World Congress 2009, the learning on diverse workshops and formations, the visit to a Service of Imaging, the opportunity to do social actions, and the chances of presenting this work to other research groups.

\subsection{Publications}

The work developed on Monte Carlo simulations during this project resulted on an oral presentation on the World Congress of Medical Physics and Biomedical Engineering, presented in Annex 1.

- Duarte, I.C., Caldeira, L., Soares, F., Silva, J.S., Janela, F., GATE Mammogram Simulation of NCAT Breast Phantom, in Medical Physics and Biomedical Engineering World Congress 2009. 2009: Munich.

A poster about this work was presented on the Medical Physics Workshop (Annex 2):

- Duarte, I.C., Caldeira, L., Soares, F., Silva, J.S., Janela, F., 3D Medical Image Reconstruction, in Medical Physics Workshop, Where is Portugal in the Medical Physics world?. 2009: Aveiro.

Further work is being prepared for submission in other conferences and possibly a journal paper. 


\subsection{Siemens S.A. presentation}

With 500 production centres in 50 countries and representation in 190 countries, Siemens is spread all over the world. In Portugal, Siemens S.A. encloses two factories, software research \& development centres (Lisbon and Porto) and has a significant representation all over the country through its partners and company headquarters. Since 2008, the company is organized in three major sectors: Industry, Energy and Healthcare [19].

The Healthcare Sector stands for innovative products and complete solutions, as well as service and consulting in healthcare industry. This Sector is organized in three divisions: Imaging \& IT, Workflow \& Solutions and Diagnostics [19].

The Imaging \& IT Division provides imaging systems for early diagnosis and intervention, as well as for a more effective prevention, namely Magnetic Resonance Imaging Systems, Computer Tomography Systems, Radiography and Angiography Systems, Positron Emission Tomography Systems, Single-Photon Emission Tomography Systems, Ultrasound Units, among others. These systems are networked with highperformance healthcare IT to optimize processes (such as hospital data systems like Soarian ${ }^{\circledR}$, image processing systems like Syngo ${ }^{\circledR}$, and knowledge-based technologies for diagnoses support) [19].

The Workflow \& Solutions Division provides complete solutions for fields such as cardiology and oncology and neurology. This Division offers solutions for, e.g. women's health (mammography), urology, surgery and audiology. It also provides turnkey solutions (including national health IT systems, complete solutions for healthcare providers), and consulting. In addition, Workflow \& Solutions is responsible for the Sector's service business and for managing customer relations [19].

The Diagnostics Division covers business with in-vitro diagnostics, including immune diagnostics and molecular analysis. The Division's solutions range from point-of-care applications to automation of large laboratories [19].

Thus, Siemens Healthcare Sector is the first fully integrated diagnosis company, providing a complete technological portfolio for the entire supply chain in healthcare Siemens IT Solutions and Services, leader in Information Technologies services, works as a transverse business unit [19].

In Portugal, Siemens S.A. Healthcare Sector is a market leader in the healthcare area, known for its competence and innovation skills in diagnostic and therapy systems, as 
well as information technologies and systems' integration. In recent years, Siemens S.A. Healthcare Sector has promoted the contact and cooperation with key partners in the areas of science and biomedical technology, namely Universities and Research Institutes, establishing a knowledge network and strategic partnerships and thus promoting innovation, research and development in healthcare [19].

Today, the Healthcare Sector's R\&D Group in Portugal is comprised by over 15 elements, working in strategic areas, such as Information Systems, Computational Imaging, Automatic Medical Imaging Analysis, Modelling and Decision Support Tools and Strategic Technology Evaluation. This work has already been demonstrated by one approved patent application, two filed invention disclosures and over ten scientific publications [19].

\subsection{Organization of the chapters}

The present report is divided in 8 chapters. The intention of this first chapter is to present the project and its contribution to research, to Siemens S.A. and to my personal training. The motivations to complete this work, the steps to perform it and a general view are described in this section.

The second chapter refers to breast imaging methods with a brief introduction about breast anatomy and physiology, breast cancer and the cancer screening and diagnostic. Thereafter, different technologies to perform the diagnostic of breast diseases are explained to give an idea about the possibilities to do it and justify the need to develop tomosynthesis for breast imaging.

The next chapter explains the basic principles of radiography and tomosynthesis and its application on mammography. The X-ray generation and its interaction with matter are detailed in this chapter. The adjustments that mammography must undergo, to allow the tomosynthesis technique, are also described here.

In chapter 4, the methodology used to perform the Monte Carlo (MC) simulations is detailed. A succinct description about what these methods are and how they will be used to perform simulated data as mammograms is presented. A state-of-art is given in conclusion for a brief idea of specific cases of mammography where MC methods have been used.

In the next chapter, a brief introduction about reconstruction methods and the state-ofart about DBT reconstruction techniques are done. Additionally, the algorithms that will 
be used for the reconstruction are described and the steps to perform them are detailed. The way how the algorithm performance will be analysed is explained in this chapter.

The chapter 6 presents the results of the Monte Carlo simulations. The acquired images are exhibited and the discussion about the results is performed in this chapter.

The results from the image reconstruction are displayed in chapter 7. Besides the results presentation, the algorithm performance is analysed in chapter 7 . The discussion about the results is done in the last sub-chapter.

The conclusions about this work are commented in chapter 8. 



\section{Imaging of the breast}

\subsection{Breast anatomy}

The breast is a glandular structure whose primordial function is the production of milk for nutrition of the baby. The breasts are mainly composed of glandular, fatty and fibrous tissues. The glandular tissue, producer of milk, is surrounded by fatty tissue that extends throughout the breast and determines its size. The fibrous components support the glandular tissue. The glandular tissue contains numerous lobules, milk producing glands, located at the end of the lobes [20].

During lactation, in response to hormonal signals, a muscle cells layer that involves the lobules (myoepithelial cells) is stimulated to press the lobules. Thereby, the lobules eject the milk to the ducts, which converge to the nipple carrying the milk. As the ducts get closer to the nipple and areola, there is a dilated part in which milk can be accumulated (lactiferous sinus). The sebaceous glands in the areola produce and secrete oily substance which lubricates the areola and the nipple [21]. 
The Cooper's ligaments are attached to the skin and the pectoralis muscles, keeping the breast suspended in the front side of the pectoralis muscles and chest wall (Figure 2.1) $[20,21]$.

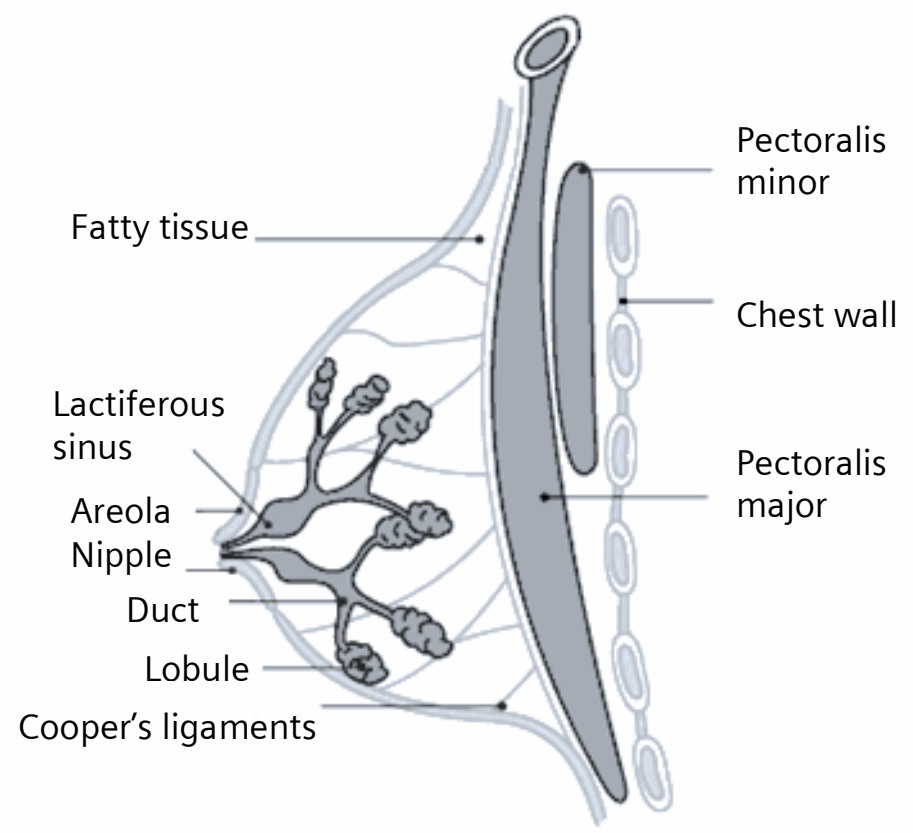

Figure 2.1 Structure of a normal breast, adapted from. [22]

\subsection{Breast cancer}

On normal tissue, the cells are in continuous growth and division. But this potential to replicate is restricted and it only occurs a finite number of times. If an abnormality arises, such as a mutation, the cell has a mechanism called apoptosis to program its own death.

A normal cell becomes a tumour cell, when a mutation occurs and it loses the ability to control its division rate. When that happens, the tumour cells grow and divide without being stimulated by growth factors. Moreover, they become insensitive to growth inhibitory signals and develop mutations that block apoptosis. In fact, most of the times, a tumour cell does not turn into a tumour. The last appears when the mechanism of apoptosis does not work [23].

As cancerous cells divide, they acquire high resistance and develop characteristics to assure their own survival. They develop the ability to induce the growth of new vessels (angiogenesis) to supply the essential nutrients for their rapid growth. They also 
develop others malignant characteristics, such the ability to evade the immune system, to mutate, to invade and metastasize [23].

The mutations in genetic code may be caused by some kind of internal or external stress. How exactly that occurs, it is not known yet. But it is known that certain factors are linked to increase breast cancer risk. The main risk is being a woman since breast cancer is 100 times more common among women than in men. Other risk factors are age, the use of birth control pills or hormone therapy, family and personal history of breast cancer, the race and ethnicity [2] .

Breast cancer arises when a tumour begins on the breast tissues. But even here, the cancer can be sourced in the different structures, such as ducts or lobules, and, moreover, the cancerous cell can have different characteristics. This differentiates the types of breast cancer. The most common breast cancer types are [24]:

- Ductal carcinoma in situ: are those that emanate from ductal cells but did not invade the surrounding breast tissue yet.

- Lobular carcinoma in situ: are those that emanate from lobular cells but did not invade the surrounding breast tissue.

- Invasive ductal carcinoma: the cancer cells arise from ductal cells, but invade the surrounding breast tissue and can travel to other parts of the body (metastasize).

- Invasive lobular carcinoma: the cancer cells form from lobular cells, invade the surrounding tissue and can metastasize

Others less common breast cancer types are [24]:

- Inflammatory breast cancer: it is an invasive carcinoma that spreads quickly to other parts of the body. It occurs when the lymph vessel is blocked by cancer cells.

- Medullary carcinoma: is an invasive carcinoma and forms a well defined boundary between the tumour tissue and normal tissue.

- Mucinous carcinoma: emanate from mucus-producing cells that invade the surrounding breast tissue.

- Paget's disease of the breast: it starts in ductal cells and spreads to the skin of the nipple and the areola.

- Metaplastic carcinoma: this carcinoma is a mixture of types of cancerous cells.

- Adenocarcinoma: are those that emanate from glandular tissue.

- Metastatic breast cancer: when the breast cancer spreads outside the breast and is found in other part of the body. 


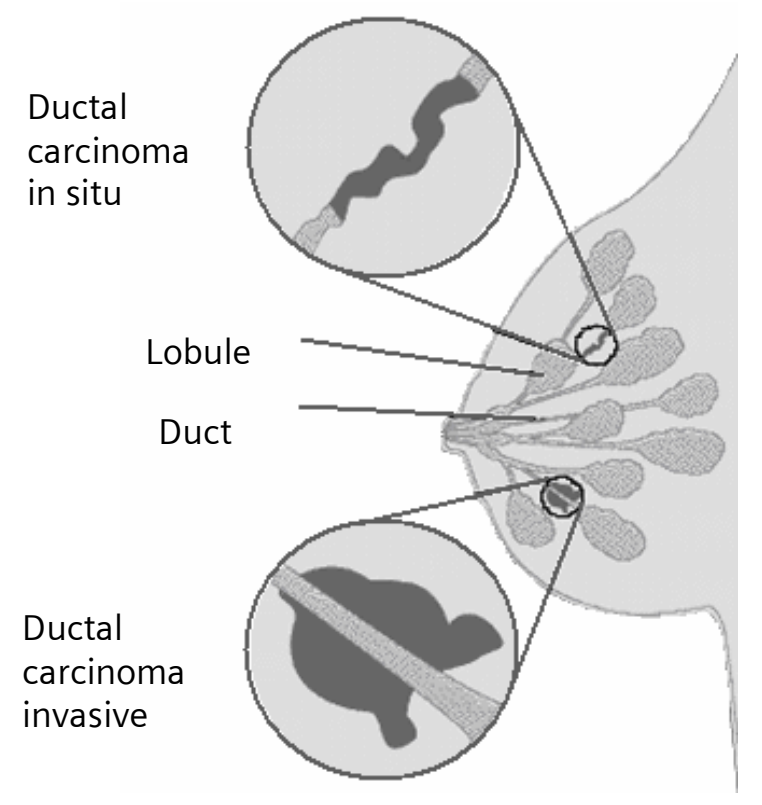

Figure 2.2 Picture of a breast, explaining the difference between in situ and invasive ductal carcinoma. In the last one, it is visible the invasion of the surrounding tissue by tumour ductal cells. Adapted from [25]

\subsubsection{Symptoms of breast cancer}

Many breast cancers have no obvious symptoms at all, especially in early stages. Moreover, many symptoms are common to other diseases of the breast.

Some of symptoms that can be related to breast cancer are [26]:

- Change in size, shape or feel of the breast;

- Pain, discharge or nipple retraction;

- Swelling or lump in the breast;

- Swelling in the lymph nodes;

- Scaly skin on nipple;

- Orange peel texture to breast;

- Unusual breast pain or discomfort;

On the later stages, other symptoms can appear, sometimes linked to metastases [26]:

- Bone pain; 
- Headaches, neurological pain or weakness;

- Shortness of breath;

- Drop in appetite.

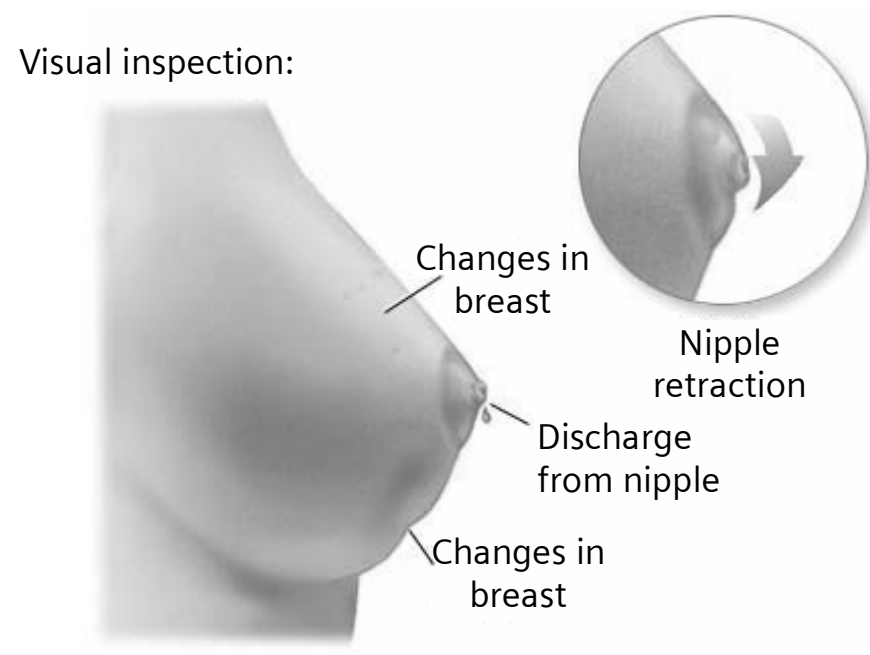

Figure 2.3 Appearance of some symptoms of breast cancer. Adapted from [27]

\subsubsection{Stages of breast cancer}

When the disease is found, it is extremely important to know in which stage the cancer is, in order to determine the prognosis and plan the treatment more deeply.

The classification of the tumour is based on its size, whether is invasive or non-invasive, degree to which the cancer has spread to the lymph nodes, and, metastasis existence [23].

A staging system is a standardized way for the oncologists to summarize information about the cancer characteristics and evolution. The most common system to describe is the TMN system $[8,23]$ :

- T stands for tumour (Table 2.1),

- N stands for spread to lymph nodes (Table 2.2),

- $M$ is for metastasis (Table 2.3). 
Table 2.1 Description of the stages of the tumour. $[8,23]$

\begin{tabular}{|l|l|}
\hline T & Primary tumour \\
\hline TX & Primary tumour cannot be assessed \\
\hline T0 & No evidence of primary tumour \\
\hline Tis & Carcinoma in situ \\
\hline T1 & Tumour is 2 cm or less across \\
\hline T2 & Tumour is more than 2 cm but not more than $5 \mathrm{~cm}$ across \\
\hline T3 & Tumour is more than 5 cm across \\
\hline T4 & Tumour of any size growing into the chest wall or skin \\
\hline
\end{tabular}

Table 2.2 Description of the stages of the spread to lymph nodes. [8, 23]

\begin{tabular}{|l|l|}
\hline N & Nearby lymph nodes \\
\hline N0 & Nearby lymph nodes cannot be assessed \\
\hline N1 & $\begin{array}{l}\text { Cancer has spread to } 1 \text { to } 3 \text { axillary lymph node(s), and/or tiny amounts of cancer are } \\
\text { found in internal mammary lymph nodes on sentinel lymph node biopsy }\end{array}$ \\
\hline N2 & $\begin{array}{l}\text { Cancer has spread to } 4 \text { to } 9 \text { axillary lymph nodes under the arm, or cancer has enlarged } \\
\text { the internal mammary lymph nodes }\end{array}$ \\
\hline N3 & $\begin{array}{r}\text { One of the following applies: } \\
-\quad \text { Cancer has spread to } 10 \text { or more axillary lymph nodes. } \\
-\quad \begin{array}{l}\text { Cancer has spread to the lymph nodes under the clavicle (collar bone). } \\
-\quad \text { Cancer has spread to the lymph nodes above the clavicle. }\end{array} \\
\text { - Cancer involves axillary lymph nodes and has enlarged the internal } \\
\text { mammary lymph nodes. } \\
\text { are found in internal mammary lymph nodes on sentinel lymph node biopsy. }\end{array}$ \\
\hline
\end{tabular}

Table 2.3 Description of the stages of the metastasis. [8, 23]

\begin{tabular}{|l|l|}
\hline M & Metastasis (M) \\
\hline MX & Presence of distant spread (metastasis) cannot be assessed \\
\hline M0 & No distant spread \\
\hline M1 & Spread to distant organs is present \\
\hline
\end{tabular}


The identification of the cancer stage may be done based on clinical breast exams, biopsy and certain imaging tests such as mammograms, chest $\mathrm{X}$-ray, bone scans, computed tomography scans, magnetic resonance imaging, ultrasound or positron emission tomography scans.

The 5 year relative survival rate according to stage grouping and its correspondence to the TNM system can be seen in Table 2.4 .

Table 2.4 Correspondence between the stage grouping and the TNM system, and its relative survival rate at 5 years. $[28,29]$

\begin{tabular}{|c|c|c|c|}
\hline Grade & \multicolumn{2}{|c|}{ TNM Correspondence } & survival rate \\
\hline Grade 0 & \multicolumn{2}{|c|}{ Tis NO MO } & $100 \%$ \\
\hline Grade I & \multicolumn{2}{|c|}{ T1 NO MO } & $100 \%$ \\
\hline \multirow{5}{*}{ Grade II } & \multirow{3}{*}{ Ila } & TO N1 Mo & \multirow{3}{*}{$92 \%$} \\
\hline & & T1 N1 Mo & \\
\hline & & T2 NO MO & \\
\hline & \multirow{2}{*}{$\mathrm{Ilb}$} & T2 N1 M0 & \multirow{2}{*}{$81 \%$} \\
\hline & & T3 NO MO & \\
\hline \multirow{6}{*}{ Grade III } & \multirow{4}{*}{ IIla } & TO N2 MO & \multirow{4}{*}{$67 \%$} \\
\hline & & T1 N2 M0 & \\
\hline & & T2 N2 MO & \\
\hline & & T3 N1, N2 M0 & \\
\hline & \multirow{2}{*}{ IIIb } & T4 any N MO & \multirow{2}{*}{$54 \%$} \\
\hline & & any T N3 MO & \\
\hline Grade IV & \multicolumn{2}{|c|}{ Any T any N M1 } & $20 \%$ \\
\hline
\end{tabular}

It is important to note that at the initial stages of breast cancer the survival rate is very high and knowing that breast cancer is generally asymptomatic at these stages, the screening and early diagnostic are fundamental to decrease the death by cancer. 


\subsection{Breast cancer screening}

The breast self-exams and the mammography play a major role in the early detection of breast cancer [23].

At age 20, every woman should begin breast self-exams and perform it monthly. The regular breast self-exam helps women become familiar with the look and feel of the breast and facilitates the detection of changes that may occur [30].

The best time to perform a breast self-exam is a few days after menstrual period has ended, as the breasts are not tender. If the woman is no longer having menstrual periods, she must continue do it monthly [30].

Examining the breasts is an important way to find a breast cancer early, but not every cancer can be found this way. Therefore, a clinical breast exam by a professional every three years is advised. After age 40, each woman should have a mammogram every year, in addition to the breast self-exam [23].

Mammography is a type of medical imaging technique that uses $X$-rays and is used as form of screening to image breasts that have no symptoms of breast cancer or observable breast abnormalities. The purpose of screening mammography is the early detection of small tumours, improving the chances for successful treatment. Typically, for screening mammography, two views from each breast are taken: one from above (cranial-caudal view) and other from an oblique view (medio-lateral oblique view), (Figure 2.4).

As previously stated, the screening mammogram is extremely important in woman's health and as the age is a risk factor, women after age 40 should get mammograms every one to two years and yearly after 50 .

It is estimated that approximately $85 \%$ of breast cancers may be detected by mammography, though the sensitivities can vary according to the radiologist experience [31].

\subsection{Current techniques for breast imaging}

When a signal or a symptom appears, it is necessary to find the cause, whether it is due to cancer or to some other less serious disease. 
Palpation is the first step. Carefully feeling a lump, a physician can get information about its size, its texture and whether it moves easily or not.

Usually, the doctor needs an additional test; he may order a mammogram or another imaging procedure

\subsubsection{Diagnostic mammography}

Mammography is the most frequently form of medical imaging used in the early detection and diagnosis of breast diseases [32]. It is the gold standard for breast cancer screening and early detection [33].

The diagnostic mammography differs little from screening mammography. It may involve more views and it is done when there is something suspicious. In addition, it can focus a specific area of the breast where the signal was detected. The most common mammographic views are illustrated in Figure 2.4.
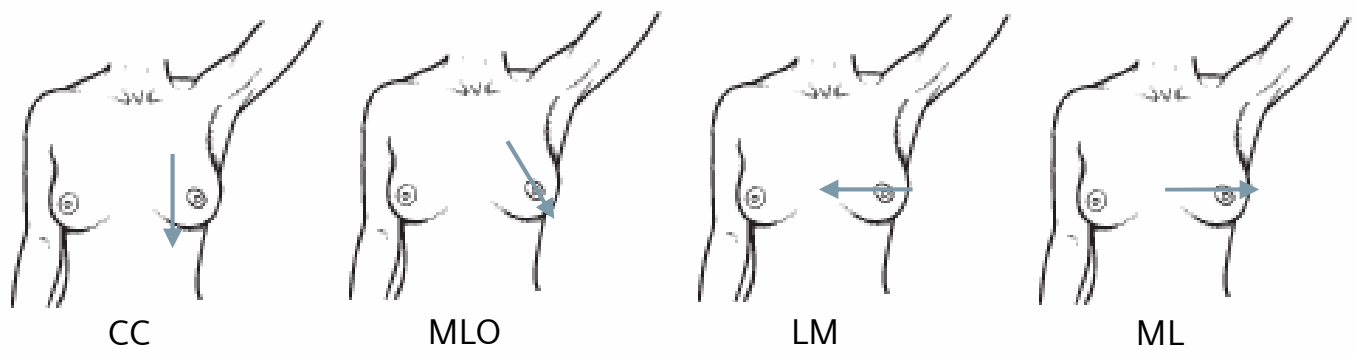

Figure 2.4 The most common mammographic views: cranio-caudal (CC) mammographic view, medio-lateral oblique (MLO) mammographic view, latero medial (LM) mammographic view, medio-lateral (ML) mammographic view.

Mammography is a low dose X-ray exam of the breasts which allows evaluating a breast abnormality detected. It is a non-invasive technique, but involves exposing a part of the body to an ionizing radiation. In mammography each breast is compressed and is crossed by an X-ray beam (Figure 2.5). The resulting beam reaches a film or an electronic detector (film or digital mammography, respectively). 


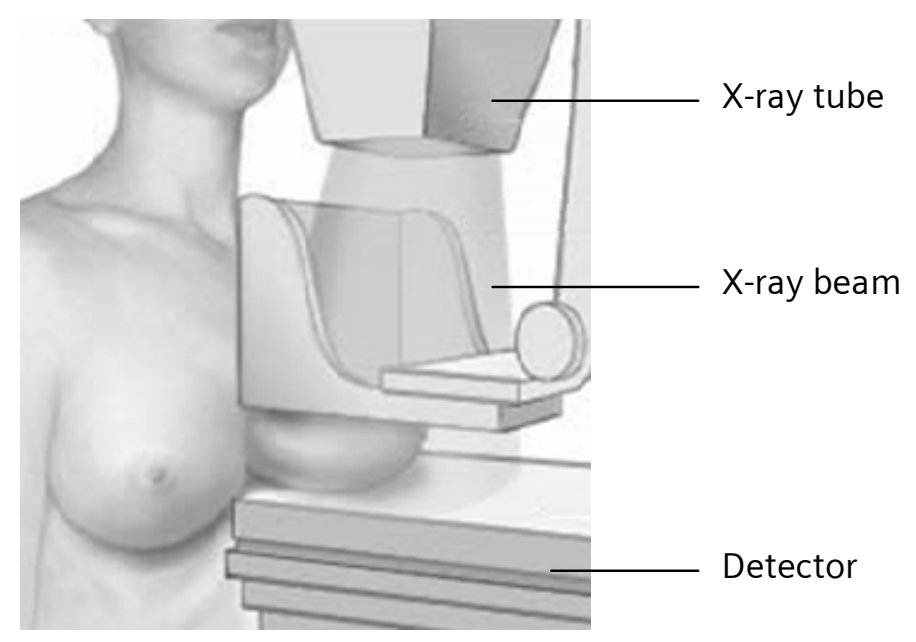

Figure 2.5 Representation of a mammographic acquisition. Adapted from [27]

The conventional mammography has been used since the 1960s. Here, the image is created directly on a film. In spite of it does not allow significant alterations, the diagnostic accuracy of film does not fall short from the digital for women over 50 years or with fatty breasts [34].

In digital mammography the X-ray is recorded digitally in a computer, facilitating its storage and transmission. The images are displayed on a computer monitor and a variety of tools can be used to manipulate them, facilitating the interpretation of the picture by the radiologist [35]. The diagnostic accuracy of digital mammography performs better for women under 50 years with dense breasts. A digital mammogram is displayed in Figure 2.6.

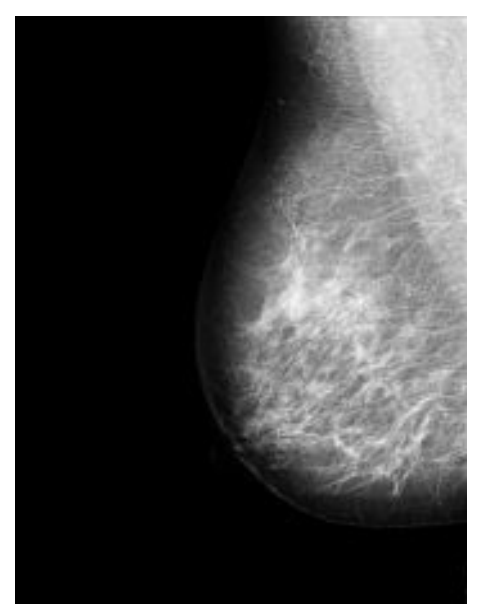

Figure 2.6 Digital mammogram. [36] 
Digital mammography provides some advantages over conventional mammography [35]:

- The use of lower radiation doses;

- The ability to manipulate images;

- The exposure variation does not interfere in the image;

- Reduction of exam times;

- Facility on storage and transmission of the images.

\subsubsection{Ultrasonography}

Ultrasonography is an imaging technique in which images are obtained from highfrequency sound waves, in the frequency range of $1-20 \mathrm{MHz}$. As the wave propagates through the structures of the body, it can be reflected, refracted, scattered or absorbed. The waves are emitted through the tissues by a transducer and a part of them are reflected at the tissues interfaces. The reflection of the ultrasound is received by the same transducer and is used to show the structure and movement of the body's internal organs, as well as blood flow [37]. Figure 2.7 is an example of the resultant image obtained using ultrasounds.

Ultrasound imaging is a non-invasive technique and does not involve the use of ionizing radiation. The images are used to evaluate lumps and may show whether a lump is a solid tumour or a fluid-filled cysts. Therefore, it can be a complementary exam to mammography, evidencing what is difficult to see on a mammogram [35].

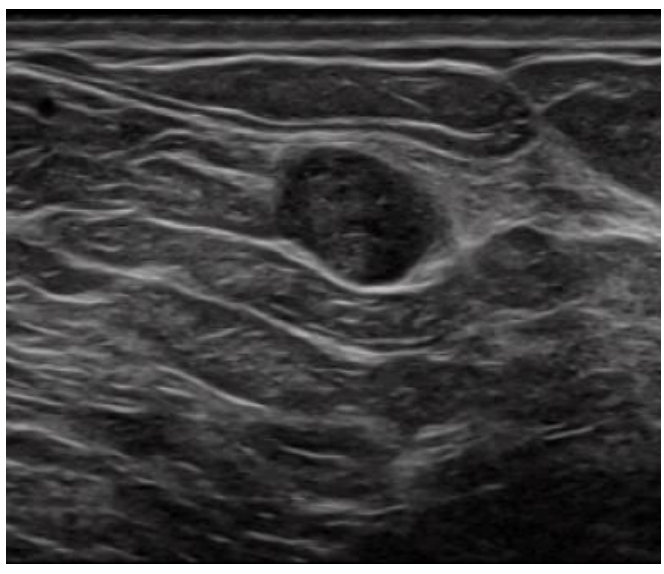

Figure 2.7 Ultrasound image of breast evidencing a fibroadenoma. [36] 


\subsubsection{Magnetic Resonance Imaging}

The magnetic resonance imaging (MRI) is based on the principal that the behaviour of the atomic nuclei is influenced by the application of external magnetic and radio frequency fields. Magnetic resonance uses magnetic fields and radiofrequency waves to create an image based on the magnetic property of the protons, called spin. The spins have random orientations, when they are not exposed to a magnetic field. But, when the protons are exposed to the effects of a strong magnetic field (commonly 1.5 T), their spins move on the same direction, staying parallel or anti-parallel to the field [37]. A radiofrequency wave is used to turn this magnetization to a transversal axle. After that, the spins return to their original stage of equilibrium and the duration of this movement is measure. The duration of longitudinal relaxation (T1) and transversal relaxation (T2) differ according the molecular properties of tissues. The image of magnetic resonance is based on $\mathrm{T} 1$ and $\mathrm{T} 2$ parameters and/or on the density of protons of the tissues (Figure 2.8) [37].

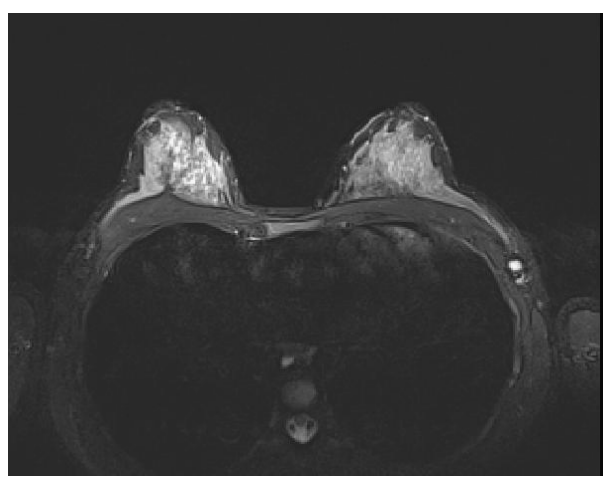

Figure 2.8 Resonance magnetic image of the breasts. [36]

MRI is a non-invasive medical test and does not use ionizing radiation. The magnetic resonance has been shown to be a sensitive and effective method of diagnosis of breast abnormalities. The basis of this high sensitivity is the tumour angiogenesis, for the reason that the angiogenesis increases capillary density and causes an abnormal permeability that facilitates a rapid spread of the injected contrast [38].

The magnetic resonance is especially used in young women, in women with dense breast tissue and in women with breast implants. It has been particularly effective for detection of very small tumours not detected through other tests. Magnetic resonance is also performed to assess multiple tumour locations, distinguish between scar tissue and recurrent tumours, and assess the effect of chemotherapy and staging breast cancer [38]. 


\subsection{Others techniques for imaging of the breast}

\subsubsection{Contrast-enhanced mammography}

This emerging technique, based on common mammography, utilizes iodinated contrast agents, with high X-ray attenuating, to detect some signs of angiogenesis. The contrast agent is administered through an injection and concentrates in the blood system, showing also the angiogenesis that accompanies the tumour growth [39].

There are two methods to performing that [39]:

- Dual energy contrast-enhanced digital subtraction mammography: two digital mammograms, one of low-energy and one of high-energy, are taken and subtracted one to other. The resulting image increases the visibility of blood vessels.

- Temporal contrast-enhanced digital subtraction mammography: two digital mammograms are taken, before and after contrast administration, and then subtracted.

\subsubsection{Breast scintigraphy}

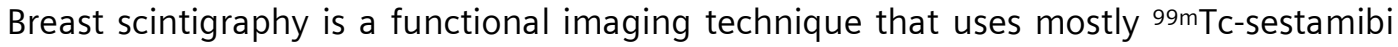
and $99 \mathrm{~m} T \mathrm{c}$-tetrofosmin, emitters of gamma-rays, to produce an image using a standard scintillation camera (Figure 2.9) $[40,41]$. Scintigraphy is mostly applied to evaluate breast cancer in patients in whom mammography is nondiagnostic, equivocal or difficult to interpret; to assist in identifying multifocal carcinomas in patients with tissue diagnosis of breast cancer, and may be useful in the evaluation of the effectiveness of neoadjuvant chemotherapy for breast cancer $[40,41]$.

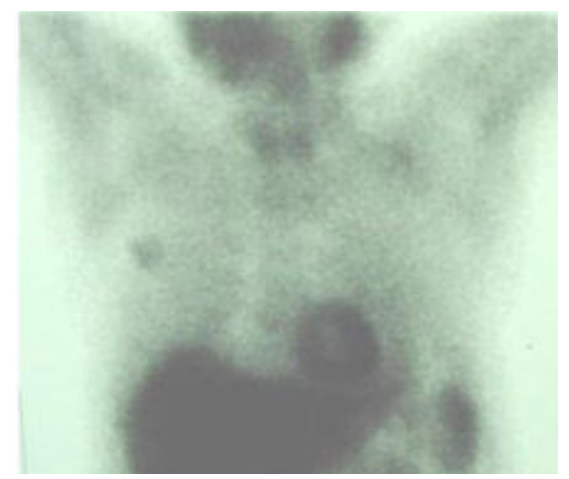

Figure 2.9 Scintigram of breast showing an intense soft tissue uptake in a carcinoma of the right breast. [42] 
Scintigraphy is a useful complement to mammography, as it is not affected by changes in breast morphology or by anatomical changes following the chemotherapy and radiotherapy. Moreover, it has a high sensitivity (except in smaller lesions). Thus, it can avoid unnecessary biopsies and may identify unexpected sites of breast cancer [40].

Breast scintigraphy is an ineffective exam to detect small breast tumours $(<1 \mathrm{~cm})$ and it has an high rate of false-positive cases [13].

\subsubsection{Breast Thermography}

Breast thermography is based on the principle that the high metabolism and angiogenesis associated to the cancer can increase the temperatures of the breast. A difference in temperatures between the tumour and the neighbouring tissues is expected. These differences are in order of $2^{\circ} \mathrm{C}$ [37].

An ultra-sensitive infrared camera and sophisticated computers are used to detect and produce diagnostic images according with the temperatures and vascular changes (Figure 2.10).

Breast thermography is a non-invasive technique, without contrast administration or exposure to ionizing radiation.

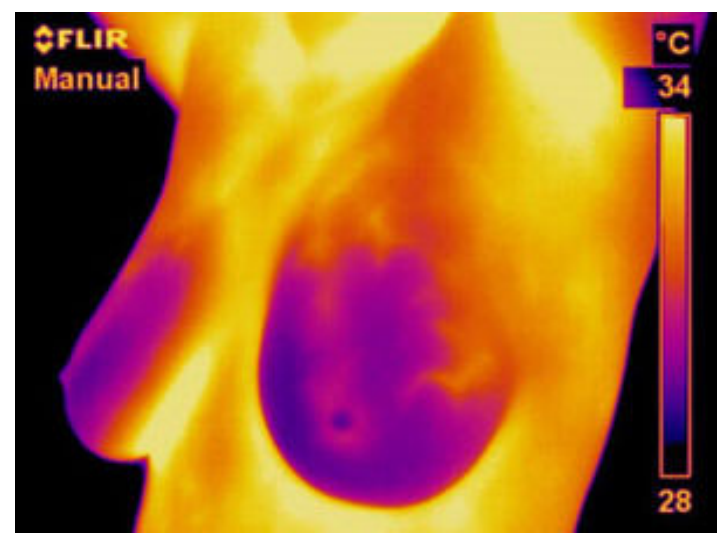

Figure 2.10 Image of corporal temperature acquired by thermography. [43]

The thermographic technique is known for long time, but its application in breast imaging was no longer used due its limitations. However, with the recent improvements of this technique, the interest in this application has increased.

The thermography can evidence breast cancer; nonetheless, it has limited success in the detection of early breast cancer and other breast diseases [37]. 


\subsubsection{PET scan}

Positron emission tomography is a functional imaging technique that can be used on breast cancer detection. Generally, it uses ${ }^{18} \mathrm{~F}$-fluorodeoxyglucose ( $\left.{ }^{18} \mathrm{~F}-\mathrm{FDG}\right)$, a modified and radioactive molecule of glucose, which has a higher uptake by cancer cells than normal cells. That is because the cancer cells have a very fast metabolism for which they have a high glucose uptake comparing with normal cells. Thus, the radioactive molecule will locate substantially in the area of the tumour, producing a functional image using a PET scanner that detects the radiation (Figure 2.11) [13].

The larger and more aggressive the tumour is, more accurate the PET scan results. It can be helpful in evaluating whether a lump is or not malignant, to detect distant metastasis or to evaluate the response to therapy.

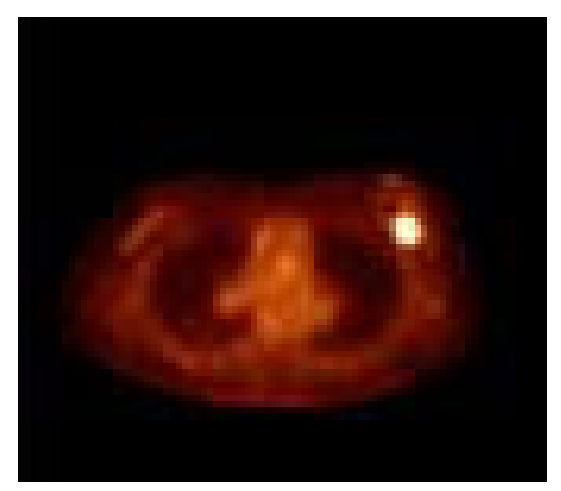

Figure 2.11 PET image evidencing a region of high uptake. [44]

\subsubsection{Positron Emission Mammography}

Positron emission mammography (PEM) is a breast dedicated imaging procedure that is still on an experimental phase. It is a functional imaging technique, which produces images based on the FDG uptake by the tissues. The applied doses and the exam duration are lower than in the PET exam [13]. This new technique brings advantages compared to the whole-body PET, since it has higher sensitivity and spatial resolution [45].

\subsubsection{Near infrared optical imaging}

In this method, near infrared light penetrates through the breast tissue and its absorption at several frequencies is measured. The image produced can show areas of angiogenesis and with a high metabolism. It is a non-invasive technique, without exposure to ionizing radiation, but it still has a very poor resolution [46]. 


\subsubsection{Bioelectric imaging}

This method is based on the principle that normal and tumour tissues have different electrical properties. An electrode array is placed on breast surface and introduces a low level of electricity into the body. The resulting electric field is measured by sensors to create a real-time electrical image of the breast [46].

\subsubsection{Digital Breast Tomosynthesis}

In Digital Breast Tomosynthesis (DBT), the breast is positioned as in conventional mammography with a soft pressure just to keep the breast in the same position during the exam. As it can be seen in Figure 2.11, an X-ray tube moves in an arc around the breast and multiple X-ray images are taken from different angles (about 11 projections are taken). These images are processed in a computer, composing highly focused tridimensional images throughout the breast. The radiation dose applied in DBT is similar to screening mammography $[46,47]$.

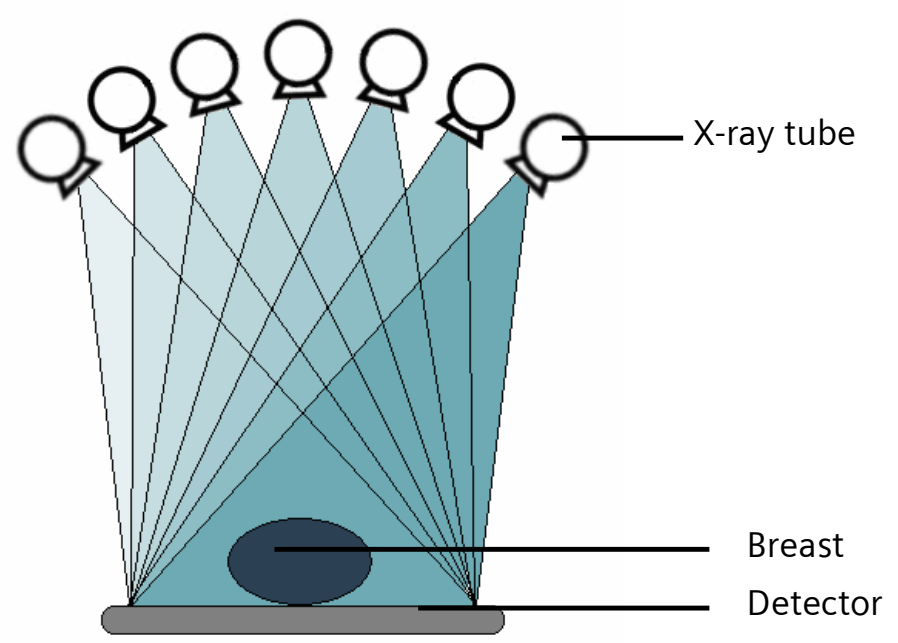

Figure 2.12 Schema of the acquisition of images in DBT, illustrating the movements of the $\mathrm{X}$-ray tube around the breast.

The DBT can bring several benefits, such as[46, 47]:

- reduce the compression of breast;

- improve the image of dense breasts;

- facilitates the analysis of breasts with implants;

- increase cancer detection rates;

- reduce recall rates.

The DBT may be applied for screening, diagnostic and biopsies. 


\subsection{Biopsy}

It is not always possible to evaluate whether a lesion is or not cancerous by imaging procedures. If so, it is necessary to collect some cells from the suspicious area, biopsy, and send them to a laboratory for a pathological analysis (Figure 2.13). This is the definitive way to determine the diagnosis [32].

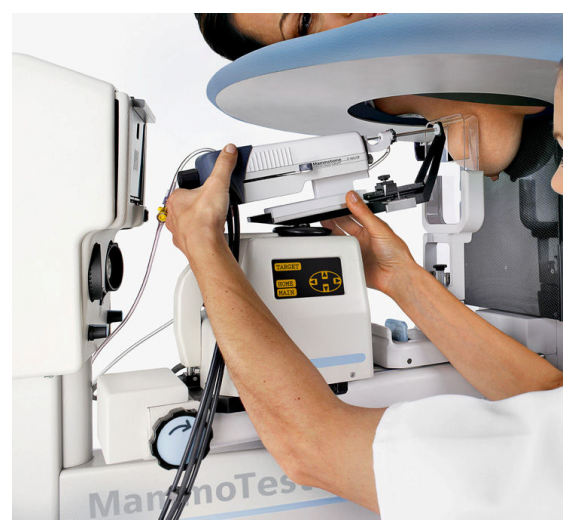

Figure 2.13 Picture showing a biopsy for breast. [36]

The collect of a tissue sample can be done by several methods. The choice by one of them depends on the size, location and other characteristics of the breast lesion. The different methods include [21, 30]:

- Fine-needle aspiration biopsy: is the most common type used to evaluate a lump. It uses a very fine needle and a syringe that can collect a sample of cells or fluid from the lump;

- Core needle biopsy: it is used a hollow needle, thicker than the used in fineneedle aspiration, to remove the tissue sample. Several samples can be collected, involving several insertions. This type may be used when the breast lump is palpable;

- Stereotactic, MRI-guided and ultrasound-guided core needle biopsy: is a technique equal to the previous, but is performed under image-guidance using stereotactic mammography, MRI or ultrasound (respectively);

- Surgical biopsy: it may remove part of the breast lesion (incisional biopsy) or it can attempt to completely remove the lesion (excisional biopsy);

- Vacuum-assisted biopsy: is a procedure to collect of multiple tissue samples at once, without involve several insertions. 



\section{Radiography}

\subsection{X-rays}

X-rays are a type of electromagnetic energy that is not visible to humans. As it can be seen in Figure 3.1, they have a wavelength in the range of $10^{-11}$ to $10^{-8}$ meters (that corresponds to frequencies in the range of $30 \times 10^{15}$ to $30 \times 10^{18} \mathrm{~Hz}$ ) and energies in the range of $120 \mathrm{eV}$ to $120 \mathrm{keV}$. The photon energy is proportional to frequency and it is given by equation 3.1. Where $v$ is the radiatiin Frequency and $\mathrm{h}$ is the Planck's constant and it is equal to $4.13 \times 10^{-18} \mathrm{keV} . \mathrm{Hz}[48]$.

$$
\mathrm{E}=\mathrm{h} v
$$

This type of electromagnetic radiation is used in medicine to produce radiographic images, an important tool in the diagnosis of diverse types of diseases. 


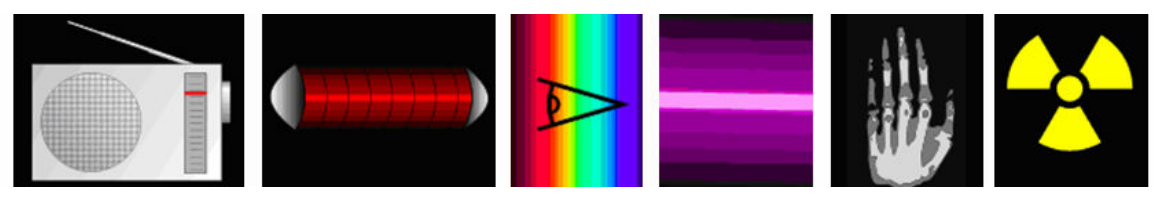

Wavelength $(\mathrm{m})$ :

Radio Microwave Infrared Visible Ultraviolet X-ray Gamma ray

$\mathrm{C}_{10^{3}}$

Frequency $(\mathrm{Hz})$ :

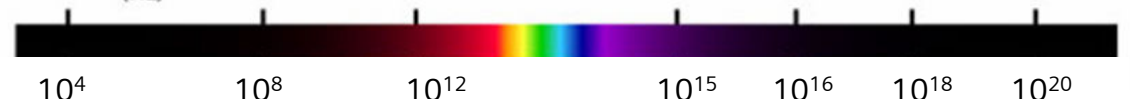

Figure 3.1 Electromagnetic spectrum, illustrating the wavelength and the wave frequency. Adapted from [49]

The X-ray beam is produced on an X-ray tube, a particular light bulb specially made to produce X-rays (Figure 3.2). This X-ray tube, a glass tube coated by oil and lead, is kept in vacuum, to prevent the interaction between the radiation and air. There are two electrodes, the cathode and the anode. The cathode is a coiled filament made of tungsten and the anode is the target, generally also made of tungsten. When a current is passed trough the filament, its electrical resistance causes the filament to heat up and discharge electrons by a process called thermionic emission. Between the cathode (negative electrode) and the anode (positive electrode) a voltage is applied and the cloud of electrons will flow towards anode $[13,48,50]$.

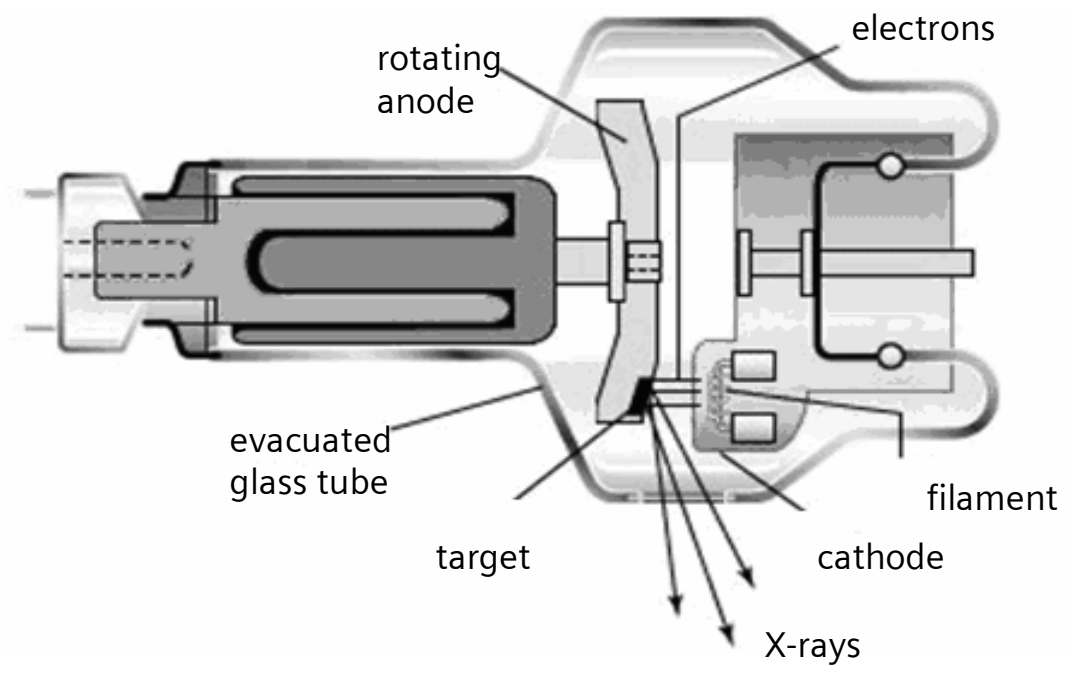

Figure 3.2 X-ray tube and its compounds. Adapted from [51] 
The electrons reach the target with a high kinetic energy and transfer it by interactions with the orbital electrons or with the nuclei of target atoms. The kinetic energy is converted mostly into thermal energy and only about $1 \%$ is converted into electromagnetic energy in the form of X-rays. Characteristic X-radiation can be produce by two forms [52]:

- Bremsstrahlung radiation, if the electron interacts with the nuclei of target atoms (Figure $3.3 \mathrm{a}$ );

- K-shell radiation, if the electron interacts with orbital electrons of target atoms (Characteristic X-rays) (Figure $3.3 \mathrm{~b}$ ).

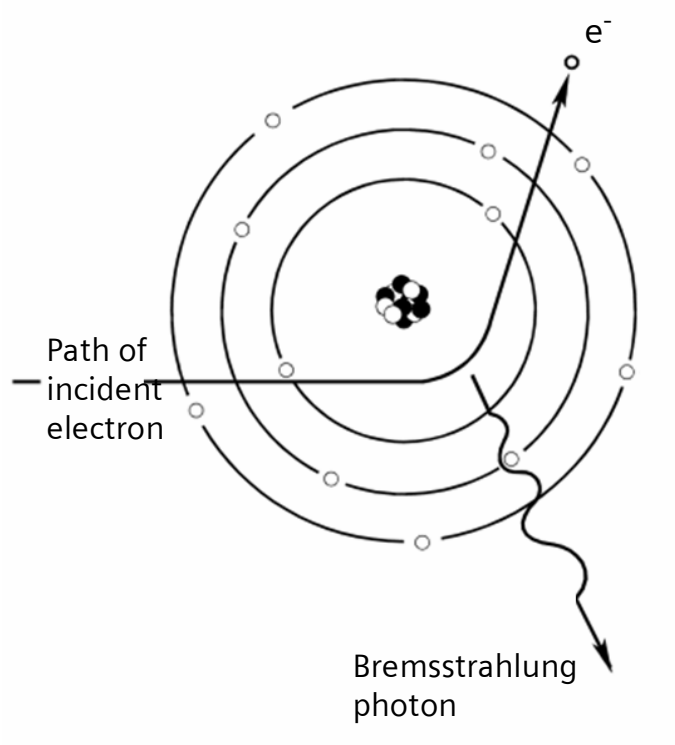

a)

\section{Ejection of orbital electrons}

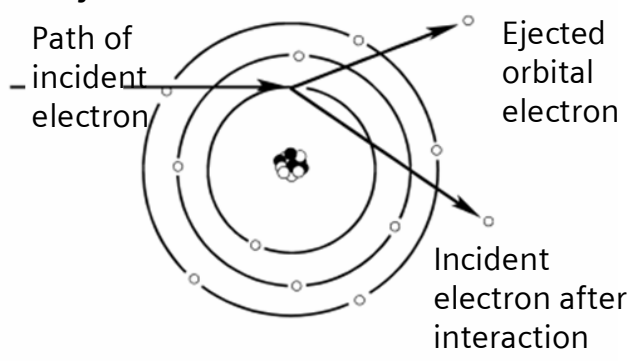

Emission of characteristic photons

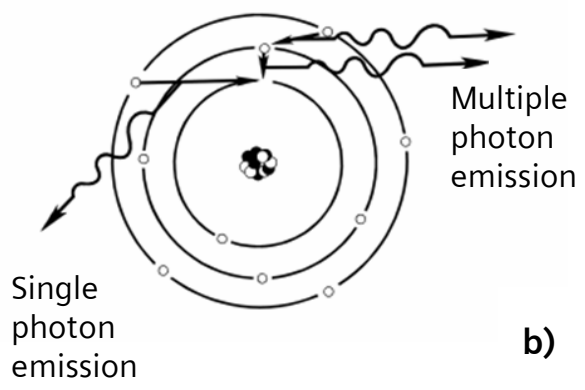

Figure 3.3 Generation of Bremsstrahlung radiation a) Generation of characteristic radiation b). [52]

When an incident electron penetrates the electron cloud, it is attracted to the nucleus. But its velocity is such that the electron does not stop and only is reduced its kinetic energy and deflected its trajectory. The lost energy is emitted as a Bremsstrahlung photon (X-ray) [52].

If the kinetic energy of incident electron is sufficient, it may ionize the atom by the removal of an inner-shell electron of the electron cloud. The hole created is occupied by an outer-shell electron and the excess energy is emitted as a characteristic photon [52]. 
The characteristic X-rays have discrete values of energy (Figure 3.4) and these values depend of the differences between electron binding energies, and this is a feature of each element. In the case of X-ray tubes used in medicine, the chosen target is a disc in rotation generally made of tungsten. This element has a high melting point $\left(3422^{\circ} \mathrm{C}\right)$, important given the inefficiency of the process of production of $X$-rays; and a high atomic number (74), important to increase the probability of interaction between the electrons and the target.

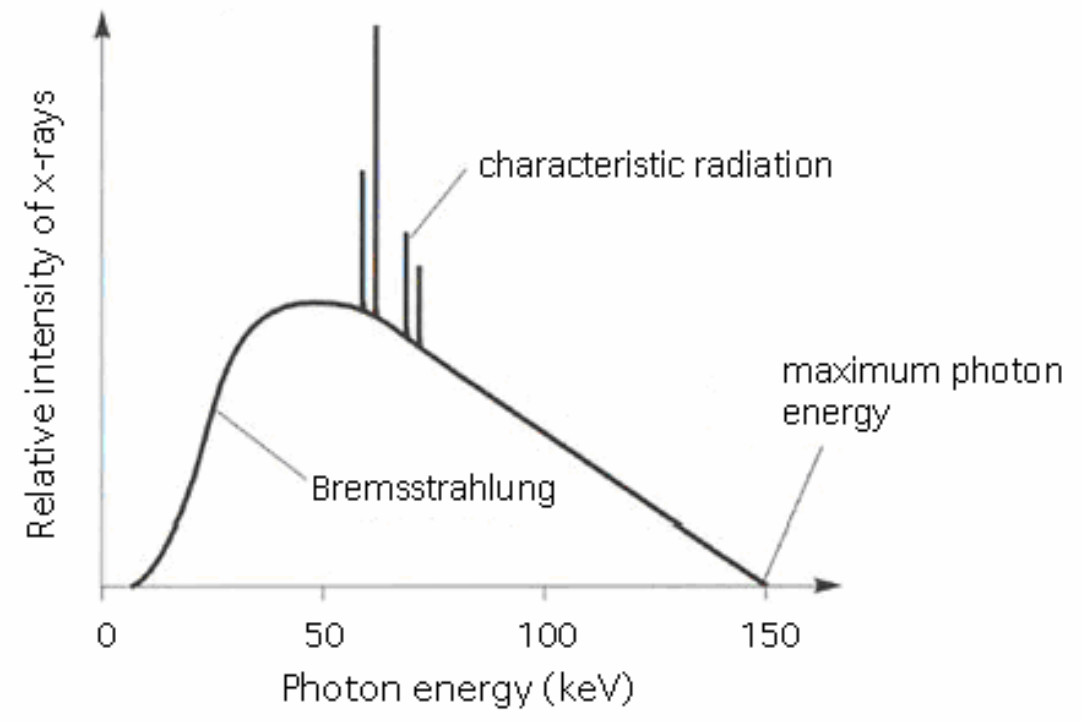

Figure 3.4 X-ray spectrum produced by a Tungsten target. Adapted from [53]

In radiology, the energy of the X-rays is generally measured in thousands electrons volts. An electron volt is the energy acquired by an electron as it traverses a voltage of one volt in vacuum. A higher voltage can be applied to increase the photon energy. This voltage, that provides the potential to accelerate the electrons, is pulsating. As result, the photons have different energies on a range. The increase of the voltage enlarges the velocity with which the electrons move from the cathode to the anode and consequently increases the penetrating power of the resulting X-rays [54, 55].

The amperage is used to control the temperature of the cathode and thus the intensity of the X-ray beam. Since the hotter the cathode, the more electrons are ejected towards the anode $[54,55]$.

The created X-ray beam emanate from focal spot which has to be a small area to prevent unsharpness (Figure 3.5). The unsharpness results from the penumbra of the object on the produced image. 

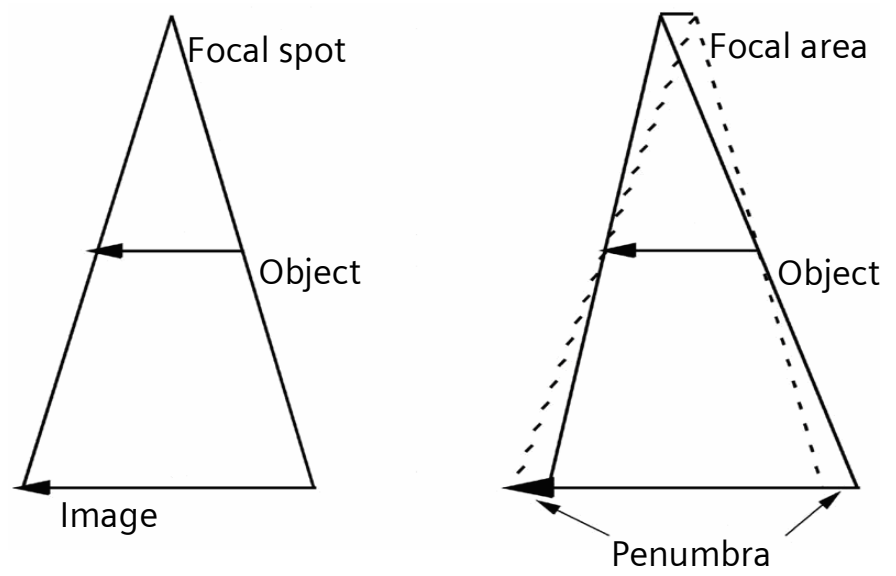

Figure 3.5 Illustration showing that the penumbra on the image has its origin on the increase of focal area. Adapted from [56]

\subsection{Interaction between $\mathrm{X}$-ray and tissues}

The photons in the X-ray beam penetrate the body and interact with the atoms of the tissues. As result of these interactions, the photon may be scattered or absorbed, more or less, depending of its energy and the properties of the tissues.

As illustrated in Figure 3.6, there are three major forms of interaction between X-rays (in the range of medical imaging energies) and matter:

- Compton Effect: the incident photon interacts with an outer-shell electron, ejecting it and ionizing the atom. The incident photon changes its direction and loses energy.

- Photoelectric Effect: the energy of the incident photon is totally absorbed by an electron of the cloud which is removed from the atom.

- Rayleigh scattering: the incident photon excites an electron and this releases the excess of energy as a photon. The scattered photon has the same energy as the incident photon, but has a different direction [57]. 
Mass Attenuation Coefficients for Soft Tissues

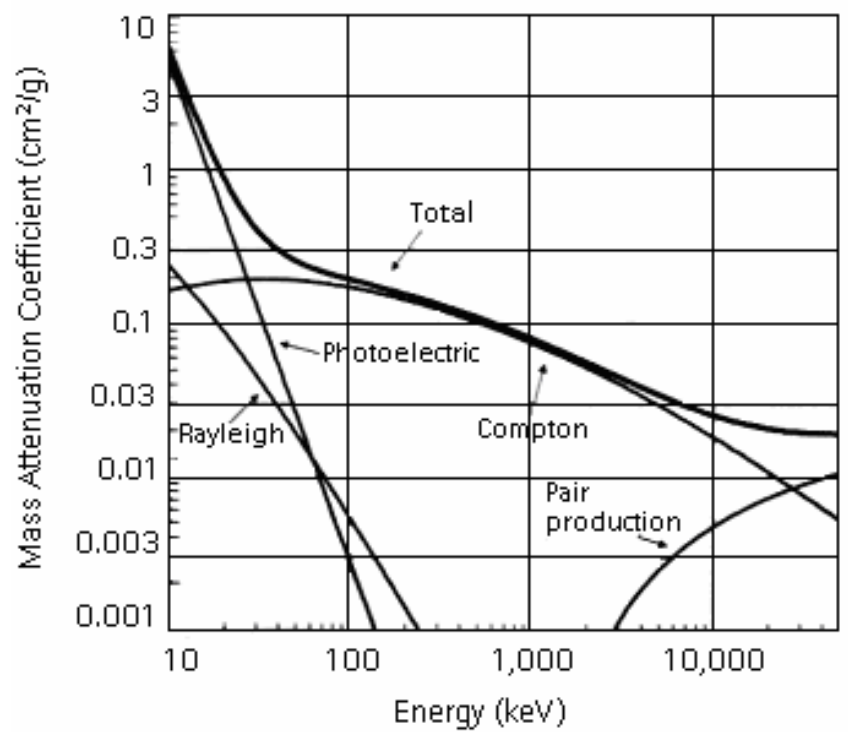

Figure 3.6 Graphic of total mass attenuation coefficients for soft tissues, resulting from the sum of the diverse forms of interaction between radiation and soft tissues. Adapted from [58]

The linear attenuation coefficient matches the fraction of X-rays that is absorbed or scattered per unit of thickness of the tissue, by the different types of interaction. Basically, this value describes the probability of a photon being scattered or absorbed by the tissue and is influenced by the atomic number, $Z$, of the material and its density and by the photons energy, E. This probability is higher, the greater the density of tissue. The linear attenuation coefficient decreases with the increasing photons energy [48, 59].

The variation of the intensity of the X-ray beam, $\mathrm{dl}$, at some distance on the tissue is given by equation 3.2, where $\mathrm{I}$ is the intensity, $\mathrm{n}$ the number of atoms $/ \mathrm{cm}^{3}, \sigma$ a constant of probability of the photon being scattered or absorbed and $\mathrm{dx}$ the thickness of the tissue crossed.

$$
d l(x)=-l(x) \cdot n \cdot \sigma \cdot d x
$$

The integrated form of equation 3.2 is equation 3.3, that gives us the transmitted intensity, I. Here e is the Euler's number and lo is the incident intensity [60]. 


$$
\mathrm{I}=\mathrm{I}_{0} \mathrm{e}^{-\mathrm{n} \sigma \mathrm{x}}
$$

The linear attenuation coefficient, $\mu$, is property of the material and is given by the result of the constant of probability of the photon being scattered or absorbed by the number of atoms $/ \mathrm{cm}^{3}$. As result comes equation 3.4, known by the Beer-Lambert law:

$$
\mathrm{I}=\mathrm{I}_{0} \mathrm{e}^{-\mu \mathrm{x}}
$$

The total attenuation of the X-ray beam is the result of the various attenuations caused by each form of interaction between photons and matter. Accordingly, the total linear attenuation coefficient, $\mu_{\text {tot }}$, is determined by equation 3.5. Where $\mu_{\mathrm{pe}}, \mu_{\mathrm{coh}}, \mu_{\text {incoh }}$ are the photoelectric, the coherent scattering (Rayleigh) and the incoherent scattering (Compton) attenuation coefficients, respectively.

$$
\mu_{\text {tot }}=\mu_{\mathrm{pe}}+\mu_{\mathrm{coh}}+\mu_{\text {incoh }}
$$

Note that there only are considered the interactions between radiation and matter that occur in the range of medical imaging energies.

\subsection{Radiographic acquisition}

Different tissues have different constituents and subsequently different physical properties. The variation of the attenuation coefficients between tissues is the basic principle of the radiology. The X-ray beam is attenuated of different forms by the different structures of the body. Consequently, the intensity of the resulting beam will be modelled according the thickness and attenuation coefficient of the crossed tissues. As a result, this resulting X-ray beam will interact, by an uneven form, with the materials of the film or of the detector. The grey levels of a radiogram are function of the number and the energy of the photons that arrive to the film or to the detector and, consequently, they are function of the attenuation caused by the tissues. 


\subsection{Hounsfield scale}

The Hounsfield scale is a range of grey levels, in which the grey value is associated to the attenuation experimented by the beam after crossing the tissues. It is useful for medical imaging because this scale distinguishes by the intensity of grey the different anatomical structures that have different attenuation coefficients [61].

The Hounsfield unit, $\mathrm{HU}$, is a dimensionless quantity and is defined by comparison with the attenuation coefficient of water. The $\mathrm{HU}$ is calculated as demonstrated in equation $3.6[61]$ :

$$
\mathrm{HU}=\frac{\mu_{\text {tissue }}-\mu_{\text {water }}}{\mu_{\text {water }}} \times 1000
$$

As reference, the values for air and water were defined as $-1000 \mathrm{HU}$ and $0 \mathrm{HU}$, respectively. On radiograms the pixels can be displayed on a range from -1024 to 3071 on the Hounsfield scale.

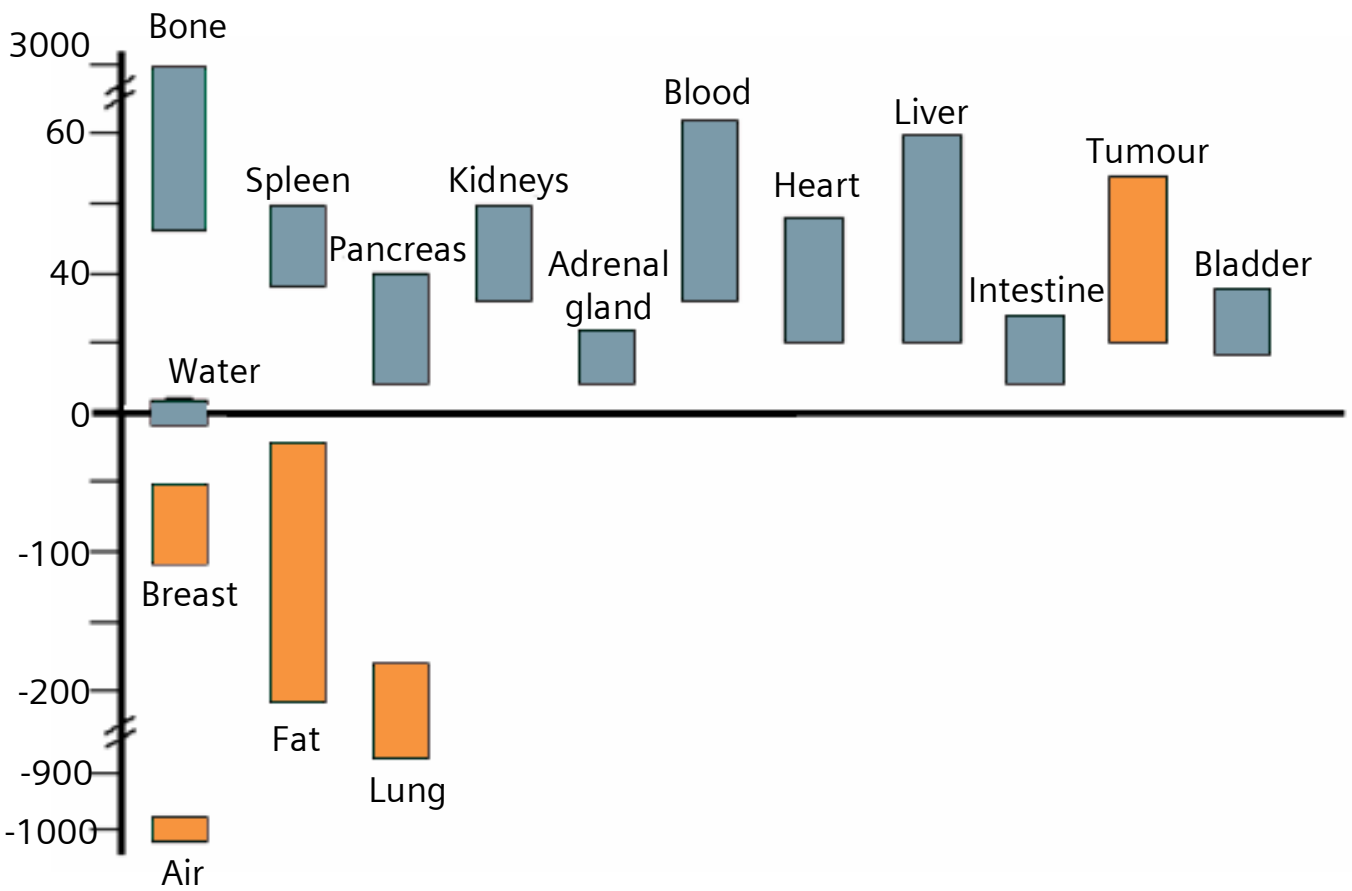

Figure 3.7 Hounsfield units range for different tissues. [62]

As the range is so large, the display window can be modified to set the grey scale to a small range of $\mathrm{HU}$ values that correspond to the tissue of interest. 


\subsection{Mammography}

The small differences between the attenuation coefficients of the breast tissues and the specific needs in the screening of breast diseases require the use of special equipments and techniques with low dose and high-quality images at low cost. The mammographic exam satisfies these requests.

The mammography is a radiographic exam, where a particular radiographic unit, the mammographic unit, is used to provide projections from a specific organ: the breast.

The X-ray beam generated penetrates the breast and a fraction of its photons interact with the air and with the tissues. The photons that were transmitted through the breast are recorded on a screen film or by a digital detector, in the case of conventional or digital mammography, respectively. Accordingly to the attenuation suffered during the trajectory between the source and the detector, an intensity image is created. The pixel intensity of the projection image is proportional to the energy and number of photons that reaches to the detector. The relation between the intensity of the photons that reach the detector and the attenuation coefficients is given by the Beer-Lambert law, as it was referenced.

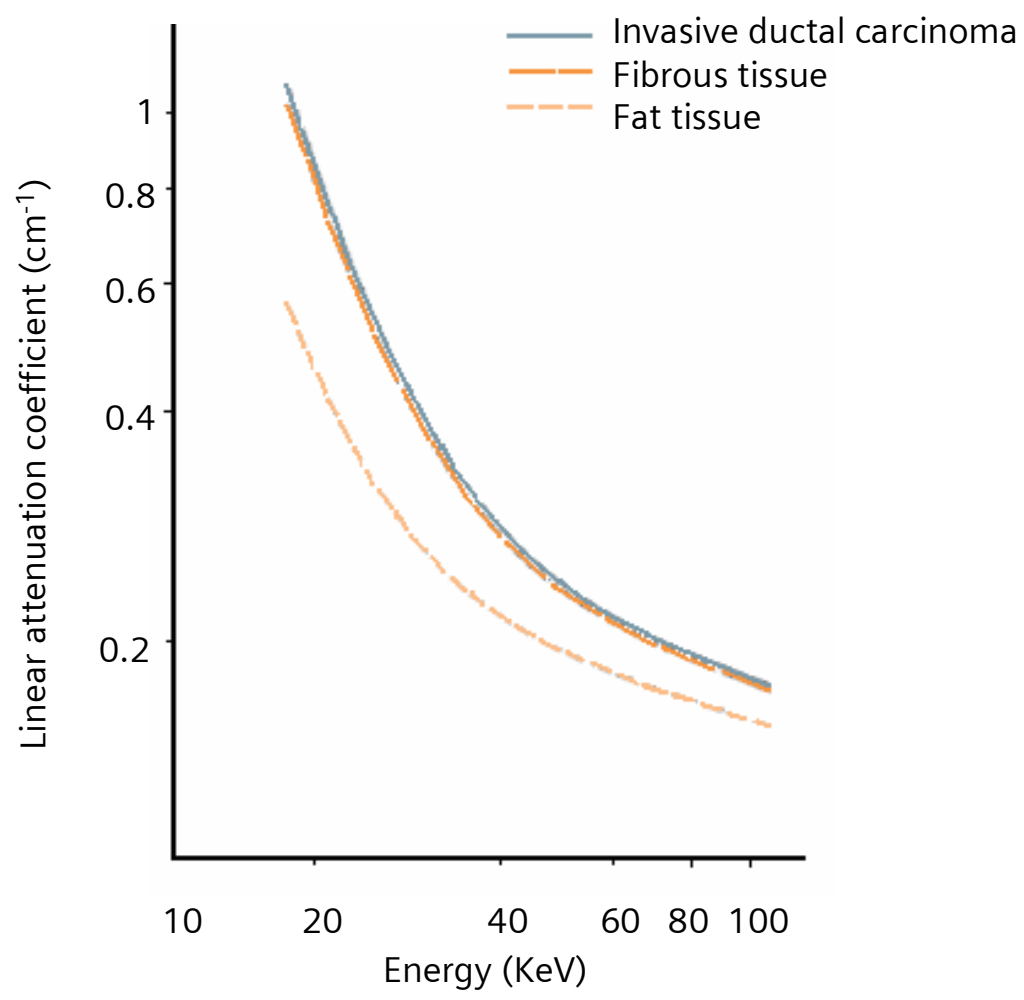

Figure 3.8 Mean values of the linear attenuation coefficient of invasive ductal carcinoma, fibrous and fat tissue. [63] 
The x-ray attenuation coefficients are very similar between tumour and fibroglandular tissues, limiting the distinction between them in the mammogram. As it can be seen in Figure 3.8, the x-ray attenuation coefficients of tumour, fibroglandular and fat tissue decrease with increasing energy, as their difference $[50,63]$.

Thereby, as the breast is mainly composed of fatty and fibrous tissues, which attenuate the photons less than the generality of the tissues, the range of the photons energies on mammography is low, around $20 \mathrm{KeV}$.

Figure 3.7 illustrates the principle of the mammographic acquisition, where the emitted X-ray beam crosses the breast and reaches the detector.

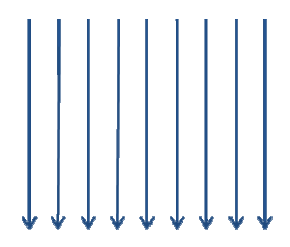

X-ray beam

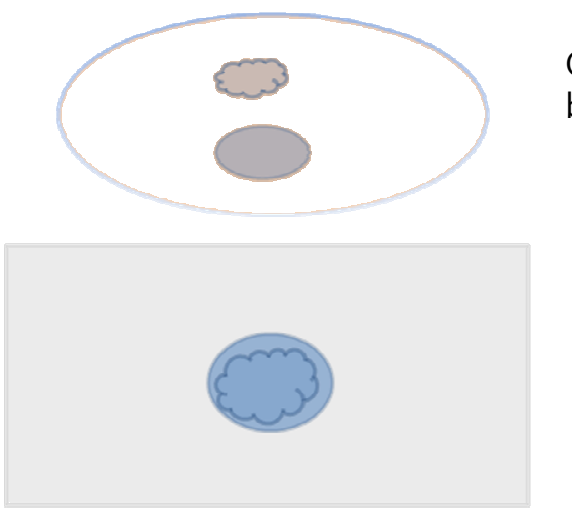

Objects on

breast

Projection

obtained on

mammography

Figure 3.8 Illustration of the obtaining of mammograms, evidencing the overlapping tissues.

The resulting image is just a projection of a volume. The superimposition of structures located in the same path of a group of photons may result in the concealment of these structures that are projected in the same area in the resulting image. This overlapping of structures brings uncertainty to the acquired mammograms.

Even so, mammography is the most used technique for screening and diagnostic of breast diseases, since it is an accurate technique that has a high specificity and sensitivity. These terms are defined below: 


$$
\begin{gathered}
\text { Sensitivity }=\frac{\text { patients with suspected breast cancer }}{\text { patients with histologically confirmed breast cancer }} \\
\text { Specificity }=\frac{\text { patients with suspected benign disease }}{\text { patients with histologically confirmed benign disease }} \\
\text { Positive predictive value }=\frac{\text { patients with histologically confirmed breast cancer }}{\text { patients with suspected breast cancer }} \\
\text { Accuracy }=\frac{\text { patients with true - positive and true - negative detected disease }}{\text { patients with histologically confirmed breast cancer }}
\end{gathered}
$$

In 2000, Poplack et al. presented a study, involving 53,803 women, with the aim of evaluate the mammography performance. The study revealed a sensitivity of $72.4 \%$ and $78.1 \%$, a high specificity of $97.3 \%$ and $89.3 \%$, and a positive predictive value of $10.6 \%$ and $17.1 \%$ (accordingly with the exam type: screening and diagnostic mammography, respectively)[15]. A study, done with 106,826 women, was completed to examine the screening mammography performance accordingly to the symptomatic status [18]. In order to compare mammography, ultrasonography and magnetic resonance, the sensitivity, specificity and positive predictive value were measured. $83.7 \%, 68.5 \%$ and $67.8 \%$ were the values of mammography, respectively [64]. More recently, Taplin et al. presented a study involving 237,669 women. In this case the sensitivity of the mammographic technique was $79.6 \%$, the specificity was $90.2 \%$ and the positive predictive value was $4.1 \%$ [16]. On a study about diagnostic mammography, $86.8 \%$, $98.6 \%$ and $68.8 \%$ were the values of sensitivity, specificity and positive predictive value, respectively [17]. The results were similar between these two studies, excluding the positive predictive value that had a wide range of values.

These results make the mammography the gold standard technique to the screening and diagnostic of breast cancer. Mammography is the lead technique for breast imaging, because of its precision. However, due the low positive predictive value, the recalls after the mammography are high and most often the lesion is benign.

Nevertheless, the capabilities of mammography are inevitable limited by the fact of 3D anatomical information being projected into a 2D image plan (Figure 3.7):

- Overlapping tissue that can mask tumours;

- Loss of specificity between cancerous, glandular and fibrous tissue;

- Loss of sensibility and specificity when the superimposed dense tissues look like a cancerous tissue. 


\subsection{Digital Breast Tomosynthesis}

As explained by values of specificity and sensibility, nowadays, the mammography is the gold standard to the screening and diagnosis of cancer and other diseases of breast. Though, it is often complemented with other techniques.

But even mammography has its limitations. The two-dimensional nature of mammography causes some problems, as the loss of specificity or the possibility to conceal cancer, for the reason that the signal detected by the receptor is dependent of total attenuation of all tissues above. Pathologies of interest can be hided by the clutter of signals from tissues above and below [46].

Besides the resulting problems of overlapping tissues, mammography is inefficient to young women, women with dense breasts or women with breast implants [65].

The Digital Breast Tomosynthesis is an innovative technique that inherits the best of mammography and can solve these problems. The system used acquire the images is similar to the mammographic unit (Figure 3.9). But the source moves around an arc to take the various projections.

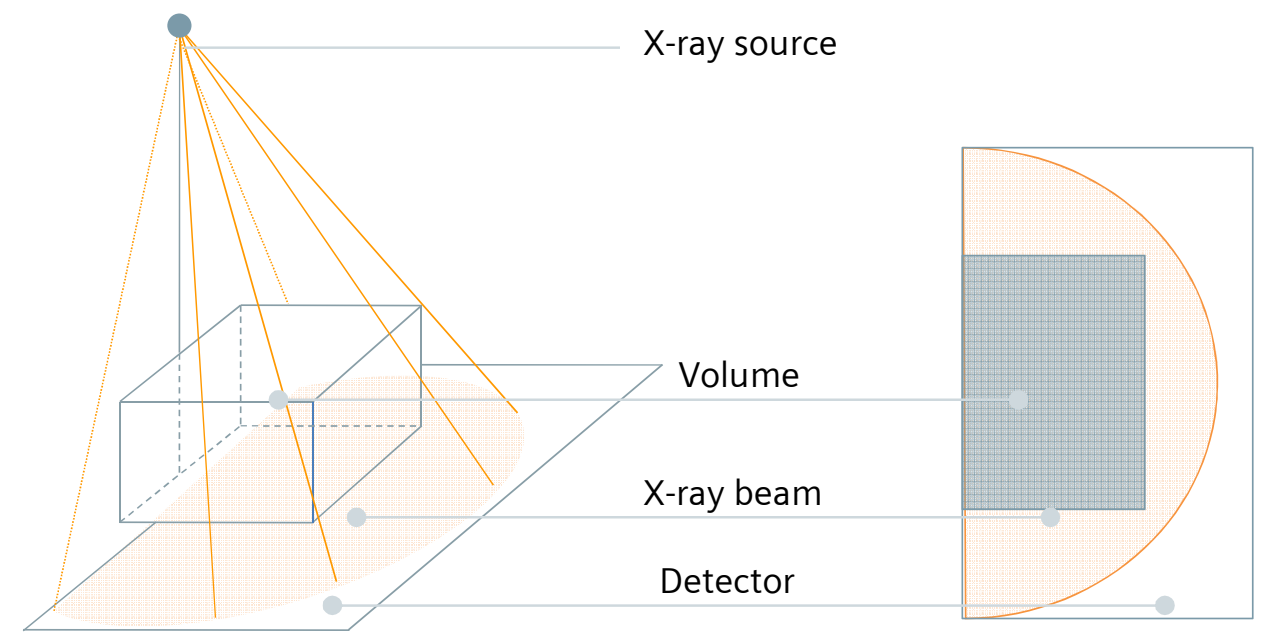

Figure 3.9 Diagram illustrating the tomosynthesis system, where the $x$-ray tube moves around an arc to take the projections.

The DBT is based in the principle of tomosynthesis. This is a technique used for decades, although never in the breast imaging, in which a few projections of an object are taken from different angles. Tomosynthesis differs from tomography by the limited number of projections taken and by the restricted angular range. 
In the DBT about eleven projections of the breast are obtained in an angular range of fifty degrees. The number of acquired images can be superior. However, the greater the number of projections, the higher the dose received by the organ. In order to guarantee a low dose received by the woman, only eleven images may be acquired, allowing a lower received dose in DBT than in mammography.

The individual images took separately can be computationally processed to form a series of high resolution slices, composing a 3D image. For this, specific algorithms for $3 \mathrm{D}$ reconstruction may be used and hence enable the examination slice to slice, seeing different plans without overlap. This technique will improve and make easier the screening and diagnosis. Figure 3.10 indicates how this technique will allow the distinction of different anatomic structures of the breast [66].
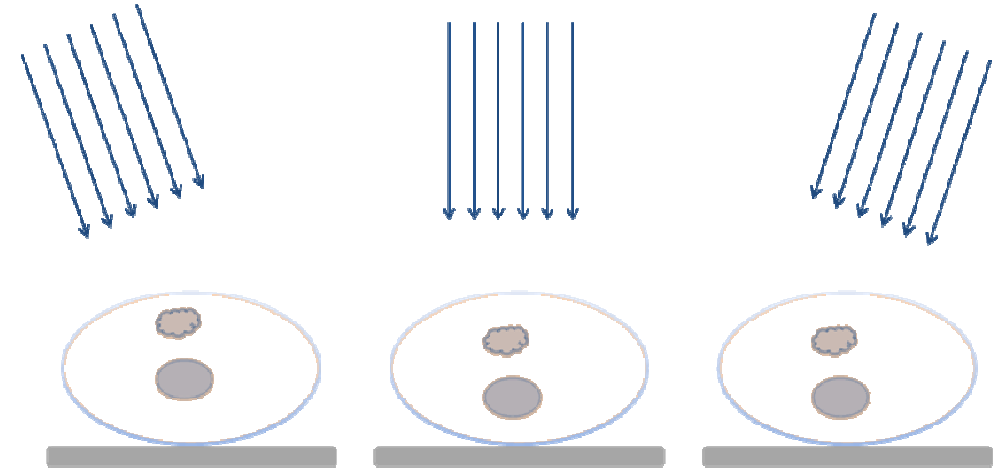

Objects on breast

Detector
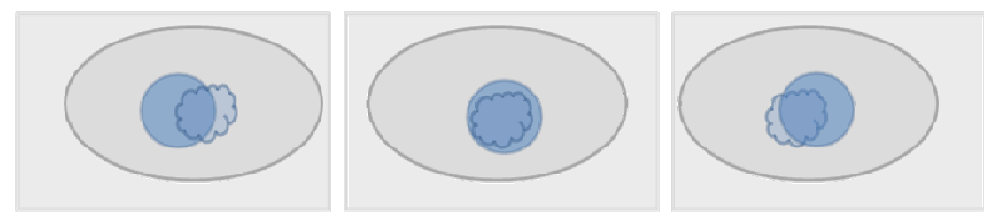

Projections on tomosynthesis

Figure 3.10 Schema of the image acquisition on different angles of the same object.

The reconstruction of the various projections will allow the distinction between different structures.

The various images acquired with this method are aligned and processed, allowing the visualization of a single slice without the interference of the tissues above and below of the same plan (Figure 3.11). A structure of interest can be evidenced only by selecting the plan in which it is located. The size, the shape, the margins and the location of a specific volume can be highlighted by this technique. 

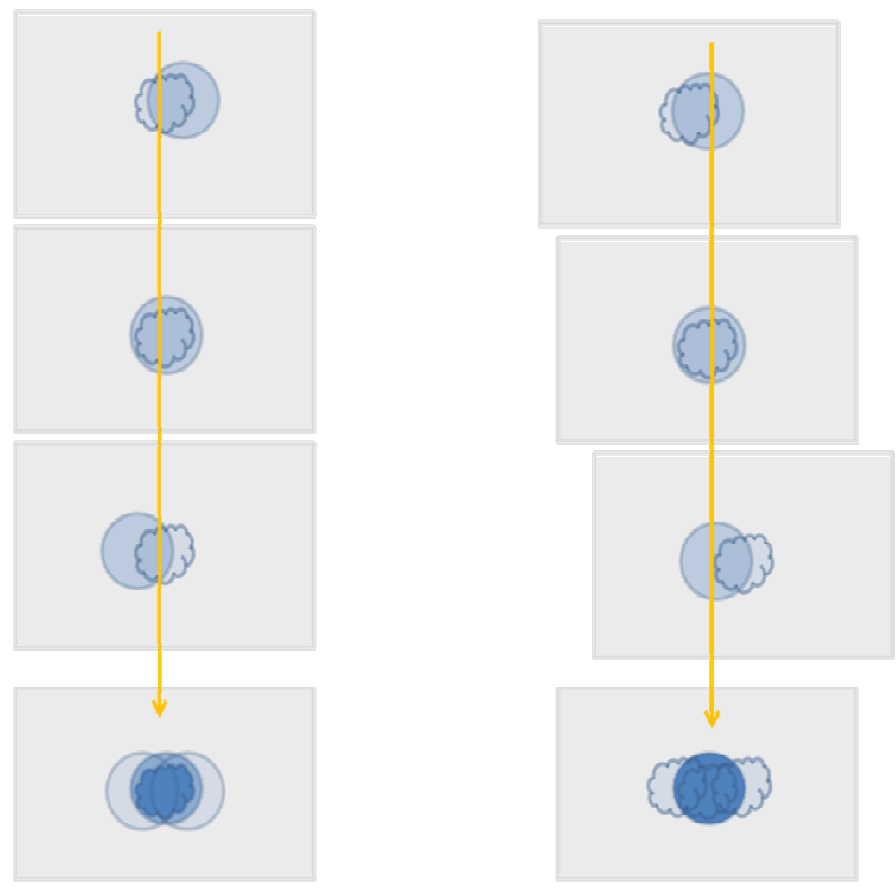

Figure 3.11 Image processing.

With these characteristics, tomosynthesis can bring many benefits to healthcare, as [46, 47, 67]:

- Allow the 3D visualization, solving the problems linked to the projection of overlapping tissues. The exam analysis will be done slice by slice, without the interference of the tissues above and below the analysed plan;

- Increase the specificity, allowing the distinction between malignant and benign tissue, since the morphology of a possible lesion is best perceived;

- Increase the sensitivity, allowing the analysis of the shape and size of the lesion from patients with suspected breast cancer;

- Analyze the lesion margin and get better knowledge of the extent of breast disease;

- Verify the correct localization of the lump, facilitating its removal in case of surgery;

- Reduce the number of biopsies performed, since it will increase the sensitivity and specificity, the certainty of the diagnosis will be greater;

- Decrease the compression of the breast, resulting on a greater comfort to the women. Only a slight pressure is needed to keep the breast in a static position and to prevent the blur associated to the motion of the patient; 
- Increase the contrast between tissues, facilitating the diagnostic and the distinction between structures;

- Improving the image of dense breasts, where the examination of mammograms is an arduous work. 



\section{Monte Carlo Simulations}

\subsection{Monte Carlo methods}

Monte Carlo simulation is a statistical method that uses repeated random (or pseudorandom) sampling to compute the behaviour of physic, mathematical or biological systems.

This numerical method is known since long, but only in last century it was recognized as a method suited for the most complex purposes when the application of deterministic algorithms is impossible. The technique owes its name "Monte Carlo" to a group of physicists during the Manhattan Project of World War II. The name is a reference to the Monte Carlo Casino, making an analogy between the randomness nature of the games of chance and the probabilistic nature of the problem related to the coefficient of diffusion of neutron to produce the neutron bomb.

This tool has a roll of areas of application where some processes have an associated uncertainty: physics, chemistry, biology and even sociology and economy [68]. 
One of these areas of application is medical physics in which Monte Carlo simulation is an important tool to assist the development and the improvement of medical imaging devices, to do dosimetric calculations, to assess image reconstruction algorithms and scatter correction techniques and to optimize scan protocols [69].

The Monte Carlo method does not solve an explicit equation. It only obtains an estimate of a concrete process by its simulation using the average behaviour, which is defined by the functions of distribution of probability of this process.

Monte Carlo simulations allow the estimation of physical parameters that are difficult to approximate on experimental measurements or analytical modelling. Some examples of application where Monte Carlo simulations are requested:

- Evaluation and optimization of design geometries and scanning parameters;

- Scatter characterization;

- Detector configuration and used materials;

- Absorbed dose calculations;

- Generation of data sets for testing reconstruction.

The Monte Carlo method can perform a good solution to model a radiographic acquisition, since the path of each individual photon is independent from every other particle and the distributions of probability that define the particle behaviour are well known. The production of photons, the interaction between them and the materials and theirs scatterings are simulated by sampling the functions of distribution of probability of each condition involving the use of random numbers [70].

Specifically on radiology and considering the stochastic nature of the process such as the interaction between the X-rays with tissues or the X-ray generation and detection, a tool as Monte Carlo methods is important create simulated mammograms to evaluate its reconstruction.

The definition of the geometries and dimensions of each element, the distance between them, the materials and the physical phenomena intervenient should occur as realistic as possible. Thus, the Monte Carlo simulation should occur on similar conditions to the real mammogram acquisition. The interactions between the radiation and the components of the system are calculated based on probabilities of occurrence [71]. 


\subsubsection{General-purpose source codes based on Monte Carlo methods}

There are various general-purpose source codes, based on Monte Carlo methods, to simulate the radiation transport through the matter, with application in medical and health physics [72].

Some of these platforms of simulation are bravely described below:

- Geant4 (for GEometry ANd Tracking) is a Monte Carlo code for simulating the transport of particles through the matter, in different contexts of physics: high energy physics, nuclear and accelerator physics, space and radiation, technology transfer and medical studies [73];

- The EGSnrc code (Electron Gamma Shower, National Research Council) was originated from EGS (Electron Gamma Shower) for high energy physics applications (the version of EGS create for SLAC - the Stan-ford Linear Accelerator Centre - is no longer developed). It is maintained by the Canadian National Research Council and Japan's High Energy Accelerator Research Organization [72];

- The MCNP code (Monte Carlo N-Particle) was developed by Los Alamos National Laboratory and is well-known by its applications on dosimetry [74];

- The FLUKA code (FLUktuierende KAskade), developed by INFN and CERN, has many applications in physics, including on Medical physics [75].;

- PHITS (Particle and Heavy Ion Transport Code System) is a transport code used on medical physics to simulate radiotherapy treatments [76].

\subsubsection{Geant4}

Developed by CERN on collaboration with world-wide scientists groups, Geant4 (for geometry and tracking) is a platform created to predict and describe the interactions between elementary particles and matter based on Monte Carlo calculations with a wide area of application in physics [73]. Geant4 software was developed on C++ programming language, using object oriented programming.

\subsubsection{GATE}

On the scope of this project, the GATE software is used to specifically simulate the acquisition of medical images, based on Geant4 $\mathrm{C}++$ libraries. 
GATE is widely used in nuclear medicine imaging research, since it was primordially developed for emission tomography. More recently, the last version already permits to simulate a CT scanner. This new and particular system is called CTscanner and is available on GATE Version 4.0.0.

On GATE the user interface is based on scripts and the actions can be performed entering commands in interactive mode or building up macro files containing the commands. Each command executes a specific function and thus they must be introduced in an orderly manner. The commands on the macro file define the different sections of a simulation: visualization; definitions of volumes; digitizer; physics; source and output.

A list of materials and their characteristics is defined on database available on GATE. The materials properties can be change, as well as new materials can be defined. These materials are used to define the different structures of the simulation.

CTscanner is a new system available on GATE used to perform the simulation of transmission tomography, specifically. Thus, it should be used to reproduce radiological exams, including the mammography.

\subsection{State-of-art of MC simulation in mammography}

Since CTscanner is a recent device, there are apparently no works performed on it. However, there are several experiments executed using Geant4 code. In the case of breast imaging, making a review of the literature, it is notorious that the Monte Carlo simulations are often used to analyse the influence of different materials in the exam quality.

Bonifácio et al. [77] used the Geant4 code to understand the behaviour of the diagnostic X-ray energy spectra, using different combinations targets, filters and tube voltages. A similar work has been completed by Ay et al. [78] but using MCNP4C code. Comparable studies were completed using ITS code [79] and MCNP4C code [80], also based on Monte Carlo methods.

Boone et al. [81] used MC simulations to analyse the theoretical performance of a fabricated anti-scatter grid for mammography. Mowlavi [82] used the MCNP code to calculate the X-ray spectra on mammography using different combinations of targets and filters. 
The MC methods are also often used to calculate the absorbed dose by the organs.

Delis et al. [83] developed a MC simulation program (DOSIS) oriented to mammography to perform dosimetric studies. The absorbed energy distribution was estimated under the influence of the tube voltage, the anode material, the filter material and the thickness. Later, the same group made another study about the influence of the tube voltage and filtration on the dose [84].

Dance et al. [85] simulated diverse mammographic exams, where different combinations of anodes and filters where placed. The aim was to establish the relation between the average absorbed dose and the image quality.

More recently, Sechopoulos et al. [86] used a program based on the Geant4 toolkit to calculate the dose in tomosynthesis of the breast, using different tungsten targets.

\subsection{Materials and Methods}

The elements which characterize the mammographic acquisition are introduced on GATE as realistic as possible. The main constituents to be described are the hardware of the mammographic unit and the phantom to represent the breast volume. The several aspects considered in their description are described on the following points.

\subsubsection{Mammographic unit}

The Siemens MAMMOMAT Inspiration is the digital mammography unit chosen to reproduce on the simulation platform (Figure 4.1). It was the chosen device because its features are similar to those required to perform a breast tomosynthesis exam. MAMMOMAT Inspiration:

- is a digital direct device;

- has a gantry that rotates up in a range from -25 to +25 degrees;

- has a X-ray tube that rotates up to +/- 180 degrees;

- is highly precise on the rotation control of the tube;

- enables fast and successive images acquisition by the detector.

The correspondence between technical specifications of MAMMOMAT Inspiration and the parameters introduced on GATE CTscanner can be seen in Table 4.1. 

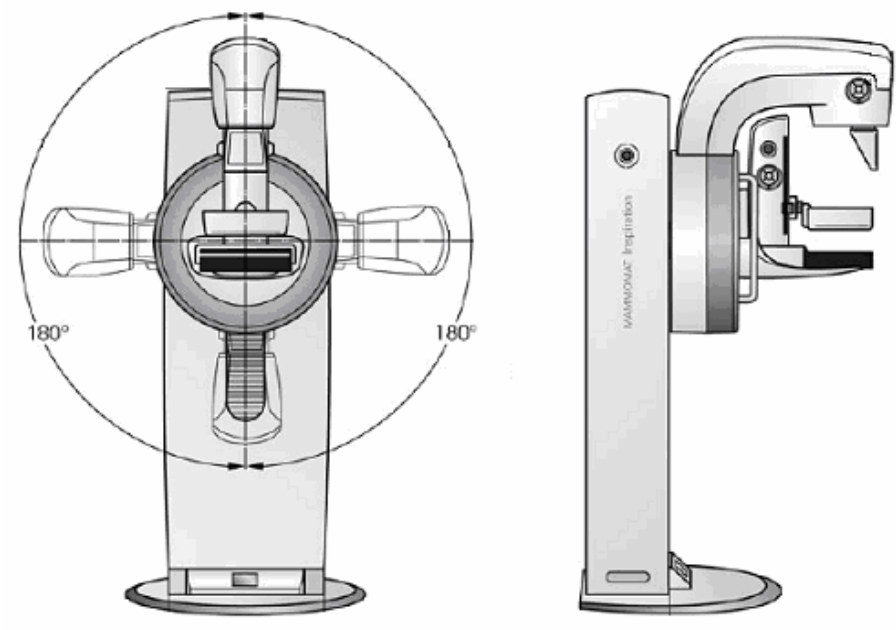

Figure 4.1 MAMMOMAT Inspiration design. [36]

Table 4.1 Correspondence between technical specifications of the mammography unit and the parameters introduced to perform the simulation.

\begin{tabular}{|c|c|c|}
\hline Component & Siemens MAMMOMAT Inspiration & GATE CTscanner \\
\hline Geometry & $\begin{array}{l}\text { Distance from X-ray tube to detector: } \\
65 \mathrm{~cm} \text { (extensible) }\end{array}$ & $\begin{array}{l}\text { Distance from source to } \\
\text { detector: } 67 \mathrm{~cm}\end{array}$ \\
\hline Detector & $\begin{array}{l}\text { Pixel size: } 0,085 \mathrm{~mm} \\
\text { Detector size: } 24 \times 30 \mathrm{~cm} \\
\text { Image matrix: } 2816 \times 3584\end{array}$ & $\begin{array}{l}\text { Pixel size: } 1 \mathrm{~mm} \\
\text { Detector size: } 24 \times 30 \mathrm{~cm} \\
\text { Image matrix: } 240 \times 300\end{array}$ \\
\hline $\begin{array}{l}\text { X-ray } \\
\text { generator }\end{array}$ & $\begin{array}{l}\text { Voltage: } 28 \mathrm{kV} \\
\text { Amperage: } 277 \mathrm{~mA}\end{array}$ & $\begin{array}{l}\text { Average photon energy: } 28 \mathrm{keV} \\
\text { Number photons per second: } 1.73 \times 10^{6}\end{array}$ \\
\hline Collimator & $\begin{array}{l}\text { Material: aluminum or lead } \\
\text { Window: } 30^{\circ} \\
\text { Dimensions: } 9.5 \mathrm{~cm}\end{array}$ & $\begin{array}{l}\text { Material: aluminum } \\
\text { Dimensions: } 9.5 \mathrm{~cm} \\
\text { Aperture: variable }\end{array}$ \\
\hline
\end{tabular}

Since on GATE is not possible to introduce the voltage and the amperage that give rise to the $\mathrm{x}$-ray beam, some auxiliary calculations are made to get the intensity of that beam and the average energy of the photons that compose it. The number of photons per second is only $1 \%$ of number of electrons per second present on the electronic flow. 
This number of electrons per second is directly linked to the amperage applied, as it can be seen in equation 4.1 .

$$
\text { number of electrons per second }=\frac{\mathrm{i}}{\mathrm{e}}
$$

Where $\mathrm{i}$ is the amperage and $\mathrm{e}$ is the electron charge and it is equal to $1.6 \times 10^{-19}$ coulombs. The average photon energy is dependent of the voltage applied, as it is demonstrated by equation 4.2 .

$$
\text { photon energy }=\mathrm{v} \times \mathrm{e}
$$

Where $v$ is the voltage applied. The values introduced on GATE are accordant with the usual values used on real acquisitions for breasts whose dimensions are similar to the phantoms employed.

\subsubsection{Geometry of beam}

On the Digital Breast Tomosynthesis device, the trajectories of the photons are not parallel between them, because the beam is composed by photons that are emitted along a polar angle from 0 to 20 degrees, performing an azimuthal angle of 180 degrees. These angles are displayed in Figure 4.2.
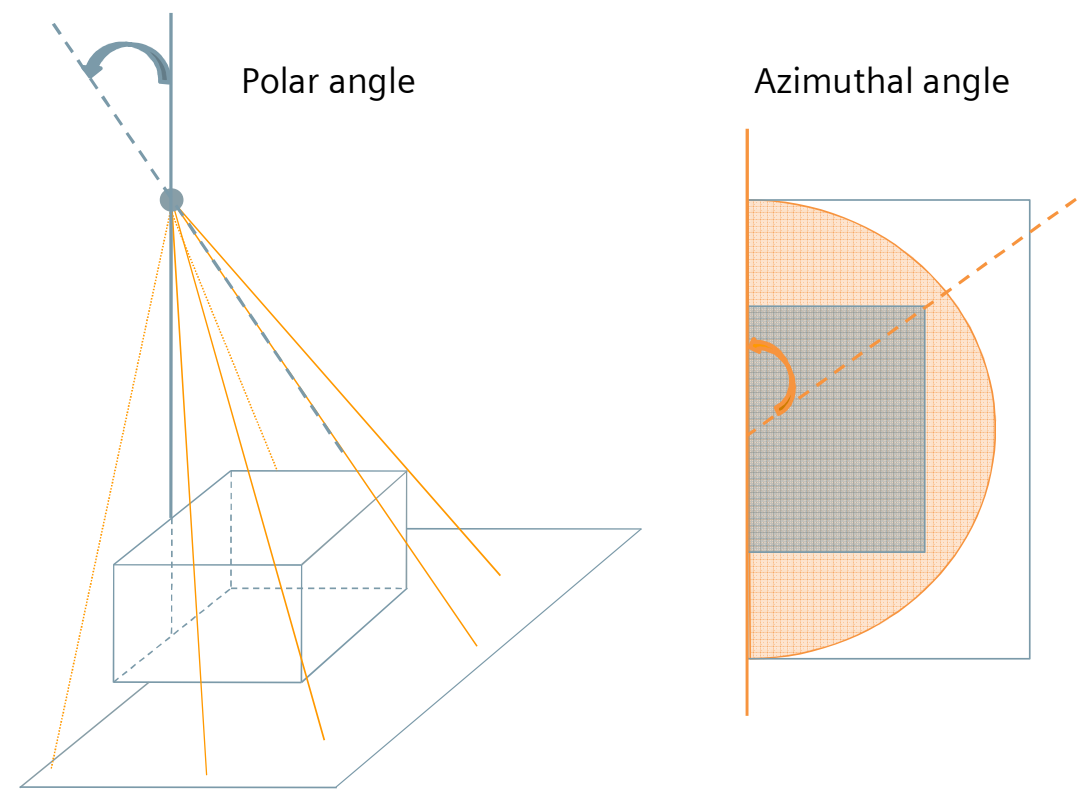

Figure 4.2 Diagram representing the geometry of the system, explaining the polar and the azimuthal angle. 


\subsubsection{Define a phantom on GATE}

The GATE platform allows the creation of volumes and their manipulation by the application of distinct commands. Inside the system, the volume can be defined and its attributes are then related, as its geometry, its placement, its motion, its material among others [69].

The volumes that represent the phantoms are defined by analytical functions making them important to the assessment of the image reconstruction, since theirs forms and appearance are well known. Moreover, the resulting images are more regular and stable, facilitating the evaluation of the image reconstruction in the earliest phases.

The mathematical phantoms are an important innovation in medical imaging. Real measurements can have, besides the algorithmic errors, other physical measurement errors. With an analytical phantom, the algorithmic errors can be isolated. Hence, these phantoms allow the creation of simulated and controlled data, the ideal data to access a reconstruction algorithm. These phantoms are created by analytical functions and each region is then assigned an attenuation coefficient [61].

The main point is that the reconstructed image can be compared with the exact model that is perfectly known from the beginning.

Besides the analytical phantoms, voxellized phantoms can also be defined on GATE. The voxellized phantom is a practical method to describe a volume which voxels vary in size and in material. This method allows the demarcation of different materials by the variation of the attributes of visualization, such as the colour of the material.

\subsubsection{Analytical phantom}

On the initial experiments completed on CTscanner, the components were defined on a simple manner, to shorten the simulation and to understand the mode of operation of the system. One of these experiments was performed defining the source, a collimator, the detector and a phantom. The characteristics of each element are described in Table 4.1. The phantom was a cylinder made of breast material, in which a smaller cylinder of silicon was introduced (Figure 4.3). 
Table 4.2 Characteristics of the compounds introduced in GATE to perform an acquisition.

\begin{tabular}{|c|c|c|}
\hline Compound & Compoun & \\
\hline Source & $3 \times 10^{4} \mathrm{ph}$ & \\
\hline Collimator & $\begin{array}{l}\text { Aluminum } \\
8 \times 8 \times 1 \mathrm{~cm} \\
5 \times 5 \times 1 \mathrm{~cm}\end{array}$ & sions) \\
\hline Detector & $\begin{array}{l}\text { Pixel size: } \\
\text { Detector s } \\
\text { Image ma }\end{array}$ & \\
\hline \multirow{3}{*}{ Phantom } & Cylinder & $\begin{array}{l}\text { Height: } 6 \mathrm{~cm} \\
\text { Radius: } 6 \mathrm{~cm} \\
\text { Material: Breast }\end{array}$ \\
\hline & \multicolumn{2}{|c|}{ Inside the major cylinder: } \\
\hline & 1 cylinder & $\begin{array}{l}\text { height of } 3 \mathrm{~cm} \\
\text { radius of } 1 \mathrm{~cm} \\
\text { Material: Silicon }\end{array}$ \\
\hline
\end{tabular}

The Breast and Silicon materials are part of the GATE material database. Once associated to a volume, GATE confers the existence of the material in its database and uses the material characteristics defined in there. The Breast and Silicon materials are already defined in the material list, where the material name has associated a density, the constituent elements and their individual abundances.

The phantom as it is described in Table 4.2 is shown in Figure 4.3. Only the outline of the major cylinder was drawn, to allow the visualization of the minor cylinder. 


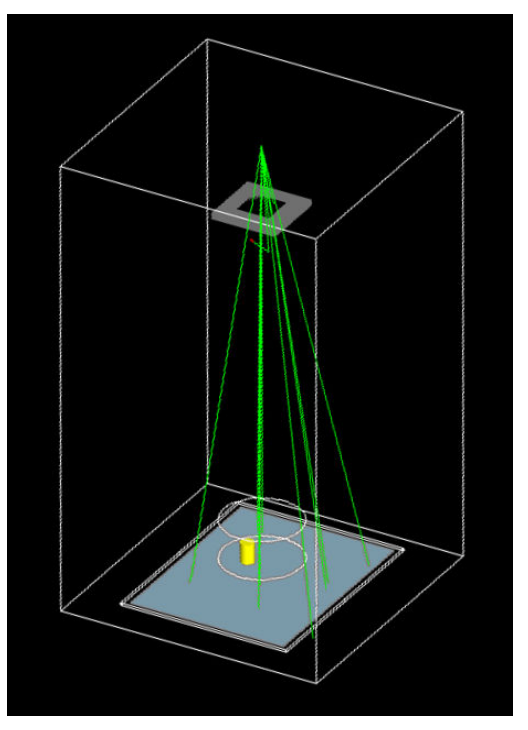

Figure 4.3 System completed in CTscanner with a cylindrical phantom made of breast material, in which a smaller cylinder made of silicon was introduced.

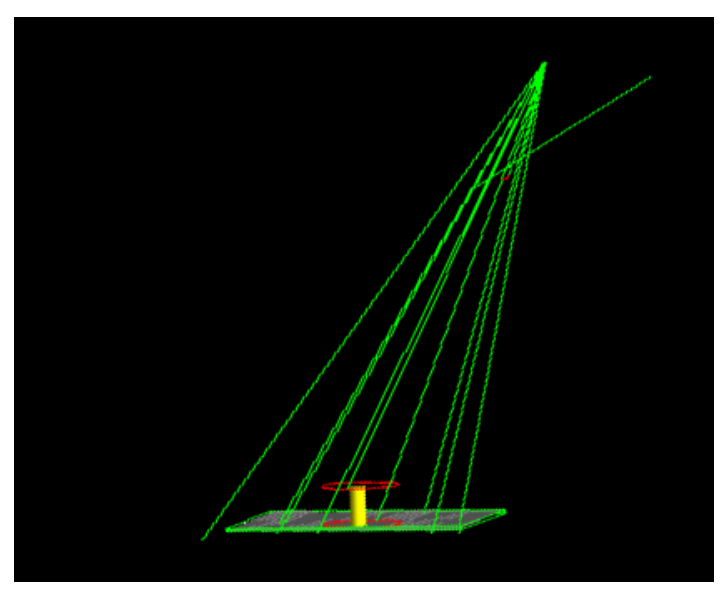

Figure 4.4 Picture of the GATE platform, showing the source dislocation.

Other parameters were defined to assemble the images for the reconstruction step. These images were also taken with analytical phantoms. The creation of eleven images of the same object from different angles composed by $240 \times 300$ pixels.

Table 4.3 presents the parameters that define the architecture of this simulation system. Various objects were introduced in the phantom to cause variations in the attenuation coefficients that allows a better assessment of the reconstruction methods performance. The architecture of this system allows the creation of eleven images of the same object from different angles, composed by $240 \times 300$ pixels. 
Table 4.3 Characteristics of the compounds introduced to perform the acquisition of images for reconstruction.

\begin{tabular}{|c|c|c|}
\hline Compound & Compound & racteristics \\
\hline Source & $\begin{array}{l}1731250 \mathrm{pr} \\
\text { Mono-eners } \\
\text { Polar angle: } \\
\text { Azimuthal a }\end{array}$ & $\begin{array}{l}\text { ns per second } \\
\text { photons of } 0.0279 \mathrm{MeV} \\
\text { degrees } \\
\text { e: } 180 \text { degrees }\end{array}$ \\
\hline Detector & $\begin{array}{l}\text { Pixel size: } 1 \\
\text { Detector siz } \\
\text { Image matr }\end{array}$ & $4 \times 30 \mathrm{~cm}$ \\
\hline \multirow{6}{*}{ Phantom } & Cylinder & $\begin{array}{l}\text { Height: } 50 \text { mm } \\
\text { Radius: } 50 \mathrm{~mm} \\
\text { Material: Breast }\end{array}$ \\
\hline & \multicolumn{2}{|c|}{ Inside the major cylinder: } \\
\hline & 3 cylinders & $\begin{array}{l}\text { Height: } 15 \mathrm{~mm} \\
\text { Radius: } 1 \mathrm{~mm} \\
\text { Material: Silicon }\end{array}$ \\
\hline & 1 ellipsoid & $\begin{array}{l}\text { Dimensions: } 10 \times 4 \times 4 \mathrm{~mm} \\
\text { Material: Weddellite }\end{array}$ \\
\hline & 2 spheres & $\begin{array}{l}\text { Radius: } 2 \mathrm{~mm} \\
\text { Material: Weddellite }\end{array}$ \\
\hline & 2 spheres & $\begin{array}{l}\text { Radius: } 1 \mathrm{~mm} \\
\text { Material: Weddellite }\end{array}$ \\
\hline Acquisition time & \multicolumn{2}{|c|}{3 seconds for each acquisition } \\
\hline
\end{tabular}

The dimensions that define the major cylinder approximate it to a small/normal breast. Inside of this major cylinder, other volumes were included to reproduce other different morphologies which can be encountered in breast. A mass was defined as an ellipsoid. 
The tumour masses have a very irregular shape, although the cysts have well-defined borders as an ellipsoid.

Four spheres were defined to imitate small calcifications and theirs dimensions were defined as a slightly larger than the pixels size [87].

The three smallest cylinders were defined to have a tubular shape as it happens with some calcifications [88].

The material that defines the major cylinder, the breast material, has a density of 1.020 $\mathrm{g} / \mathrm{cm}^{3}$. This value is defined by the program GATE and is a median density encountered in breast tissues. Although, this value can vary on a large range, from the fatty breast tissue to the fibrous breast tissue, it is a normal measure to use, and defines the homogeneous background of this phantom.

The material used to define the spheres was the Weddellite - $\mathrm{Ca}\left(\mathrm{C}_{2} \mathrm{O}_{4}\right) \cdot 2 \mathrm{H}_{2} \mathrm{O}$ which has a density of $1.94 \mathrm{~g} / \mathrm{cm}^{3}$. It has to be characterized in the GATE materials database, in order to be used to define some volumes. This material has calcium in its composition and it composes partly around $50 \%$ of the calcifications encountered $[87,89,90]$. This was the same material used to define the ellipsoid, since the calcium salts are also part of the malignant masses encountered in the breast tissue.

The silicon is a material which definition can also be encountered in the GATE materials database. Its density is $2.33 \mathrm{~g} / \mathrm{cm}^{3}$ and it was chosen to define the silicon wires, because it has higher density than the Weddellite, and can cause a higher contrast.

To acquire eleven projections of this phantom, the source moves around an arc of 50 degrees. Every 5 degrees an acquisition is performed to the same object, during 3 seconds. The phantom and the detector remain static along the simulation.

The phantom described in Table 4.3 is showed in Figure 4.5 as it is placed in the system to perform the simulation. 


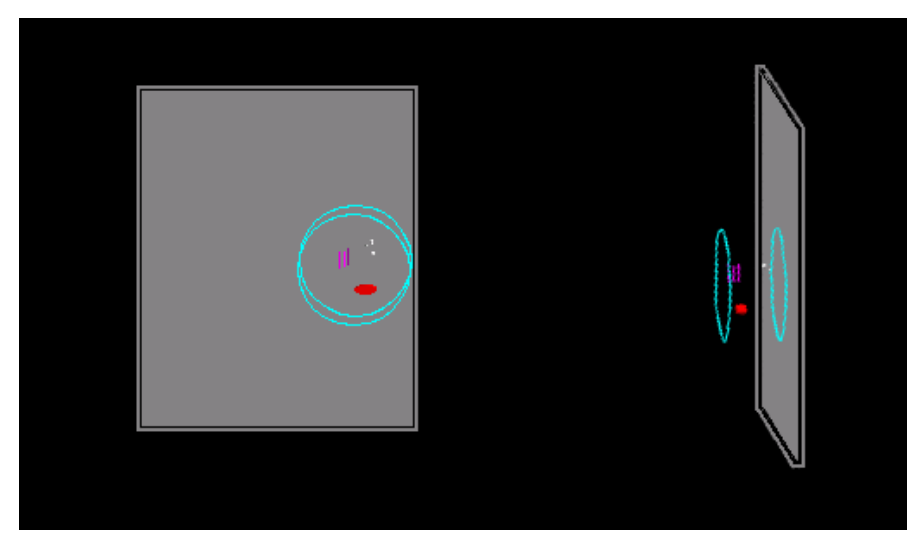

Figure 4.5 Cylindrical phantom in the simulation platform.

A schematic image of this phantom is illustrated in Figure 4.6 to give a better perception of the object localizations and to then compare with the reconstructed images.

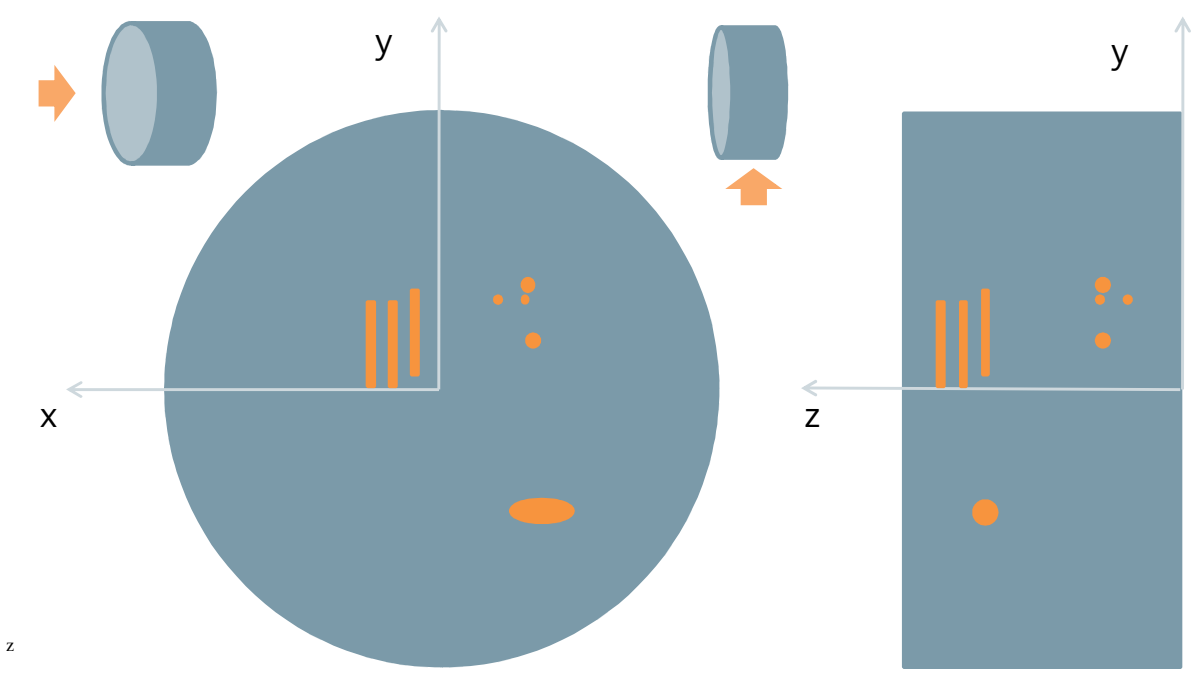

Figure 4.6 Diagram illustrating the position of the different objects inside the major cylindrical phantom.

The position of the different structures in the phantom is important to compare the reconstructed images with the original volume. Table 4.4 gives the coordinates of the elements, where the coordinate on $\mathrm{z}$ axis is special important to verify the artifact spread along the successive plans. 
Table 4.4 Coordinates of the different structures inside the major cylinder.

\begin{tabular}{|l|c|}
\hline Structure & Coordinates $(x, y, z)(\mathrm{cm})$ \\
\hline Ellipsoid & $(1.0,-2.0,3.5)$ \\
\hline Sphere 1 & $(1.5,2.0,1.5)$ \\
\hline Sphere 2 & $(1.0,1.7,1.7)$ \\
\hline Sphere 3 & $(1.5,1.7,1.0)$ \\
\hline Sphere 4 & $(1.6,1.0,2.0)$ \\
\hline Cylinder 1 & $(-0.5,1.0,3.5)$ \\
\hline Cylinder 2 & $(-0.9,0.8,3.9)$ \\
\hline Cylinder 3 & $(-1.3,0.8,4.3)$ \\
\hline
\end{tabular}

\subsubsection{NCAT phantom}

Developed by Paul Segars, Benjamin Tsui, E. Frey and E. Fishman [91], 4D NURBS-based Cardiac-Torso (NCAT) is a phantom that provides a realistic model of anatomy and physiology of human body. NCAT phantom is widely used on nuclear medicine imaging research, but it can also be applied on X-ray diagnostic techniques, since the phantom is used to define not only maps of biological activity distribution, but also maps of attenuation coefficient distribution.

In Figure 4.7, transversal slices of a female NCAT phantom are shown. These images are representations of the map of attenuation coefficient distribution obtained from NCAT program.

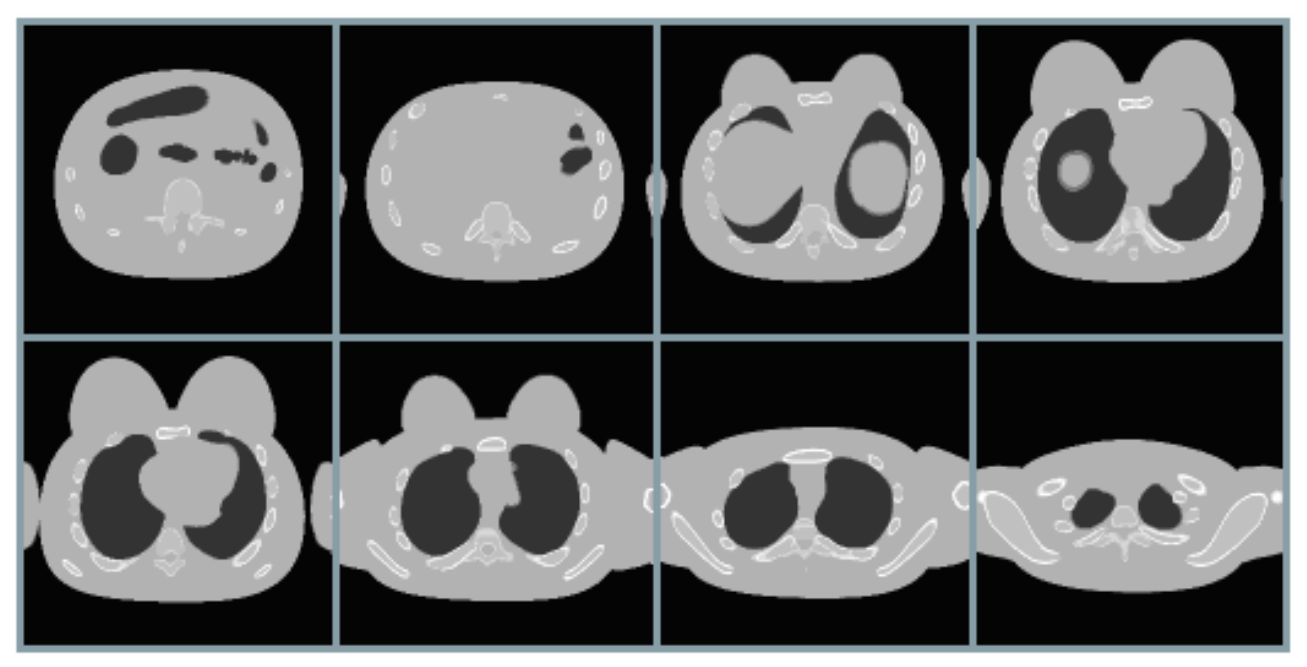

Figure 4.7 Transversal slices of a female NCAT phantom torso (from inferior to superior slices). 
In the aim of this work, to perform the simulation of mammograms, only the breast volume is needed. The NCAT phantom was utilized as a phantom of a normal woman, and then it was cropped and just the attenuation coefficient distribution map referent to the breast volume is used. Transversal, coronal and sagittal slices of this phantom breast are illustrated in Figure 4.8, Figure 4.9 and Figure 4.10, respectively.

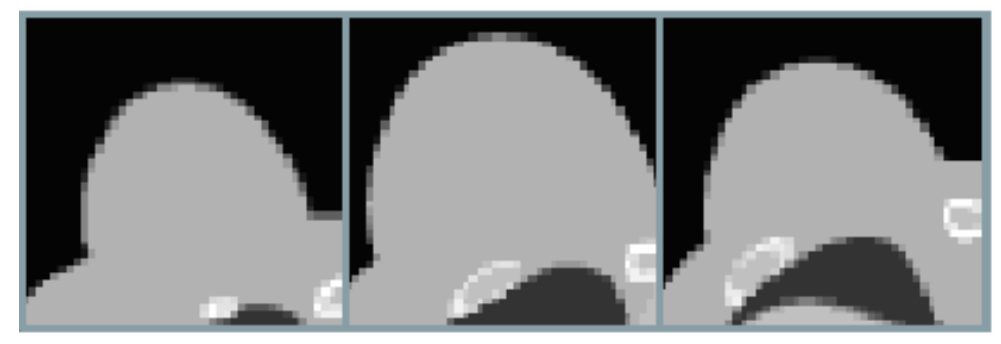

Figure 4.8 Transversal slices of the breast phantom (from superior to inferior).

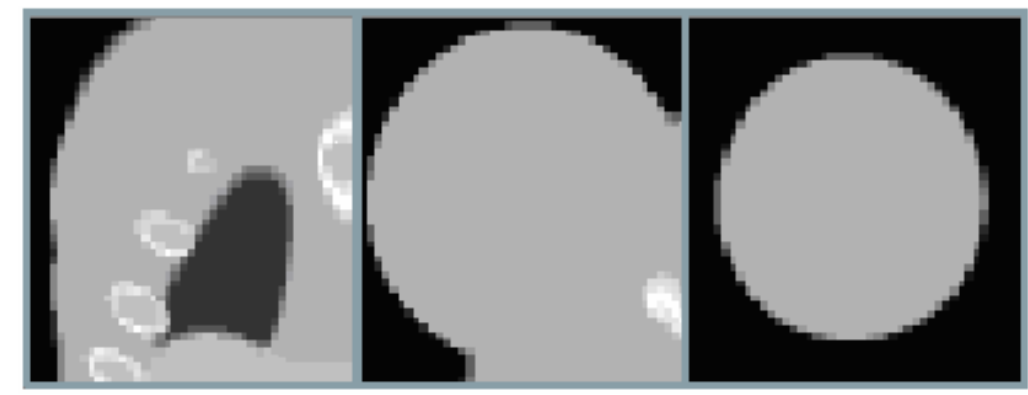

Figure 4.9 Coronal slices of the breast phantom (from anterior to posterior).

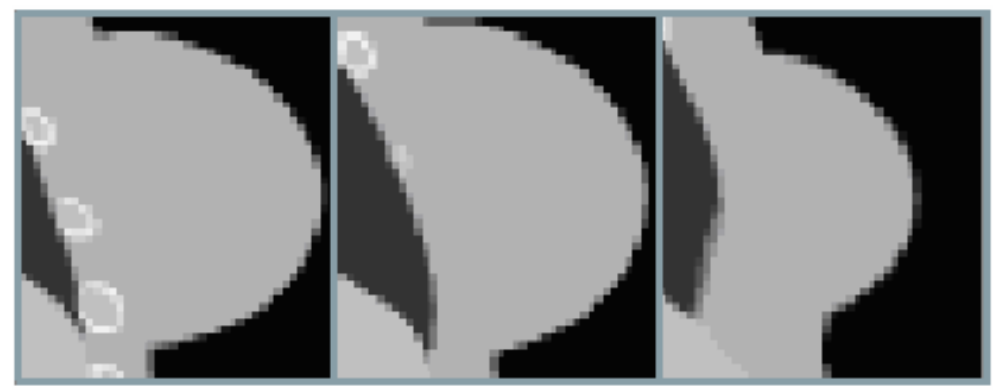

Figure 4.10 Sagittal slices of the breast phantom (from right to left).

On the GATE platform, the NCAT phantom is inserted as a voxellized phantom (Figure 4.11), being defined voxel by voxel and considering the material of each voxel: breast, bone, lung or air. Each material can be linked to a different colour to allow distinguish of the material in the GATE platform, as it is shown in Figure 4.11. 


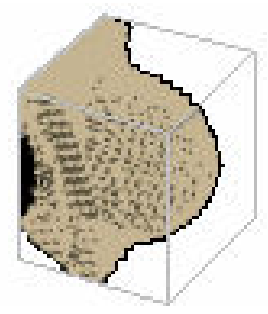

Figure 4.11 The breast volume of NCAT phantom as it is presented in GATE.

Table 4.5 present the various compounds defined in the system and theirs characteristics. The NCAT phantom is used to acquire more realistic simulated mammograms.

Table 4.5 Characteristics of the compounds introduced in GATE to perform an acquisition with the used of the voxellized phantom, the NCAT phantom.

\begin{tabular}{|c|c|}
\hline Compound & Compound characteristics \\
\hline Source & $\begin{array}{l}1731250 \text { photons per second } \\
\text { Mono-energetic photons of } 0.0279 \mathrm{MeV} \\
\text { Polar angle: } 13 \text { degrees } \\
\text { Azimuthal angle: } 180 \text { degrees }\end{array}$ \\
\hline Detector & $\begin{array}{l}\text { Pixel size: } 1 \mathrm{~mm} \\
\text { Detector size: } 24 \times 30 \mathrm{~cm} \\
\text { Image matrix: } 240 \times 300 \text { pixels }\end{array}$ \\
\hline Phantom & Breast from the NCAT phantom \\
\hline Acquisition time & 3 seconds for each acquisition \\
\hline
\end{tabular}

This phantom placed in the GATE system, during an acquisition from the 0 angle, can be visualized of Figure 4.12. 


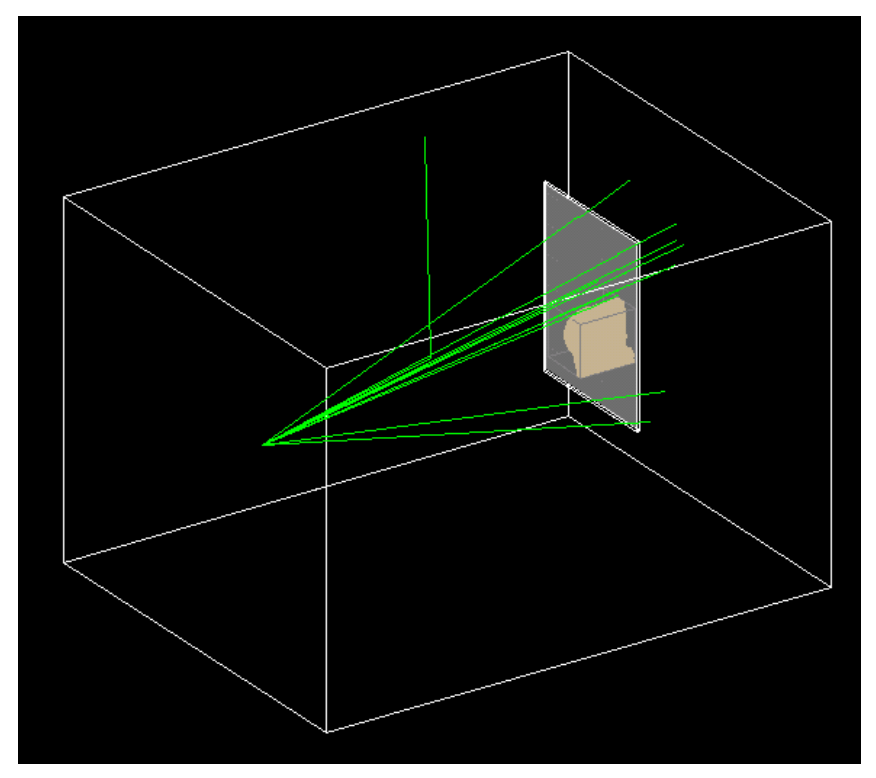

Figure 4.12 Picture showing a mammographic acquisition in GATE. 



\section{Image reconstruction}

\subsection{The problem of image reconstruction}

The problem of image reconstruction is to reconstruct the region of interest, i.e. to know the function $x$, from its projections taken along the different directions. This is considered an inverse problem since the information of interest, in this case the distribution of the attenuation coefficient, is not directly available: we want to know the object of interest, knowing the projection given by the device.

\subsubsection{Radon transform}

The Radon transform has many applications besides its application in medical imaging, as in radar imaging or geophysical imaging.

In medical imaging, the Radon transform is a function which computes several projections (line integrals) of an image matrix along parallels or beams paths in specified directions. To represent the image, the Radon transform takes multiple 
projections from different angles around the image, where function $\mathrm{x}$ is an unknown density, Figure 5.1.

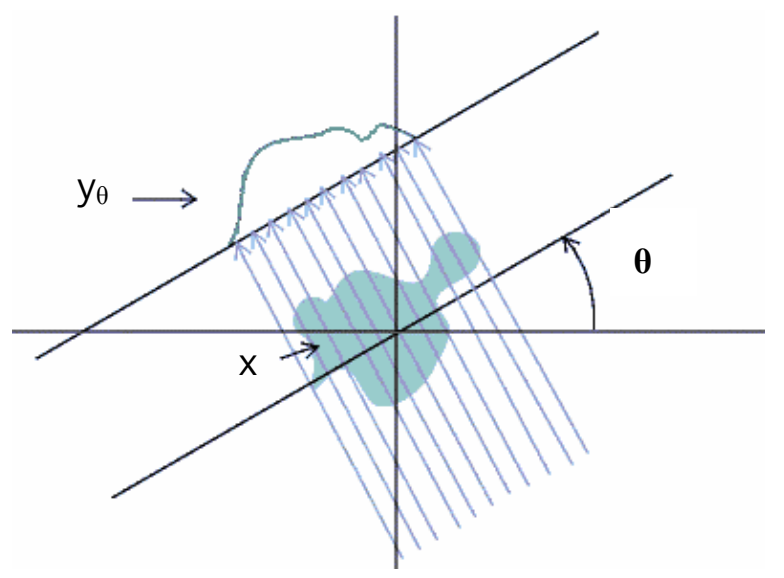

Figure 5.1 The 1D projection at angle $\theta, y_{\theta}$ is the integral of the object distribution $x$.

The distribution $f$ is the input that we want to estimate.

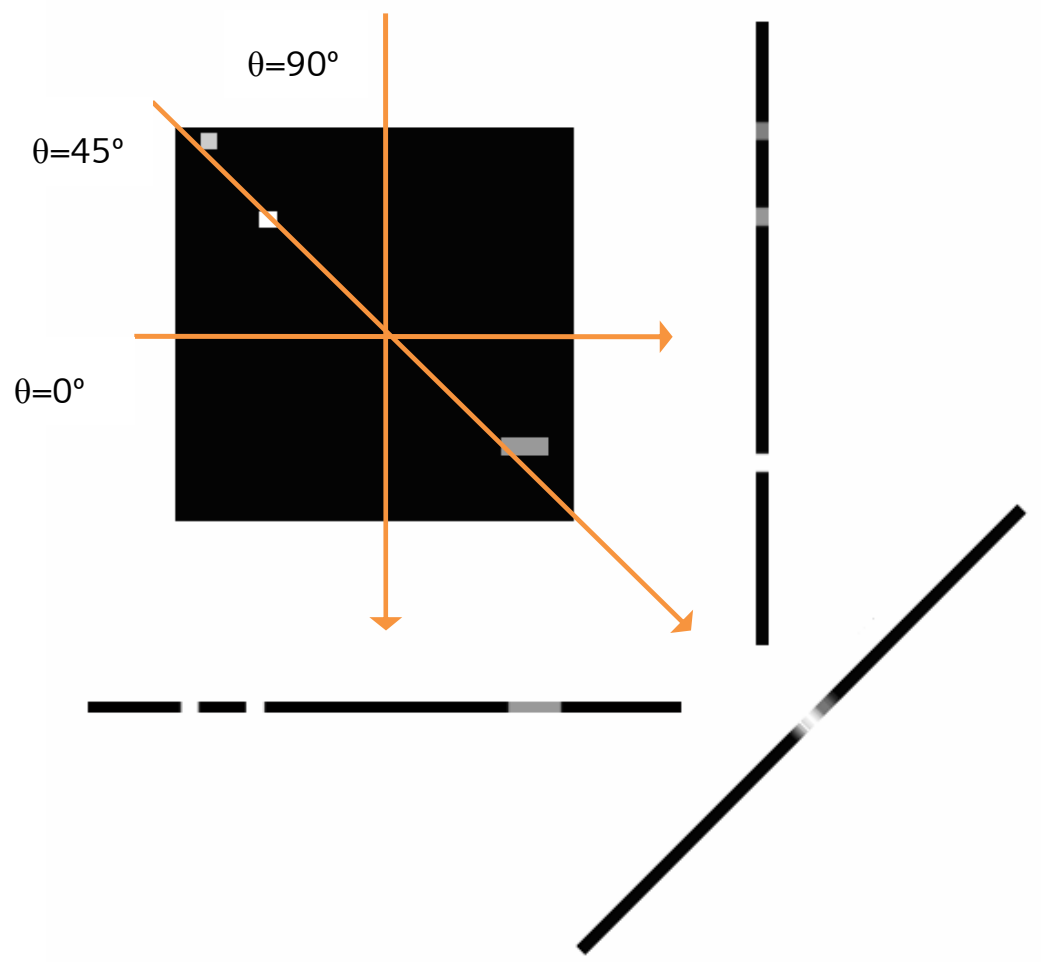

Figure 5.2 Different projections of the original object.

This problem can be solved using the inverse Radon transform that estimates the original image from its projections taken along different directions (Figure 5.2).

One of the limitations in the tomosynthesis reconstruction is the restricted range of angles used to acquire the projections. In DBT, the angle range is from -25 to 25 
degrees. The effects of this limitation in the resulting image are demonstrated in Figure 5.3, where the inverse Radon transform was used to restore the original image presented in Figure 5.2 using angle ranges of 50, 90, 180 and 360 degrees.
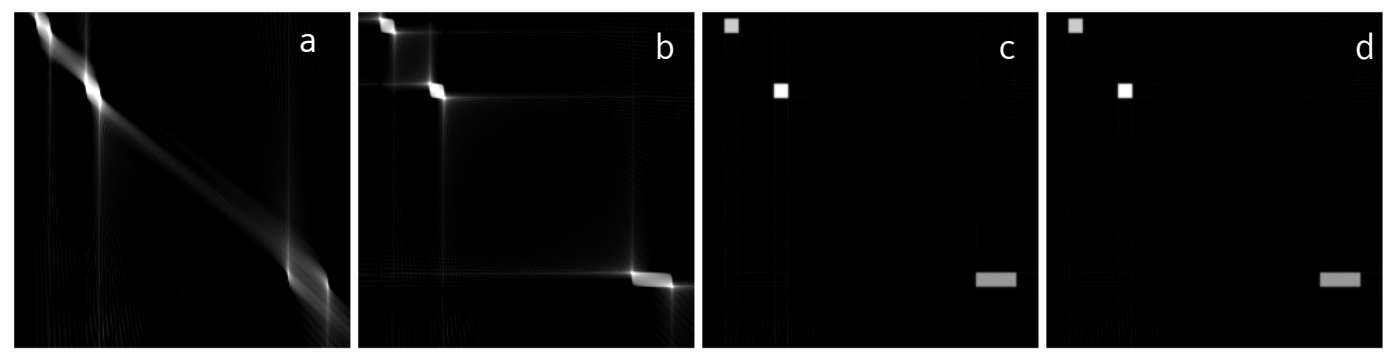

Figure 5.3 Image reconstruction using the inverse Radon transform taking an angle range of 50,90, 180, 360 degrees, a, b, c and d, respectively.

\subsubsection{System Matrix}

Another way to estimate the attenuation coefficient distribution along the body, is constructing a system matrix. This matrix is an estimative to the form how the voxel contributes for the attenuation of the beam. It is the model of the photon transmission process.

The process of image reconstruction must be accordant to the particular geometry of the system of the Digital Breast Tomosynthesis. The plan detector and the geometry of the beam must to be considered during the image reconstruction. The system matrix was constructed accordantly with this specific geometry.

Given this geometry, the implementation of a system matrix was an advantage instead the construction of sinogram and the subsequent use in the STIR program, which was the initial option for this project. The sinogram structure is generally implemented for cylindrical geometries and, in this case, it was difficult to construct the sinogram.

The system matrix calculation must to be done for each angle of x-ray tube rotation. However each one of these matrix can be saved just once and then used several times for the interactive reconstruction methods with the same geometry.

In a general way, the complexity of the image reconstruction can be traduced on a difficulty of determine the distribution function, $x$, from equation 5.1. Here $y_{n}$ are the detector pixels values taken in the corresponding projection data $n, x$ is the real linear attenuation coefficient distribution in the original object and $A_{n}$ is the system matrix for the slice image of interest $n$. 


$$
y_{n}=A_{n} x
$$

$A$, the system matrix, is a $2 \mathrm{D}$ matrix composed by non-negative elements that describe the probability of a photon that encounters a voxels be absorbed. For each projection data, a system matrix is built (equation 5.2).

$$
\left[\begin{array}{c}
\mathrm{y}_{1} \\
\vdots \\
\mathrm{y}_{\mathrm{N}}
\end{array}\right]=\left[\begin{array}{c}
\mathrm{A}_{1} \\
\vdots \\
\mathrm{A}_{\mathrm{N}}
\end{array}\right] \cdot \mathrm{x}
$$

$\mathrm{N}$ is the total number of projections and $1 \leq \mathrm{n} \leq \mathrm{N}$.

\subsection{State-of-art of 3D image reconstruction in tomosynthesis}

The earliest algorithms applied to perform 3D reconstruction in tomosynthesis were derived from the back projection (BP) algorithm. The results suffered from blur from overlying objects in the plan of interest and limited the contrast enhancement of the slices. Today, there are more algorithms developed and improved methods to perform tomosynthesis reconstruction [92]. However, since the tomosynthesis for breast imaging is a recent device, the methods for 3D reconstruction are not optimized yet. In these last years, several groups of scientists tried to implement and assess the tridimensional image reconstruction in tomosynthesis.

Suryanarayanan et al. [93] compared four algorithms with digital planar mammography. Anyone of the four reconstructions algorithms: tuned aperture computed tomography (TACT)-BP, TACT-iterative restoration, expectation-maximization (EM) and Bayesian smoothing, showed a better contrast detail than digital mammography. A similar study was then performed by Suryanarayanan et al. [94] with the purpose of compare digital mammography with both linear and nonlinear tridimensional reconstruction methods: TACT-BP, TACT-maximization and TACTminimization. Beyond the conclusion already drawn (tomosynthesis methods outperformed mammographic methods), some differences were detected between the tomosynthesis methods. TACT-BP registered some blur, but it evidenced more details than the other algorithms. TACT-maximization reduced the blur, but suppressed fiducial markers. TACT-minimization reduced the blur as well as the signal-to-noise ratio. The 
authors suggested that a combination of linear and nonlinear reconstruction schemes may improve the image visualization.

Wu et al. [95] gives a thorough evaluation of tomosynthesis using a maximumlikelihood expectation-maximization (ML-EM) iterative reconstruction algorithm. The visibility of structures is improved on tomosynthesis comparing with mammography, but these results required 2 hours of computation time. Later, Wu et al. [96] used a parallel reconstruction method, based on maximum-likelihood (ML) algorithm. The serial and the parallel reconstruction were compared, decreasing the computer time from approximately 2 hours to few minutes (3.5 to 6.5 minutes). According to the authors, no significant differences were found between the two reconstruction methods.

Sompel et al. [97] used ML reconstruction algorithm simultaneously with a segmentation method. The resulting algorithm appears promising considering the edge preservation and the noise suppression, but it fails the reconstruction of small structures of the phantom.

Bleuet et al. [98] used Algebraic Reconstruction Technique (ART) and Half Quadratic Regularization (HQR) methods to improve the vertical resolution of shift and add (SAA) algorithm. In accordance with the author, ART improved the vertical resolution and HQR reduced the truncation artefacts.

Chen et al. [99] compared the performance of four techniques on digital breast tomosynthesis. SAA, Niklason algorithm, filtered back projection (FBP) and matrix inversion tomosynthesis (MITS) algorithms were compared analyzing the sharpness of the reconstructed structures and the effectiveness at removing the blur. MITS and FBP were superior to SAA and Niklason algorithms for removal of out-of-plan blur. Moreover, MITS was better than FBP, but they were similar for removal of blur from more distant plans. Afterward, Chen et al. [100] studied four algorithms: 1) MITS blended with SAA; 2) FBP; 3) FBP with applied Hamming and Gaussian filters, and 4) Gaussian Frequency Blending (GFB) of MITS and FBP. Results showed that MITS was better for high frequency content and FBP was better for low frequency content. FBP with Hamming and Gaussian filter reduced the high frequency noise, but introduced blur to the margins of micro-calcifications. GFB kept the good performance for low frequency content from FBP and the good performance for high frequency content from MITS. 
Zhou et al. [101] investigated the EM, simultaneous algebraic reconstruction technique (SART) and BP. The methods were chosen because they are an example of a statistical reconstruction technique, a deterministic linear inversion technique, and a simple and fast algorithm to implement, respectively. The results showed that EM was superior to SART, and both had a significantly higher performance than BP method.

Zhang et al. [102] compared three representative reconstruction methods for breast tomosynthesis using several phantoms: the BP method, the SART technique and the ML with the convex algorithm (ML-convex). For phantoms with homogeneous background, the BP presented smooth images with low background noise, while SART and MLconvex amplified the noise, but enhanced the contrast and edges of the features. These iterative methods can reduce the inter-plan blurring and artifacts. For a contrastdetail phantom, the BP method evidenced blurring artifacts, while the SART and the MLconvex can improve the object distinction by removing the overlapping structures. Zhang et al. concluded that all methods can reconstruct the breast structures in their correct plans and remove the overlapping tissues.

Sidky et al. [103] developed an iterative algorithm based on image total variation (TV) minimization, The new algorithm, the total p-norm variation (TpV) algorithm, was compared with the previous TV algorithm and with ML-EM. According to the authors the TpV improves substantially the efficiency when compared with the TV method. When TpV is compared with the ML-EM, it does not present improvements on the image quality, in particular for masses. But it may have advantages when imaging microcalcifications.

Summarizing, the reconstruction methods already tested for Digital Breast Tomosynthesis are:

- ART;

- BP;

- EM-ML;

- FBP;

- MITS;

- SAA;

- TACT;

- SART;

- TVP. 


\subsection{Materials and Methods}

\subsubsection{System Matrix calculation}

The whole volume to be reconstructed can be divided into $V$ connecting cubic voxels. Each voxel has an attenuation coefficient to be estimated, $\mathrm{xv}(\mathrm{v}=1,2, . ., \mathrm{V}$; where $\mathrm{V}$ is the total number of voxels). Each pixel from the projection data represent a value that is the line integral along a ray path, $y_{p}(p=1,2, \ldots, P$; where $P$ is the total number of pixels).

To each detector pixel, $\mathrm{p}(1 \leq \mathrm{p} \leq \mathrm{P})$, corresponds a straight segment that distances from this pixel to the $x$-ray source. Every voxel that is crossed by the straight segment contributes to the attenuation of the photons that reach the detector pixel, as it is illustrated in Figure 5.4 .

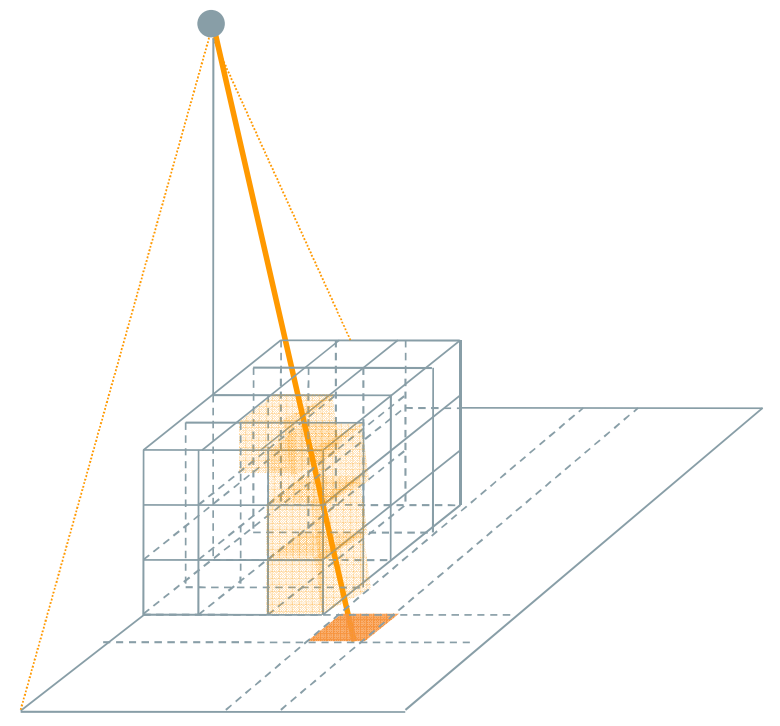

Figure 5.4 Diagram illustrating that the ray is attenuated only by the voxels that are crossed by it.

The whole volume is subdivided into $V$ voxels, $(1 \leq v \leq V)$, whose linear attenuation coefficient, $\mathrm{xv}$, will be estimated. The probability of a voxel to attenuate the photons is estimated accordantly to the length of the straight segment within the voxel. This is only one of the possible approaches, allowing pondering the contribution of each voxel to the total attenuation of a beam. Other approaches can estimate the attenuation by a voxel, for example considering the rays that reaches the same detector pixel as a cone, and using the whole volume of it inside the voxel. 
The method used is illustrated in Figure 5.5, where the intersection between a thin beam and a voxel is seen on a $2 \mathrm{D}$ projection.

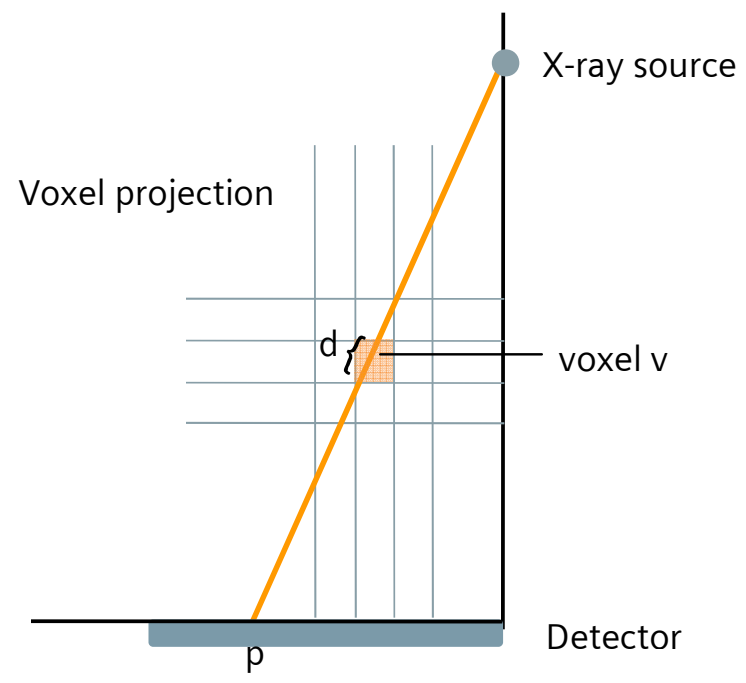

Figure 5.5 Diagram illustrating the intersection between a thin beam and a voxel on

2D.

If $\mathrm{x}_{1}, \mathrm{y}_{1}$ and $\mathrm{z}_{1}$ were the coordinates of the point where the thin beam intersects the frontiers of the voxel $v$, and $x_{2}, y_{2}$ and $z_{2}$ were the coordinates of the point where the thin beam gets out of the voxel $v$ (see Figure 5.6); then $d_{v p}$, the length of the segment that reaches $p$ within that voxel $v$, is given by equation 5.3 .

$$
\mathrm{d}_{\mathrm{vp}}=\sqrt{\left(\mathrm{x}_{1}-\mathrm{x}_{2}\right)^{2}+\left(\mathrm{y}_{1}-\mathrm{y}_{2}\right)^{2}+\left(\mathrm{z}_{1}-\mathrm{z}_{2}\right)^{2}}
$$

This distance is calculated for each combination of pixel and voxel, this is, for each pixel one straight segment is considered, that distances from the source $\left(F_{x}, F_{y}, F_{z}\right)$ to the pixel $\left(P_{x}, P_{y}, P_{z}\right)$, as it can be seen of Figure 5.6. The attenuation of this thin beam is made by every voxels that are crossed by it. Hence, to each voxel is given a value accordantly with dvp.

This calculation is made for each position of the source; this is, for each projection. Hence, there are eleven system matrices (as there are eleven projections of the same object), whose dimensions are $V \times P$, the total number of voxels by the total number of pixels. 


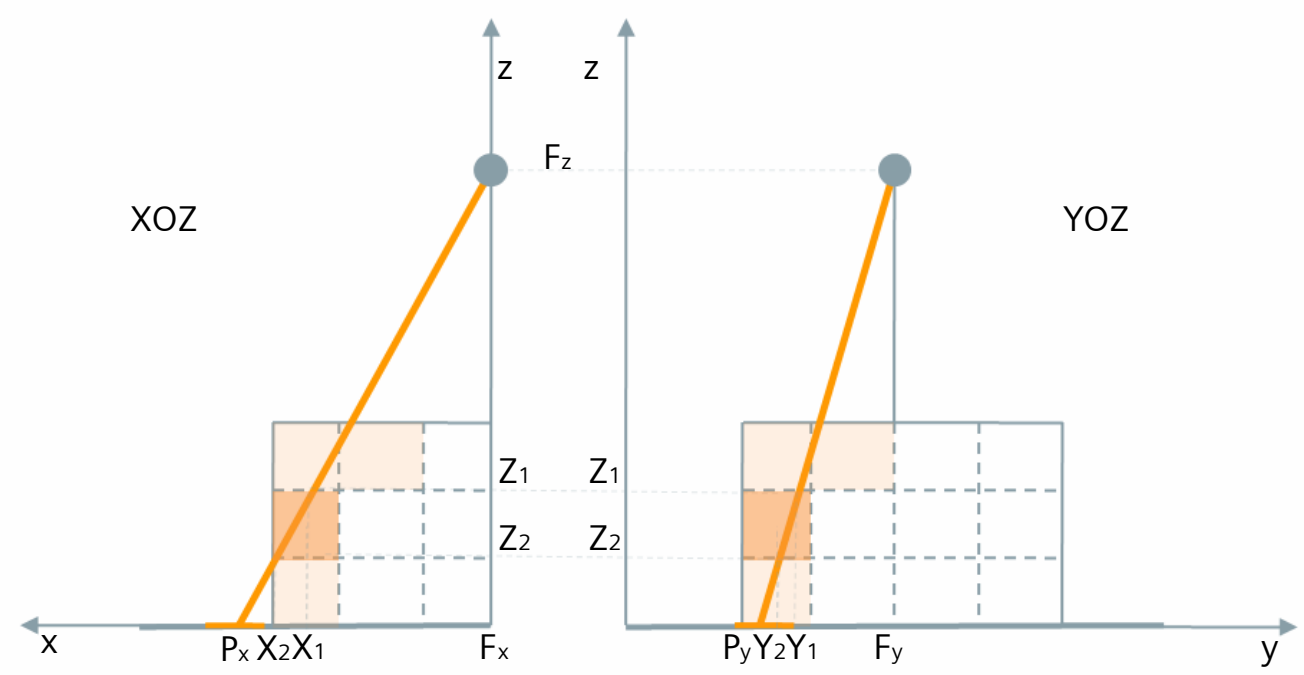

Figure 5.6 Diagram illustrating the projections on the XOZ and $\mathrm{YOZ}$ plans of the 3D image showed in Figure 5.5.

The resultant system matrix, $\mathrm{A}$, is a $2 \mathrm{D} \mathrm{V} \times \mathrm{P}$ matrix for each projection, as equation 5.4 shows.

$$
A_{v p}=\left[\begin{array}{ccc}
A_{11} & \cdots & A_{1 P} \\
\vdots & \ddots & \vdots \\
A_{V 1} & \cdots & A_{V P}
\end{array}\right]
$$

These system matrices reflect the geometry of the tomosynthesis system and the relation between each voxel and each thin beam. The system matrices are used to formulate the back projection step in each reconstruction method. The multiplication of the system matrix by the detected image, $y_{p}$, for each projection, returns an estimate for the volume to be reconstructed.

$$
\left[\begin{array}{lll}
X_{1} & \cdots & X_{V V}
\end{array}\right]=\left[\begin{array}{ccc}
A_{11} & \cdots & A_{1 P} \\
\vdots & \ddots & \vdots \\
A_{V 1} & \cdots & A_{V P}
\end{array}\right] \times\left[\begin{array}{c}
Y_{1} \\
\vdots \\
Y_{P}
\end{array}\right]
$$

The way how these matrixes were constructed eliminates the need of a normalization step related to the length of the ray and its portion inside the voxel. Since each element of these matrixes is a value that corresponds to the fraction of a concrete ray inside a concrete voxel. If we sum all the values corresponding to the same ray in the matrix, 
the result will be 1 . If we consider a ray that reaches the pixel $p$, the sum of the contributions for its attenuation by all voxels in the volume is given by equation 5.6.

$$
\sum_{v=1}^{V} A_{v p}=1
$$

\subsubsection{D Image Reconstruction}

Three different reconstruction methods were chosen to perform the 3D image reconstruction in the simulated data.

These algorithms are representative methods for limited angle tomography and they were selected as they are of different categories. FBP, ML-EM and ART were the preferred algorithms and they are an example of an analytical, a statistical and an iterative method, respectively (Figure 5.7).

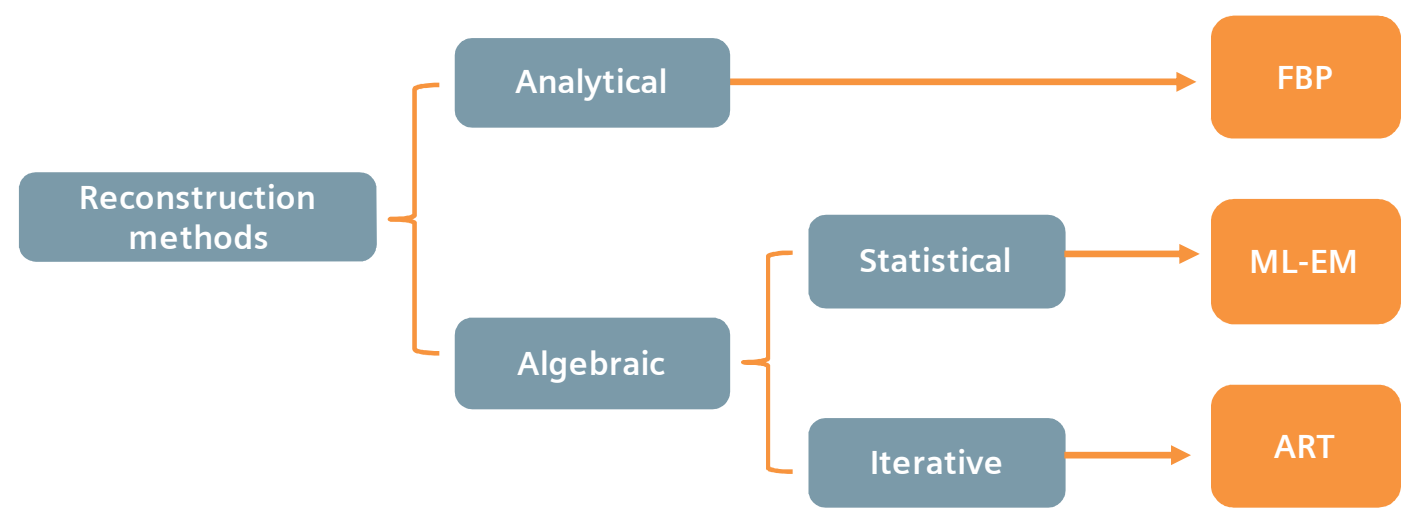

Figure 5.7 Diagram presenting the chosen algorithms, FBP, ML-EM and ART, and theirs categories

The system model previously defined for the breast tomosynthesis is the basis to allow the implementation of these algorithms. Independently of the algorithm, the back projection of a planar image to construct the volume is an important step and it is present in all of them.

The back projection step basically takes the value measured on a pixel of a projection and spreads it along the whole voxels that were crossed by the ray that reached that pixel, accordantly with the probability in the matrix A. This step is repeated successively for each projection. The constructive iteration of all projections will reveal regions of different attenuation coefficients, evidencing different structures of the body. 
The process of back projection can be described by equation 5.6 , where $\hat{\mathrm{X}}_{v}$ is the estimative for the voxel $v$ value, avp is the system matrix value for the combination of the voxel $v$ with the pixel $p$ and $y_{p}$ is the pixel value in the projection data.

$$
\hat{\mathrm{x}}_{\mathrm{v}}=\sum_{\mathrm{p}=1}^{\mathrm{p}} \mathrm{a}_{\mathrm{vp}} \times \mathrm{y}_{\mathrm{p}},
$$

The order how the different projections are analysed by each algorithm can has influence on the final results. This is not the case of the FBP algorithm, as the final volume only results of the sum of back projections. For the remaining algorithms, the order of the views introduced is done in other to maximize the difference between two consecutive introduced views.

To allow the comparison between the different algorithms, the group of projected images was the same used on each one of them. The selected algorithms are discussed in following points.

\subsubsection{Filtered back projection}

The simple back projection algorithm alone presents blurring images. The way to eliminate this artifact is to apply a filter in the measured images. This method, where the back projection is applied to filtered images, is known as filtered back projection algorithm (FBP).

The back projection is still the main step involved in the process of this analytical algorithm. The FBP method can be expressed by equation 5.8, where $\mathrm{H}$ is the filter operator.

$$
\hat{x}_{v}=\sum_{p=1}^{P} a_{v p} \times\left(H \cdot y_{p}\right)
$$

The successive noise addition from the data will be avoided with a previous image filtration. A low-pass filter that uses the neighborhood statistics to estimate the local image value was chosen.

The main steps of the FBP algorithm are explained in the diagram of Figure 5.8. Firstly, the measured images are low-pass filtered. The back projection of these images is 
performed using the system matrix appropriated for each angle. The final volume estimated results from the sum of all back projections.

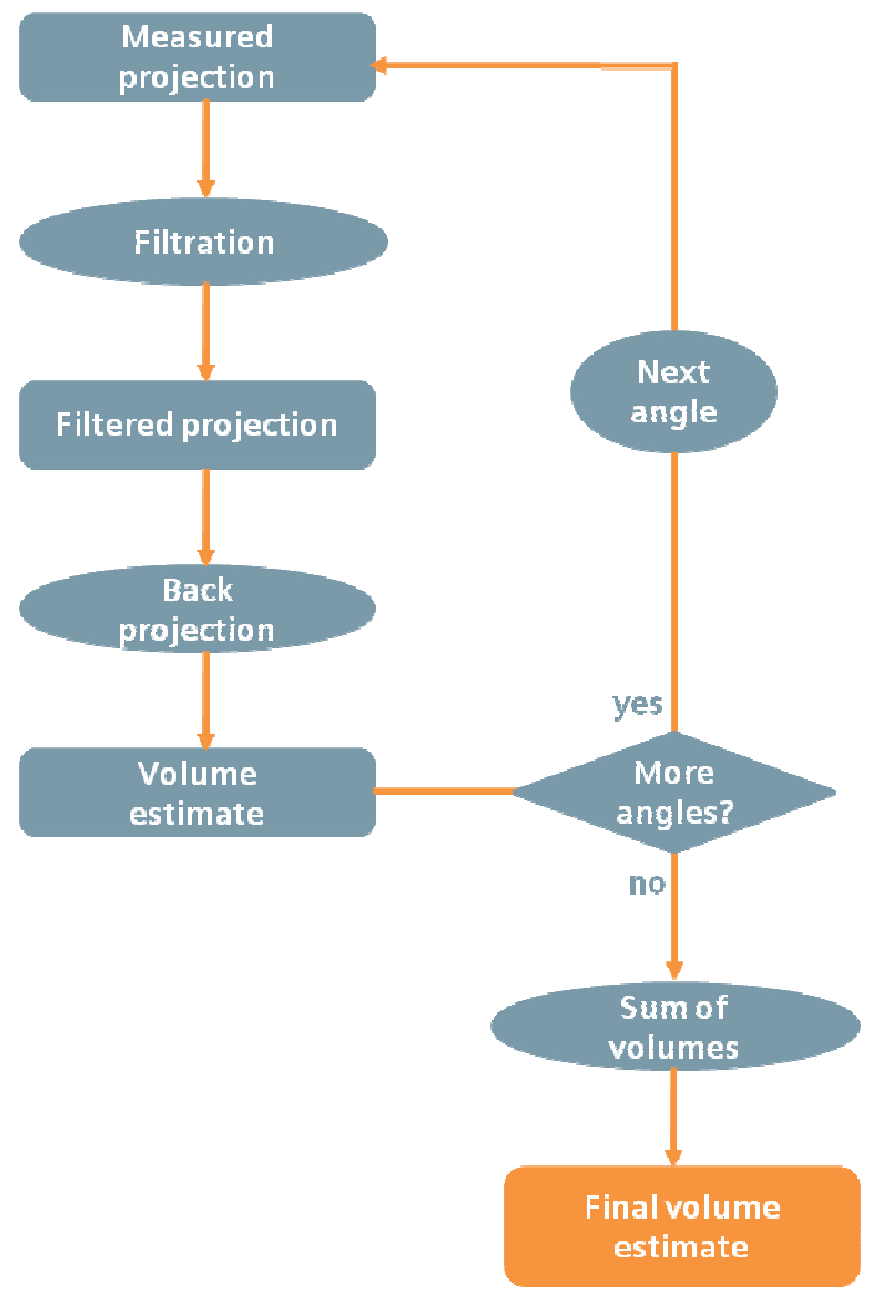

Figure 5.8 Diagram illustrating the principle of the FBP algorithm.

\subsubsection{Algebraic Reconstruction Technique}

The algebraic reconstruction technique (ART) consists of describing the value of each voxel and correct it every iteration, by comparing the measured image with the image estimated from the reconstructed volume of the last iteration, so as to minimize the error between the estimated images and the original measured projections to every point.

Equation 5.9 aims to describe the algebraic reconstruction method. The $\hat{\mathrm{X}}_{v}{ }^{(k+1)}(\mathrm{k}$ is the iteration number and $k=1,2, \ldots)$ is the new estimative for the voxel $v$ value and $\hat{x}_{v}(k)$ is the old estimative for the same voxel. 


$$
\hat{x}_{v}{ }^{(k+1)}=x_{v}{ }^{(k)}+\lambda \times \sum_{p=1}^{p} a_{v p} \times\left(y_{p}-\sum_{v=1}^{v} \frac{x_{v}{ }^{(k)}}{a_{v p}}\right)
$$

$\lambda$ is the relaxation parameter and equal to 0.05 [104]. Other values for $\lambda$, between 0 and 1 , were tested. Values above 0.05 resulted on worst images and values below it did not result on better images.

On the algebraic reconstruction the back projection is done on the comparison between the measured image and an estimative. This estimative initially is a volume where was assigned a value of zero to all its voxels and it is updated every iteration. The error based on the comparison between the last estimative and the original image is then back projected. This new error volume is used to update the last one. This will be projected again until the comparison does not result on the image improvements.

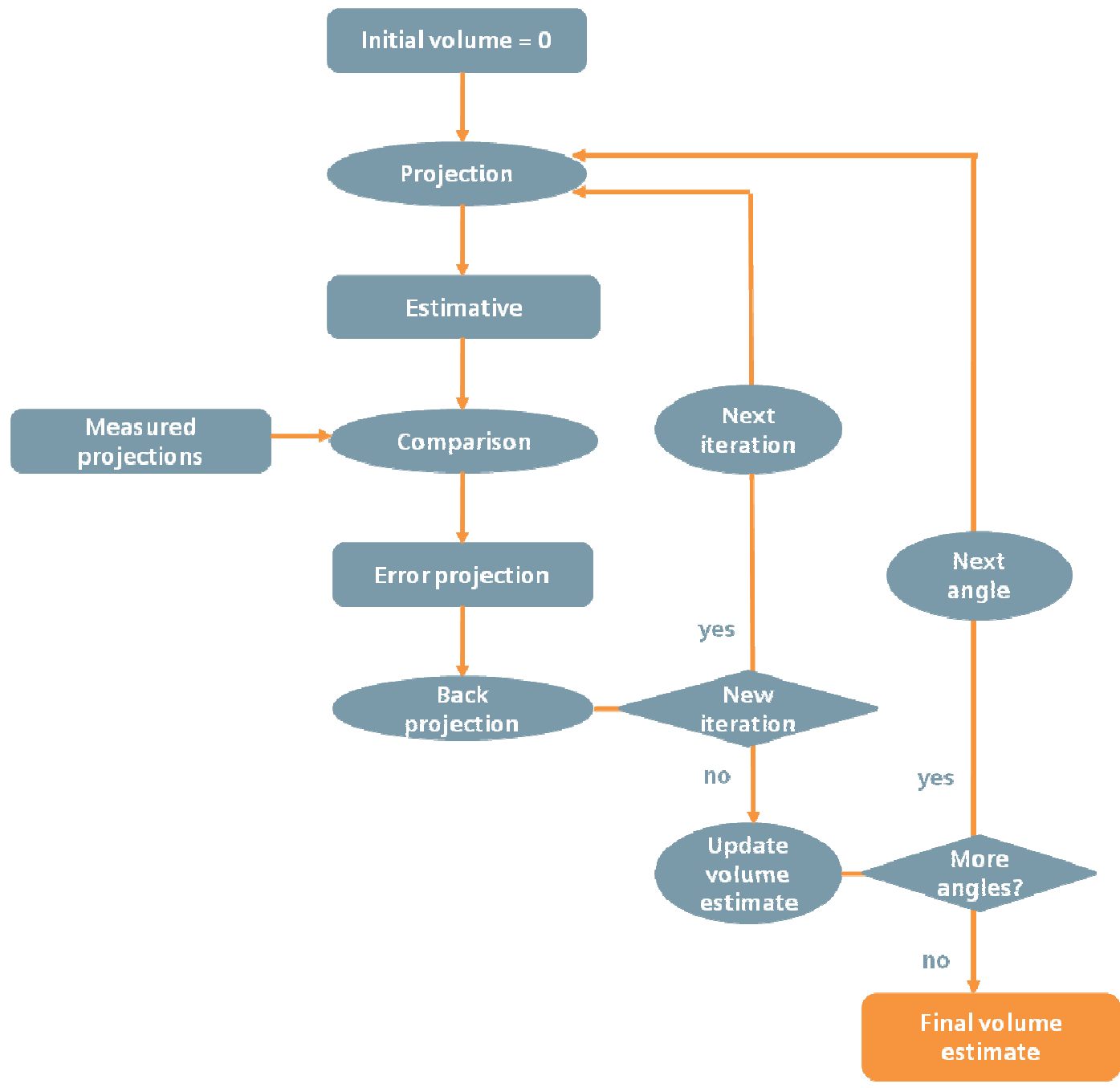

Figure 5.9 Diagram illustrating the steps used to perform the ART algorithm. 


\subsubsection{Maximum-Likelihood Expectation-Maximization}

The maximum-likelihood expectation-maximization (ML-EM) is a statistical algorithm that involves an iterative process to estimate the final values of the reconstructed volume.

The current estimate value for the voxel $v$ results from an update of the last estimate value. This update is done using the ratio between the original data and the forward projection of the last volume estimate. The process comes to a stop when the maximum likelihood solution is found; this is when the estimated volume is the most likely.

$$
\hat{x}_{v}^{(k+1)}=x_{v}^{(k)} \cdot \sum_{p=1}^{p} a_{v p} \times\left(y_{p} / \sum_{v=1}^{v} \frac{x_{v}^{(k)}}{a_{v p}}\right)
$$

The volume estimate begins with a uniform definition for the whole volume. This estimative is updated in each iteration by the multiplication of the last estimative by the back projection of the comparison between the original data and the estimated projection of the last volume.

The steps to perform this reconstruction method and to achieve the volume estimate are described by the diagram in Figure 5.10. 


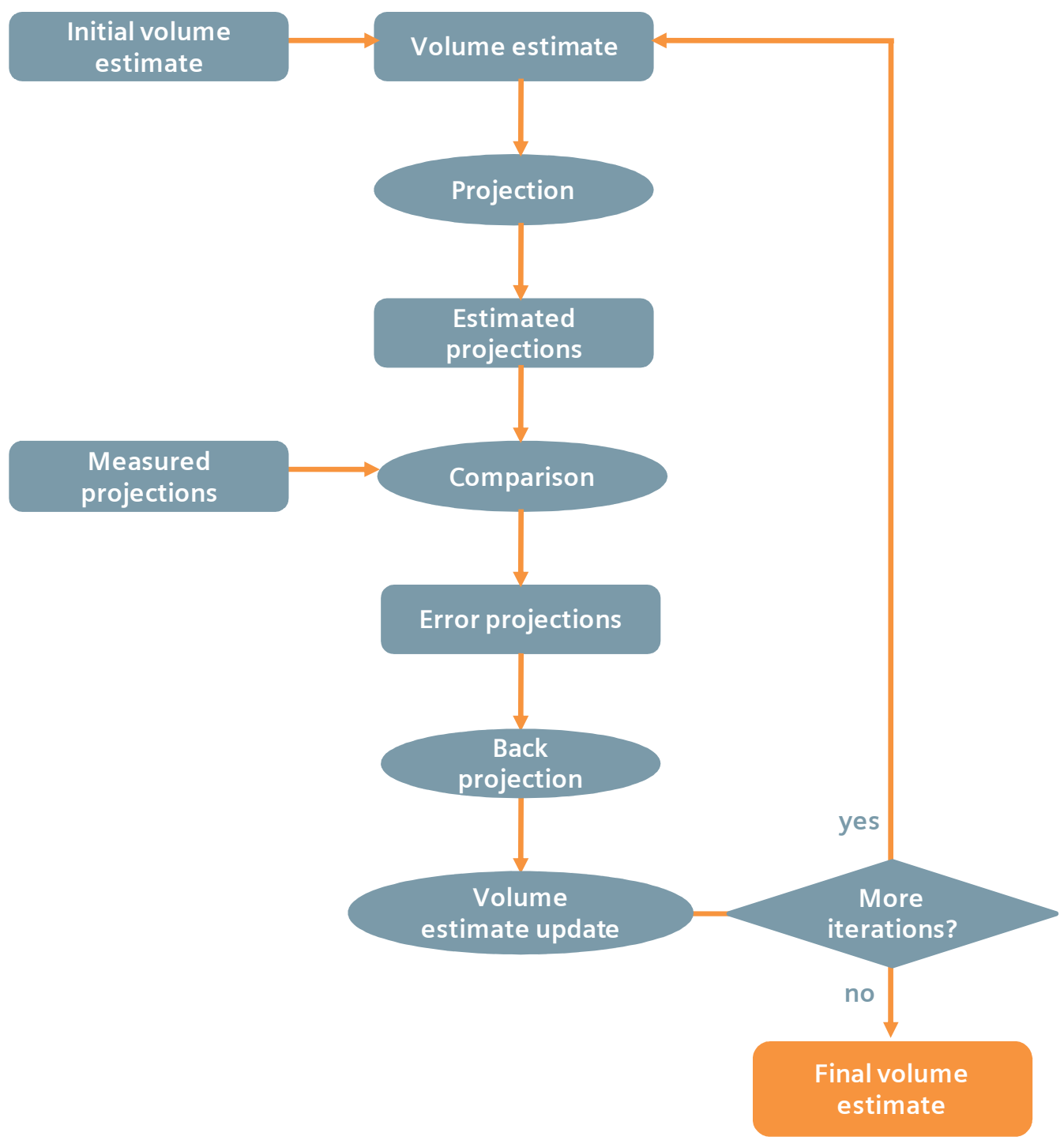

Figure 5.10 Scheme illustrating the principle of the ML-EM algorithm.

\subsection{Image reconstruction evaluation}

To evaluate the algorithm performance different analysis will be done. Besides the visual comparison, the contrast-to-noise ratio (CNR) and the artifact spread function (ASF) will be calculated and the runtime will be measured for each algorithm.

The CNR is important to evaluate the algorithm capability to differentiate the details in the image. The ASF is an indicator used to understand the ability of an algorithm to localize the structures in the slices along the $\mathrm{z}$ axis. The runtime intend the time 
performance evaluation. During the algorithms evaluation, aspects as the blur, the form and appearance of the details and other features will be analysed.

\subsubsection{Contrast-to-noise ratio}

The contrast-to-noise ratio (CNR) allows quantifying the capacity of an algorithm to distinguish the different structures in an image. The calculation of CNR uses the differences of signal between the structure and the image background. The formula used to define CNR is equation 5.11, where Sbg is the background signal, Sobj is the object signal and obg is the root mean square value of the background signal [105].

$$
\mathrm{CNR}=\frac{\mathrm{S}_{\mathrm{bg}}-\mathrm{S}_{\mathrm{obj}}}{\sigma_{\mathrm{bg}}}
$$

The CNR value will be calculated for each algorithm, taking as object the ellipsoid, one of the spheres of $2 \mathrm{~mm}$ of radius and one of the minor cylinders.

A region of $30 \times 30$ pixels (far from the objects) will be used to calculate the Sbg value. $\sigma \mathrm{bg}$ is the root mean square value of this region. Three regions of $4 \times 4$ pixels, $2 \times 2$ pixels and $1 \times 4$ pixels will be used to estimate the Sobj value for the ellipsoid, for the sphere and for the cylinder, respectively. Each signal will be measured in the object plan.

\subsubsection{Artifact spread function}

The image resolution in the thickness direction can be defined by the artifact spread function (ASF). It quantifies the artifact amplitude along the $z$ axis direction. Equation 5.12 is the formula used to calculate the ASF along the $z$ axis. Here Sbg is the background signal, Sartif is the artifact signal, $\mathrm{Sbg}_{\mathrm{g}}\left(\mathrm{zo}_{0}\right)$ is the background signal on the object plan and Sobj(zo) is the object signal on the object plan [102].

$$
\operatorname{ASF}(z)=\frac{S_{b g}(z)-S_{a r t i f}(z)}{S_{b g}\left(z_{o}\right)-S_{o b j}\left(z_{o}\right)}
$$

ASF will be calculated for plans above and below of the ellipsoid plan. This was the object chosen to analyze the ASF because its signal can be well estimated comparing with the smallest objects. A region of $4 \times 4$ pixels inside the ellipsoid (or inside its artifact) will be used to calculate $\mathrm{S}_{\mathrm{obj}}(\mathrm{zo})$ (or Sartif). Another region of $30 \times 30$ pixels (far from the objects) will be used to estimate the Sbg along the successive plans. 


\subsubsection{Runtime}

The fulfilment time is important to evaluate the time performance of the different algorithms. An algorithm can lose its interest if its runtime delays the normal workflow of a service.

The MATLAB functions tic and toc are used to get the precise time required to complete the algorithm. The tic function starts a stopwatch timer and the function toc prints the elapsed time since tic was used. The elapsed time printed is the algorithm runtime.

The workstation where the calculations were done is an Intel Core Duo E8400 @3.00GHz CPU and 1.96GB of RAM memory. 



\section{Simulation: Results \& Discussion}

\subsection{Experiment 1}

On the initial tests done on GATE, the systems were created on a simple manner to facilitate the familiarization with this tool. One of those tests, aimed to understand the influence of the materials in the photon counting, using geometric phantoms.

The result obtained in this test is illustrated in Figure 6.1. The figure shows the number of photons that reaches the detector matrix with the phantom described in Table 4.2, under the conditions also described in that table.

The influence of the material density variation is visible by the photons counting, as expected. In the image, the smaller cylinder of silicon is visible within the larger cylinder made of breast material. This is related to the number of photons that are attenuated by breast and silicon material. The attenuation depends on the thickness of tissue, as well as its linear attenuation coefficient and its density. The density of the breast material used is $1.020 \mathrm{~g} / \mathrm{cm}^{3}$, while the density of silicon is $2.33 \mathrm{~g} / \mathrm{cm}^{3}$. 


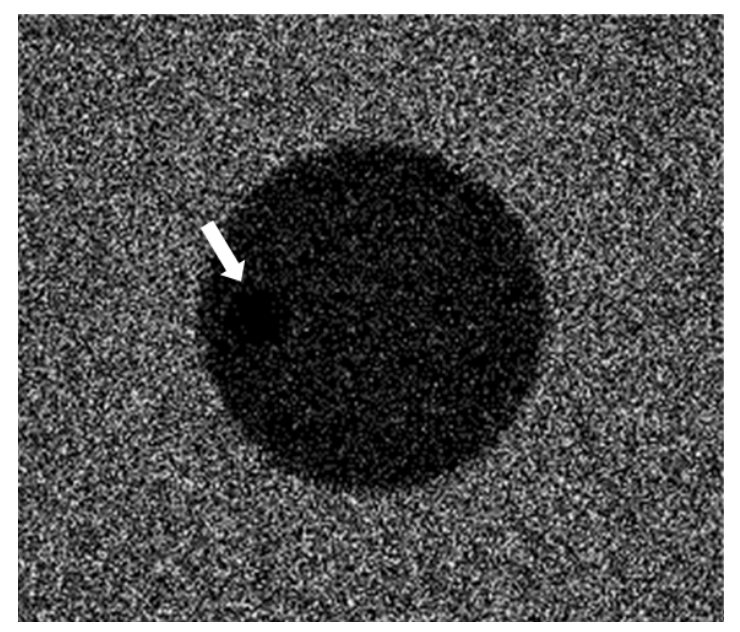

Figure 6.1 Result obtained by the cylinder phantom. The figure shows the number of photons that reaches the detector (black is the null counting and white represents a detected photon).

\subsection{Experiment 2}

On a more elaborated simulation, another phantom was used (Figure 4.5). The cylindrical phantom used has various details, since the simulated images will be used to perform the 3D image reconstruction. The architecture of this system is described in Table 4.3. The acquisition was done around an arc of 50 degrees each 5 degrees, in a total of 11 images taken. The results of this model are shown in Figure 6.2.

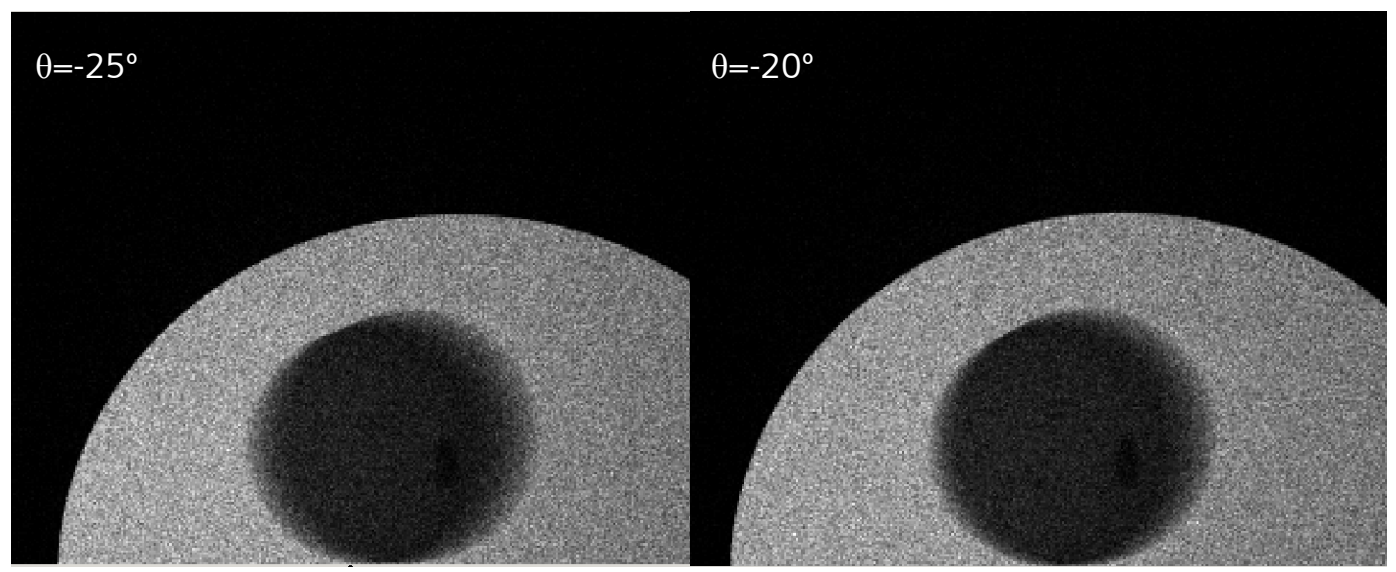



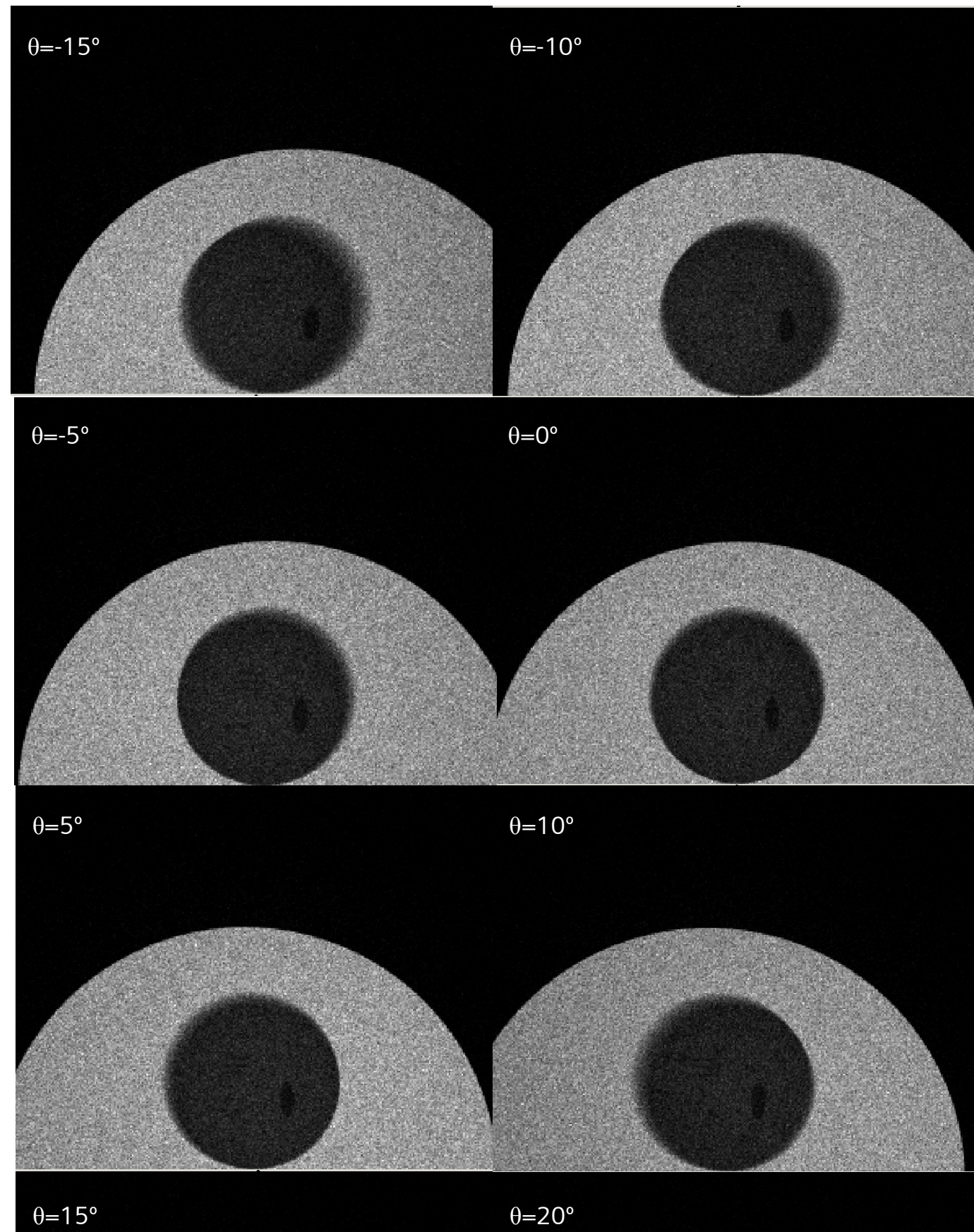


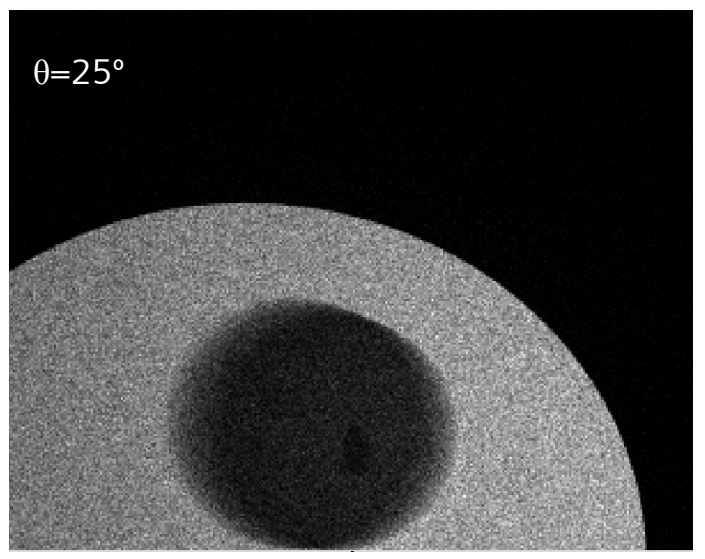

Figure 6.2 Results of the simulation using the model described in Table 4.3. The 11 images are taken each 5 degrees performing an arc of 50 degrees.

Each image matrix is a 2D matrix, composed by $300 \times 240$ pixels of $1 \times 1$ millimetres.

\subsection{Experiment 3}

In the previous experiments, only analytical phantoms were used, but realistic phantoms are also important. One of the experiments included a more realistic phantom, the NCAT phantom. The breast volume was cropped from de phantom body and it was introduced on GATE as a voxellized phantom. The system was defined as it demonstrated in Table 4.5 and the results are presented in Figure 6.3.

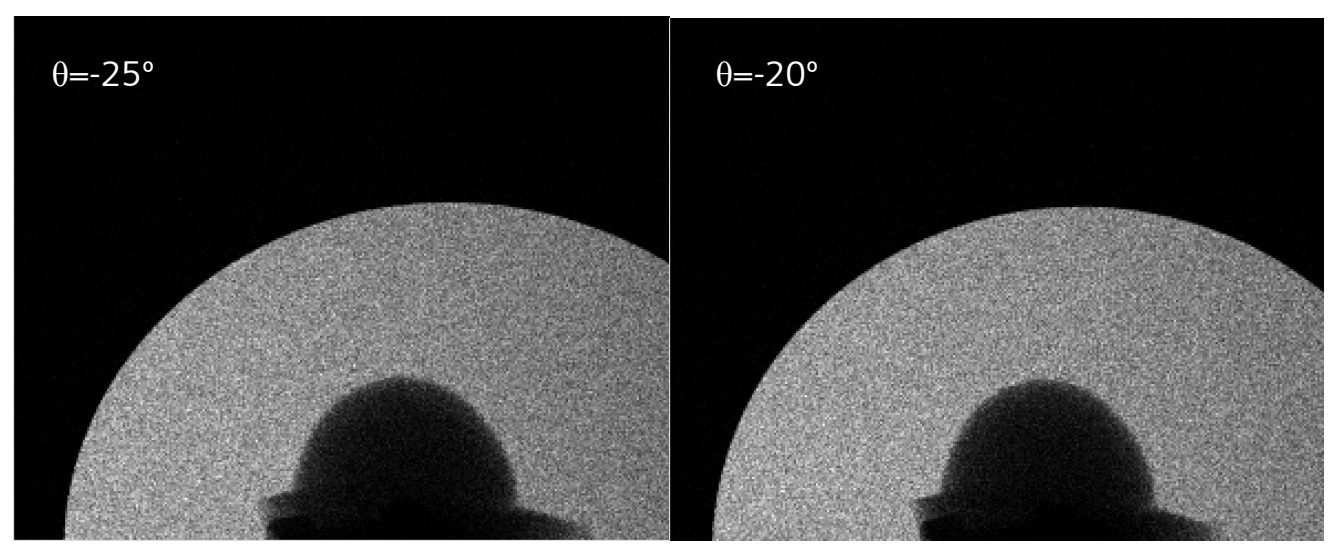




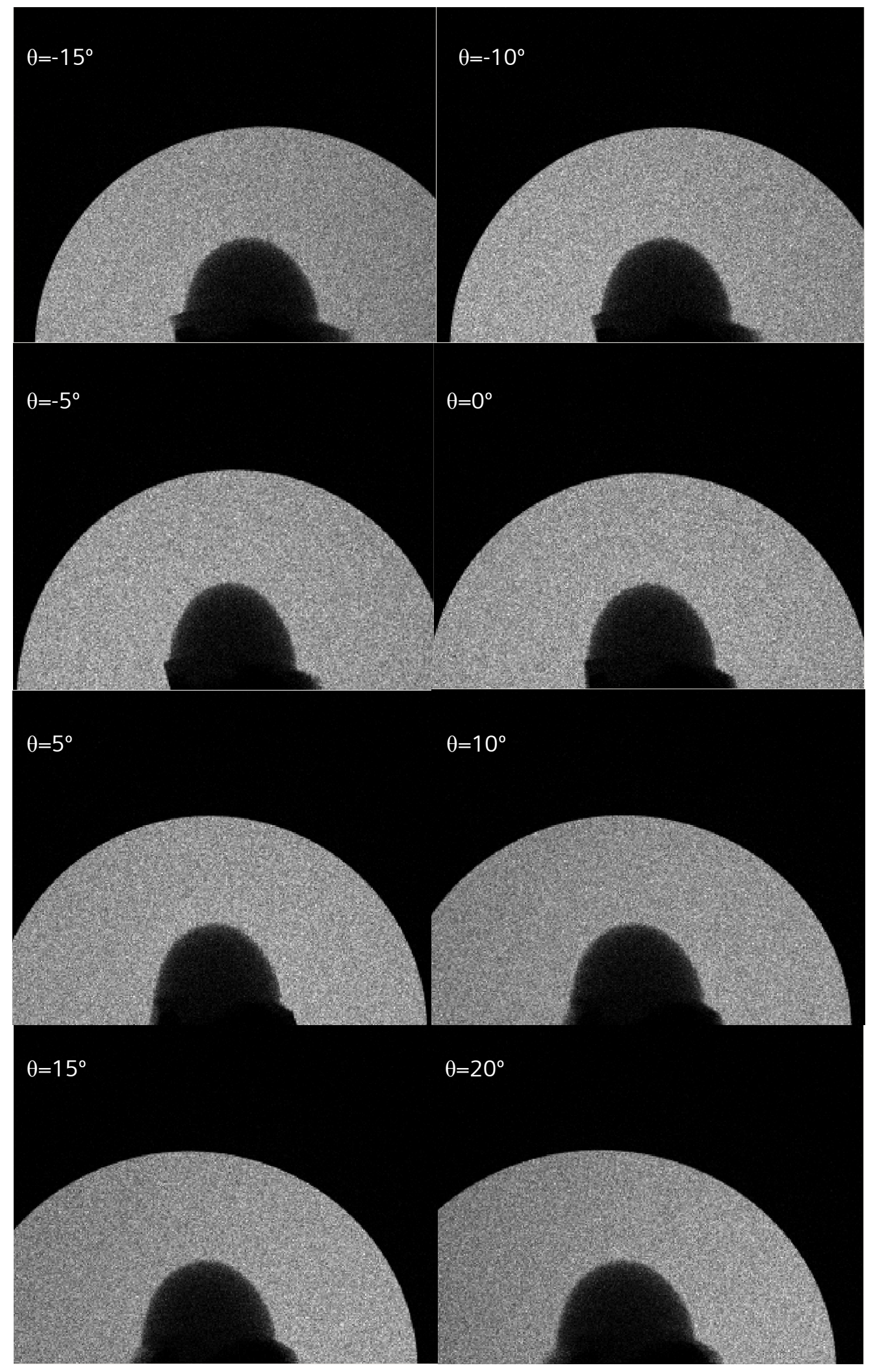




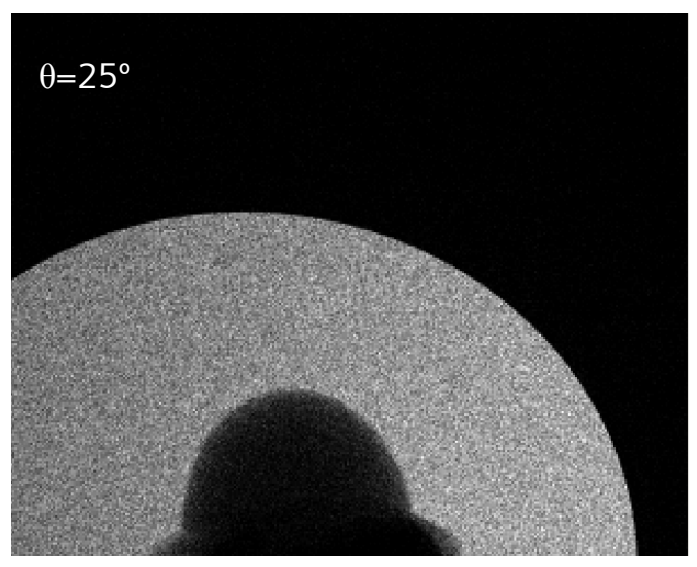

Figure 6.3 Simulated data using the NCAT breast phantom. 11 projections were taken around an arc of 50 degrees each 5 degrees.

AS the last experiment, each image matrix is a 2D matrix, composed by $300 \times 240$ pixels of $1 \times 1$ millimetres.

\subsection{Discussion}

The experiment 1 was one of the earliest tests done on GATE. The result was presented on the first intercalary presentation of this project and also on the published article (Annex 1).

The idea was to understand how to define the x-ray beam used in the mammographic exam, how to define the volumes and its characteristics and to know the influence of the materials on the resultant photon counting. The initial experiments were very important to the familiarization with GATE and to explore the existent resources available in the program: the materials database; the options for the output; the different forms to define the phantoms.

By observing the Figure 6.1, it is interesting to note the influence of the silicon material in the photon counting. This simple simulation shows how that material can decrease the image quality and affect the diagnostic quality, using projection images.

The objective of the experiment 2 was to acquire images for the 3D reconstruction step and that was successfully completed. Figure 6.2 presents these images. Different details were introduced in the phantom to create structures which allow the comparison between the original volume and the same volume after the 3D reconstruction. These details create variations of the material density and consequently changes on the 
photon attenuation. The different materials and theirs different forms and size allow a better analysis of the 3D image reconstruction.

In the simulated data, the ellipsoid is perfectly discriminated from the breast material that defines the major phantom. The cylinders can also be visible from the background, although not so easily. Moreover, the material that defines the cylinders (Silicon) is denser than the material that defines the ellipsoid and the spheres (Weddellite). Although the reasonable results for the bigger details, it is difficult to differentiate the smaller ones, the spheres, from the other structures. Their dimensions allied to the density of the material do not allow theirs distinction. Moreover, the smallest spheres have $1 \mathrm{~mm}$ of radius and the detector resolution is $1 \times 1 \mathrm{~mm}$. This can be an important point to the comparison between these projected data and the reconstructed volume.

In the experiment 3, a more realistic phantom was used to simulate the mammographic acquisition. In the images present in Figure 6.3, variations in the photon counting can be perceived. The difference in the photon attenuation is a consequence of the phantom thickness that varies in different parts. It also a result of the differences in the tissues that describe the whole volume, since breast tissue, bone, lungs and air are part of the region selected.

These results are more realistic than the analytical phantom. Although, the resultant images from the realistic phantom use, should only be employed to perform the reconstruction step after the algorithm assessment that must be performed using analytical phantom. Hence, the results were not used to perform the reconstruction step, during the present work. 



\section{Reconstruction: Results \& Discussion}

The simulated images of the analytical phantom were used to perform the image reconstruction. The goal is to reconstruct the whole phantom volume from the projected images obtained by Monte Carlo simulations. To perform it, FBP, ART and MLEM were the methods used.

The results obtained for each reconstruction method were different from each other. The resultant volume is presented in the following points and the algorithm performance analysis is done after that.

In the reconstruction process, the whole volume estimated has $240 \times 300 \times 50$ voxels of $1 \times 1 \times 1$ millimetres. The volume is shown on slices of $240 \times 300 \times 1$ voxels, but not all of the 50 slices will be presented, for sake of simplification. 26 slices were selected either because they are in or near of plan of one of the objects, or because they show a significant difference between the last slice selected. Nevertheless, the selected slices are the same for the three algorithms, to allow the comparison between them.

The plan with the lowest $z$ value is the nearest plan from the detector. Oppositely, the plan with the maximum value for $z$ is the plan farthest from the detector and nearest from the source. 


\subsection{Filtered Back Projection Results}

The 26 transversal slices of the estimated volume resultant from the FBP process are shown in Figure 7.1.

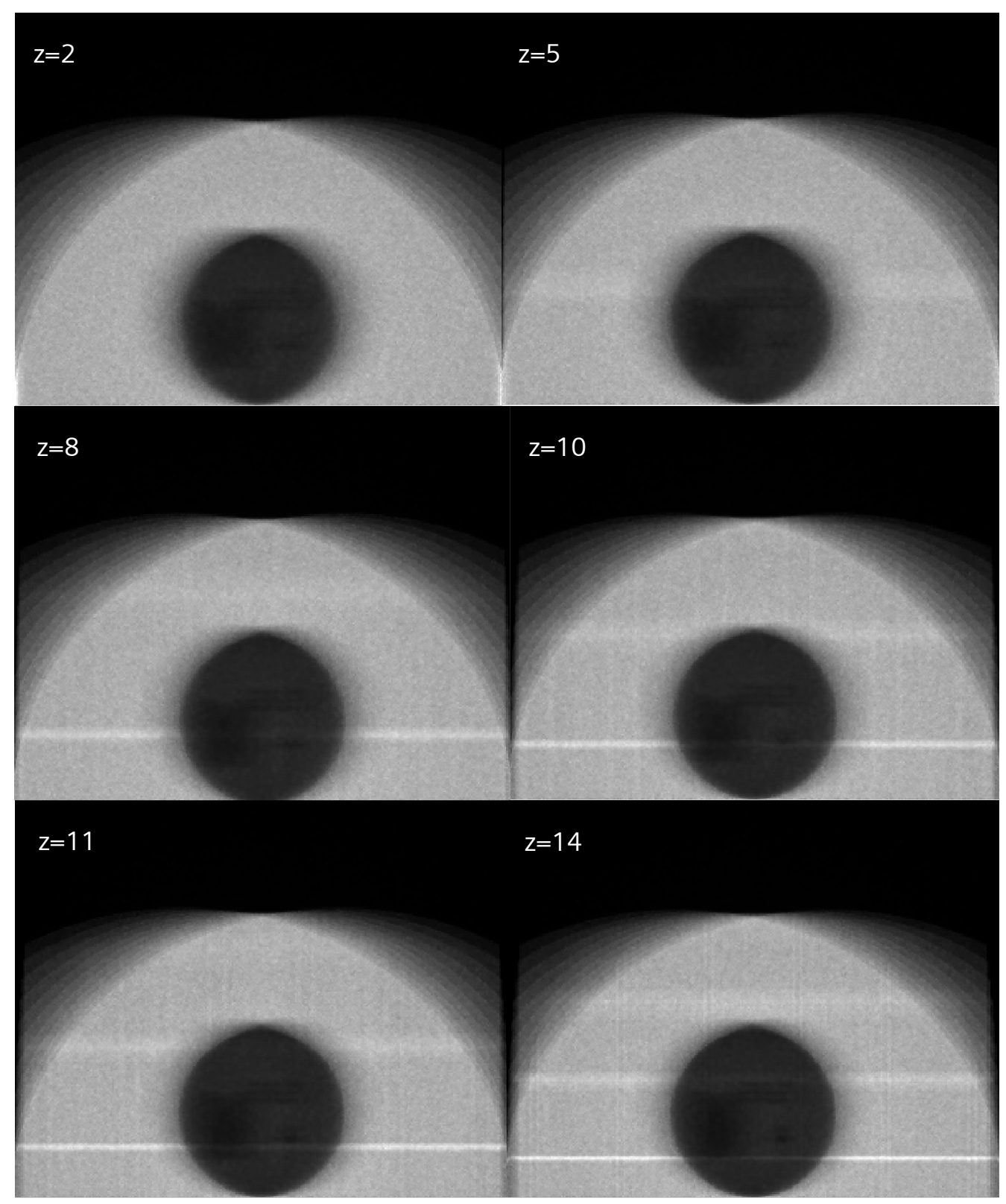




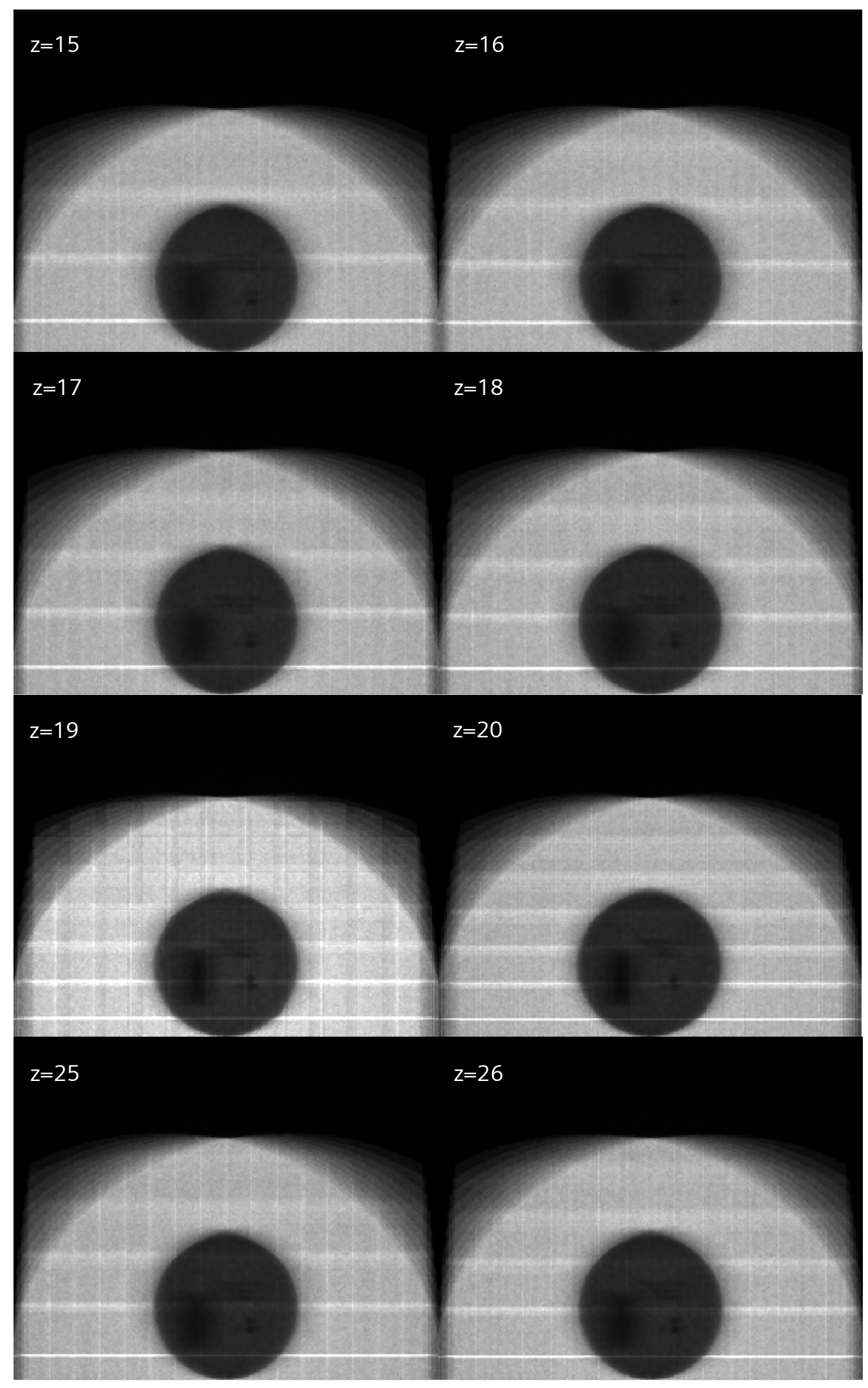




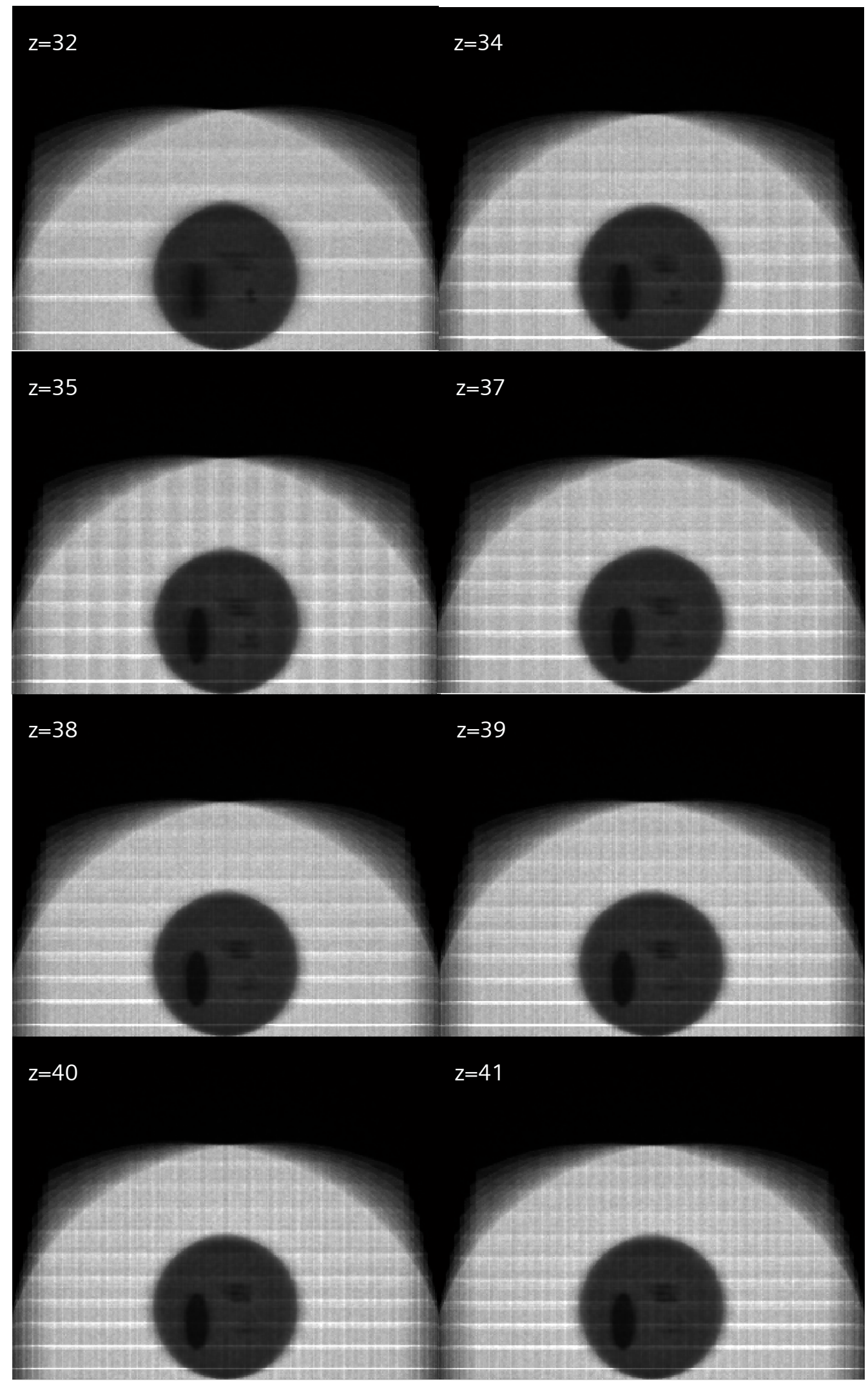




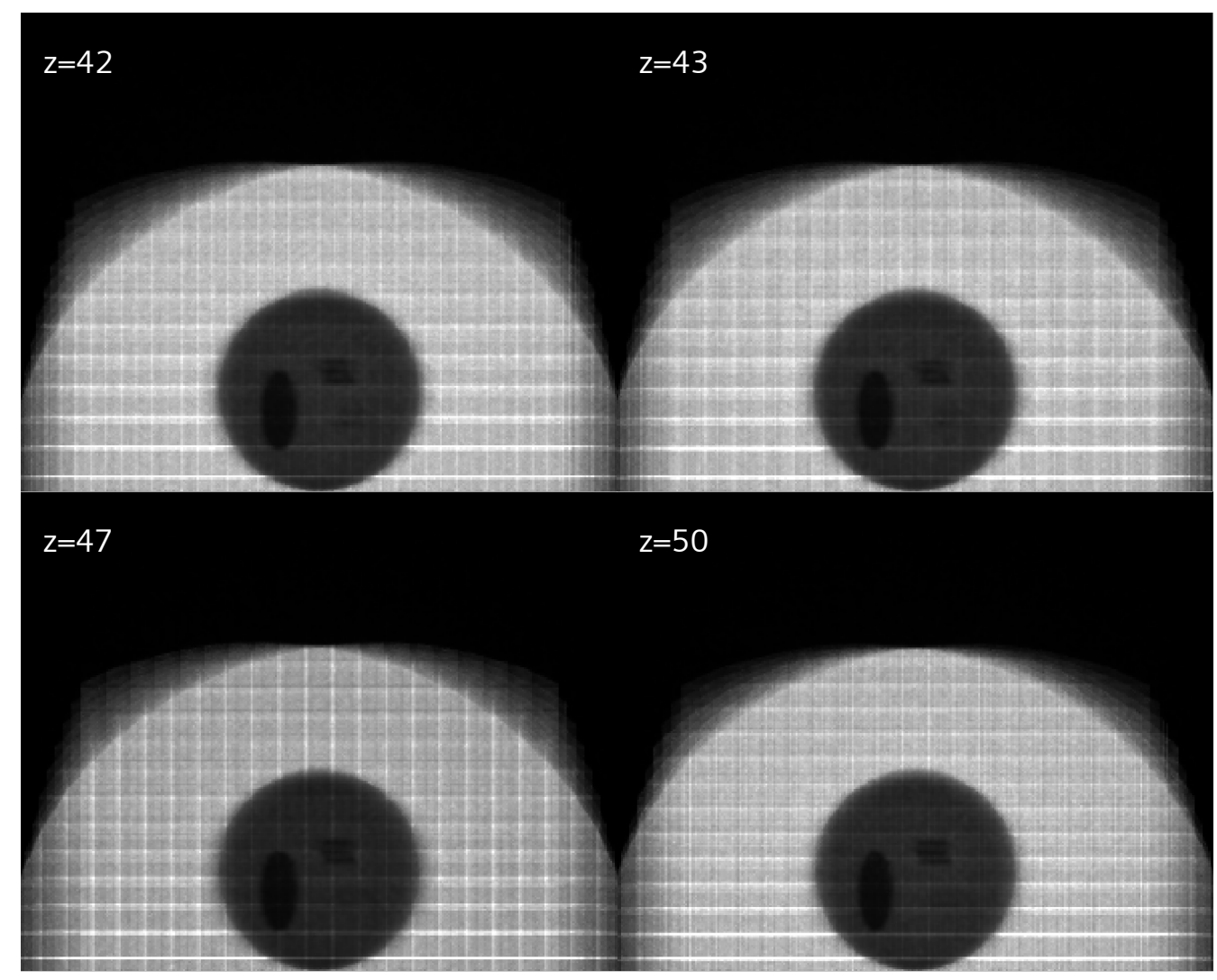

Figure 7.126 of the 50 slices of the volume estimated by the FBP reconstruction method.

Using an appropriated program to the image visualization, the whole volume can be displayed as it can be seen in Figure 7.2. This way of visualization allows the successive sampling of the different slices on different plans (transversal, sagittal and coronal plans). Once encountered a region of interest, it can easily be located in the 3D space. Pointing crosshairs in the region, its localization on the three plans is showed. Figure 7.2 illustrates this procedure to localize one of the spheres of the phantom. 


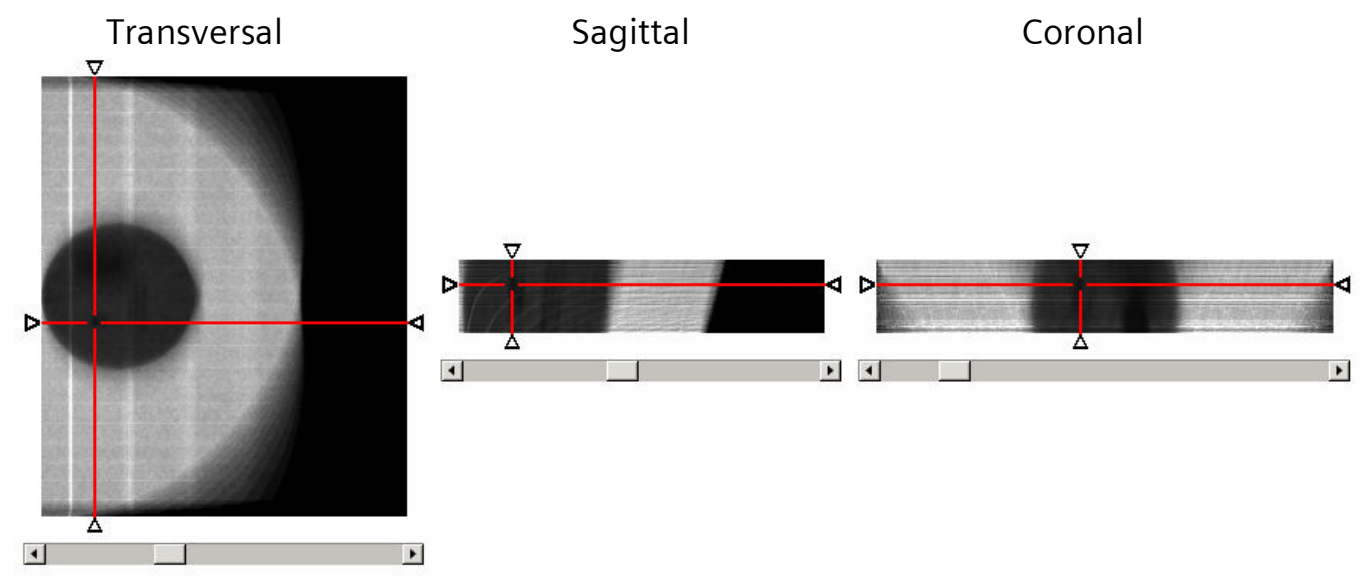

Figure 7.2 Localization on the space of a region of interest selected, using crosshairs on the resultant volume.

\subsection{Algebraic Reconstruction Technique Results}

The volume resultant from the ART procedure is showed in 26 of its 50 slices, in Figure 7.3. The method was implemented accordantly with the diagram presented in Figure 5.9 , with 3 iterations.

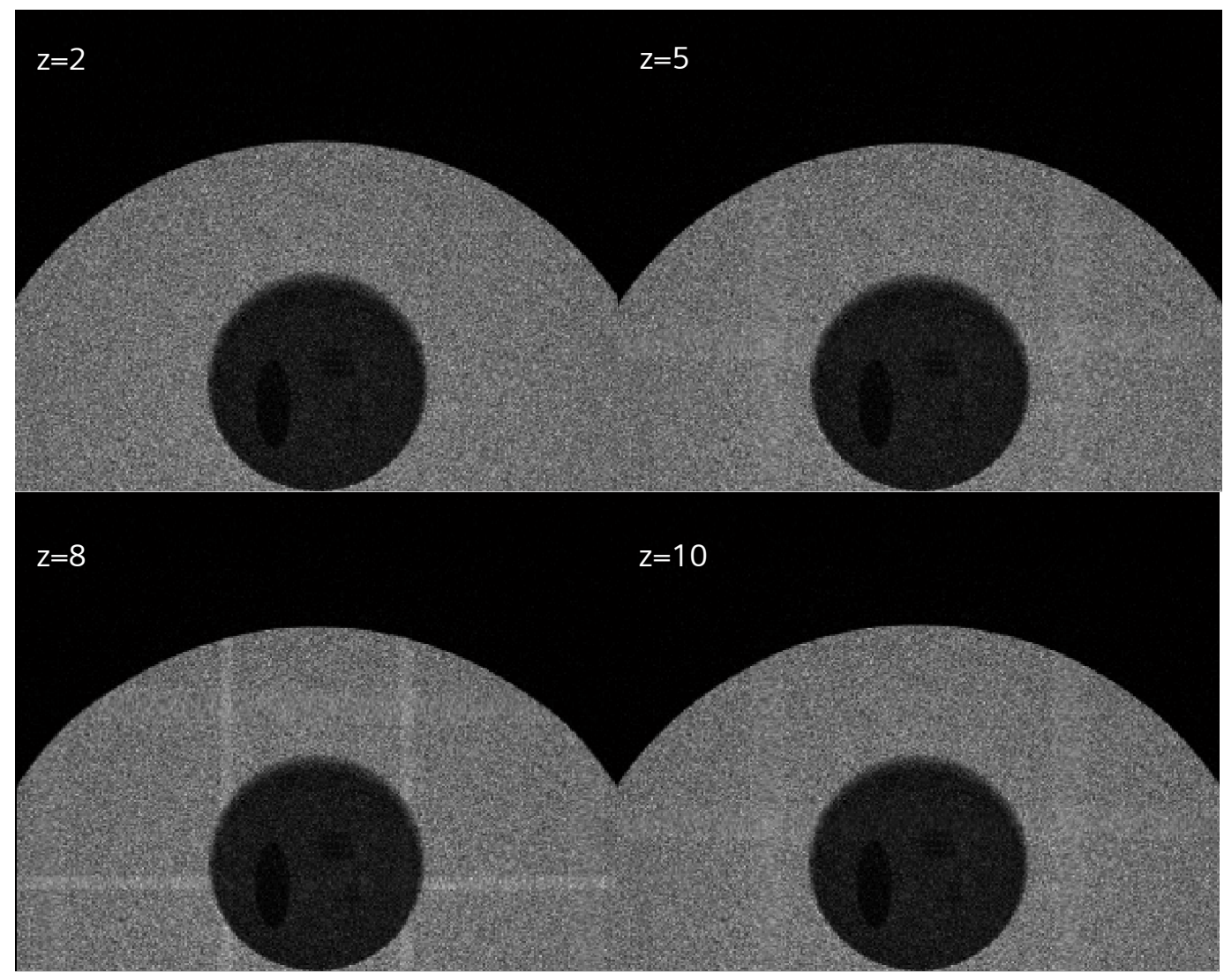




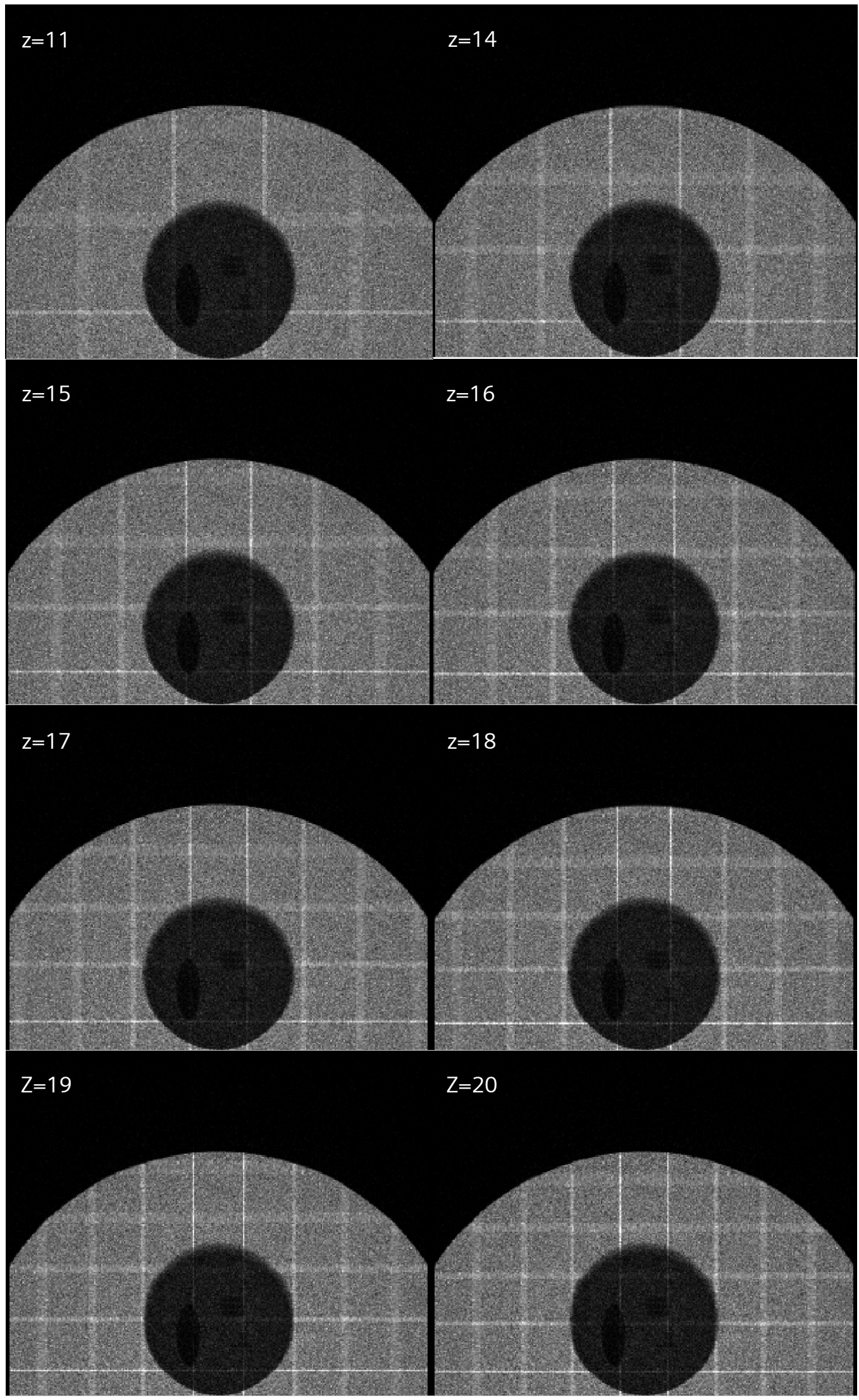




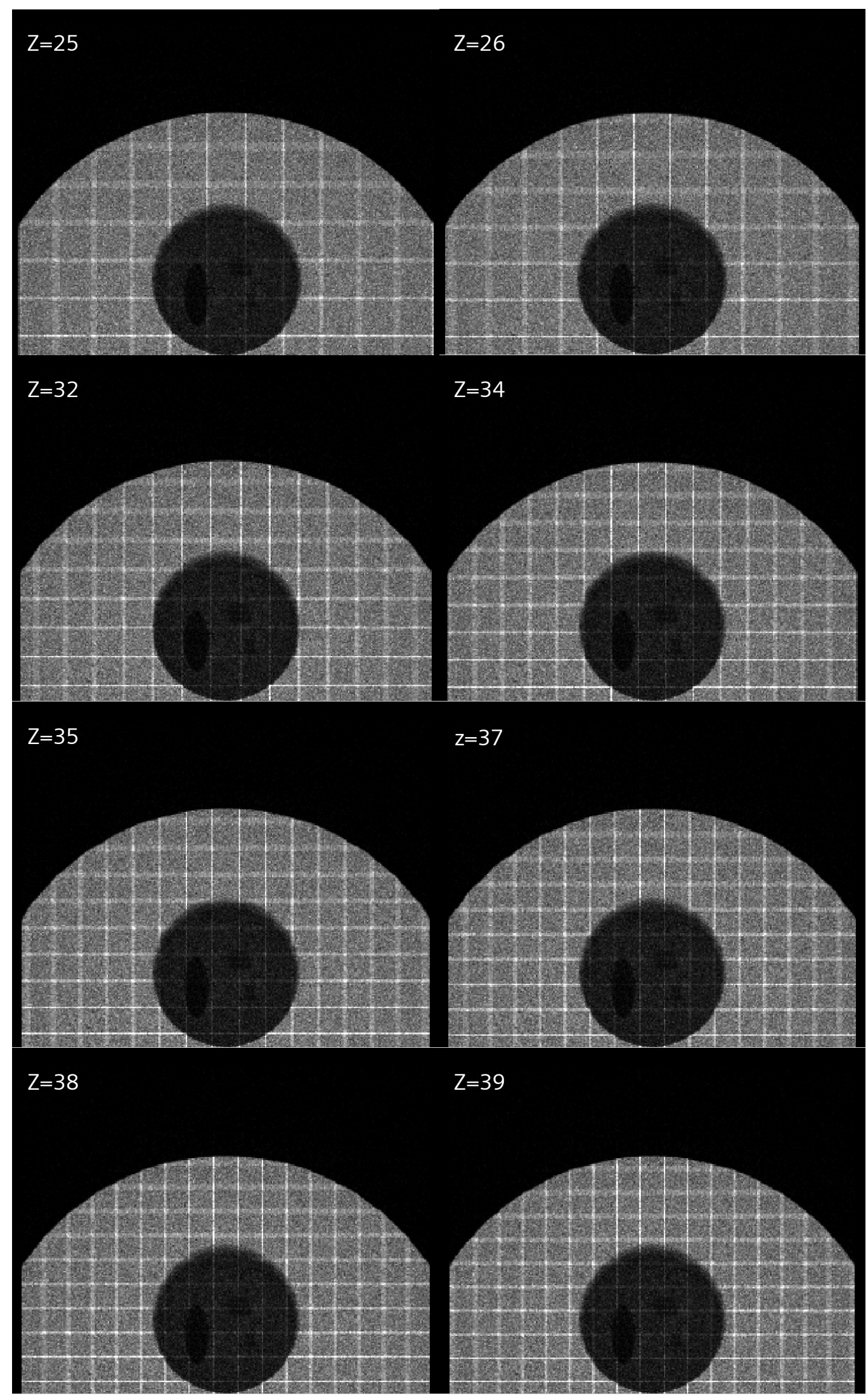




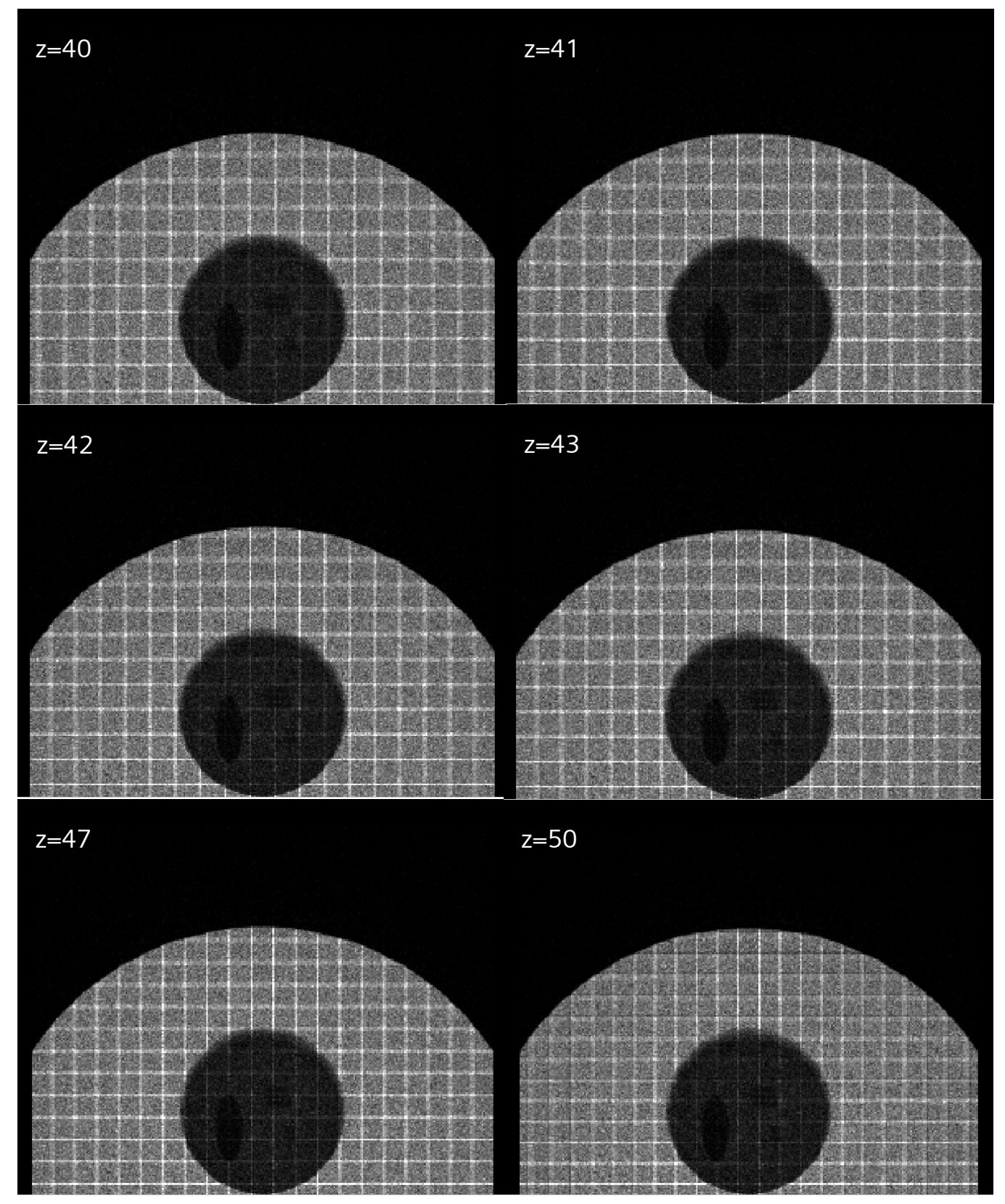

Figure 7.3 Slices of the volume reconstructed by the ART algorithm.

The same procedure made with the FBP was done for the resultant volume of the ART. Figure 7.4 shows the possibility to select a region of interest and locate it on the space. 


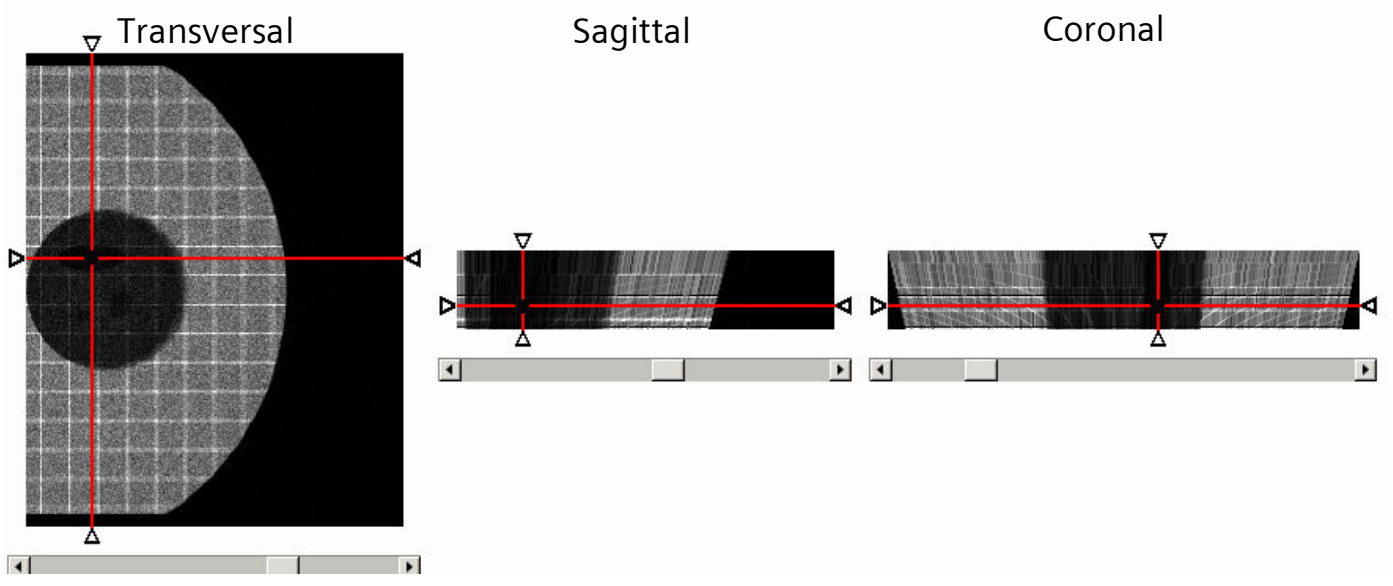

Figure 7.4 Localization on the space of the mass, using crosshairs on the resultant volume.

\subsection{Maximum-Likelihood Expectation-Maximization Results}

After the 3D image reconstruction was applied to the simulated data, using the ML-EM method, the resultant volume can be seen in 26 of its 50 slices, in Figure 7.5. These images are the product of the iterative method described by Figure 5.10, completing 3 iterations.

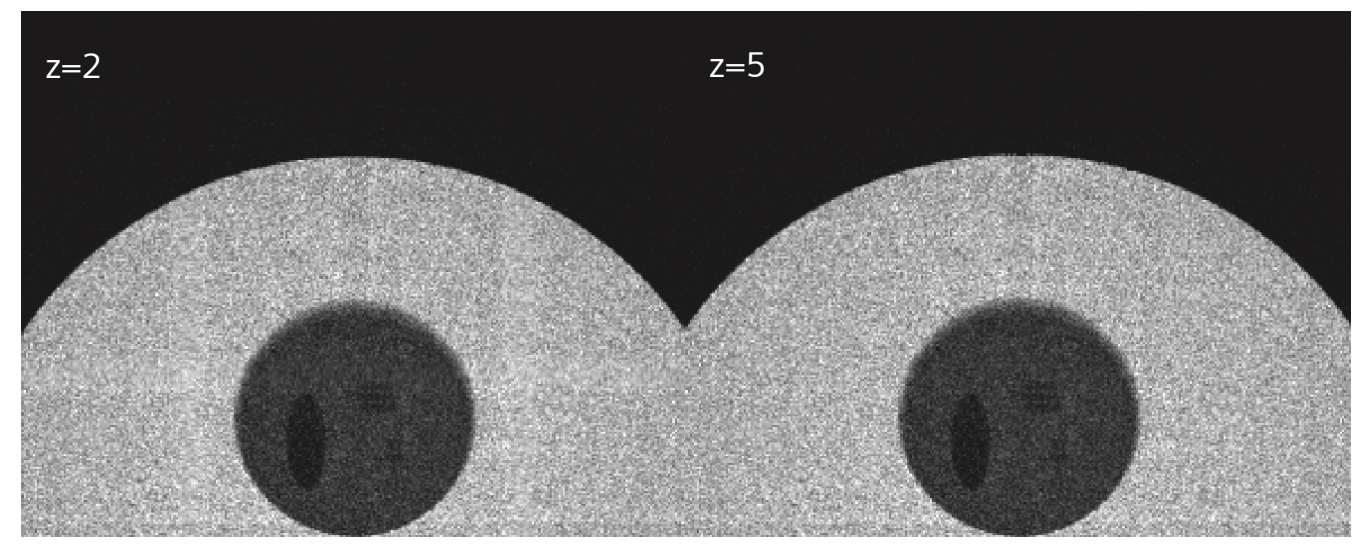



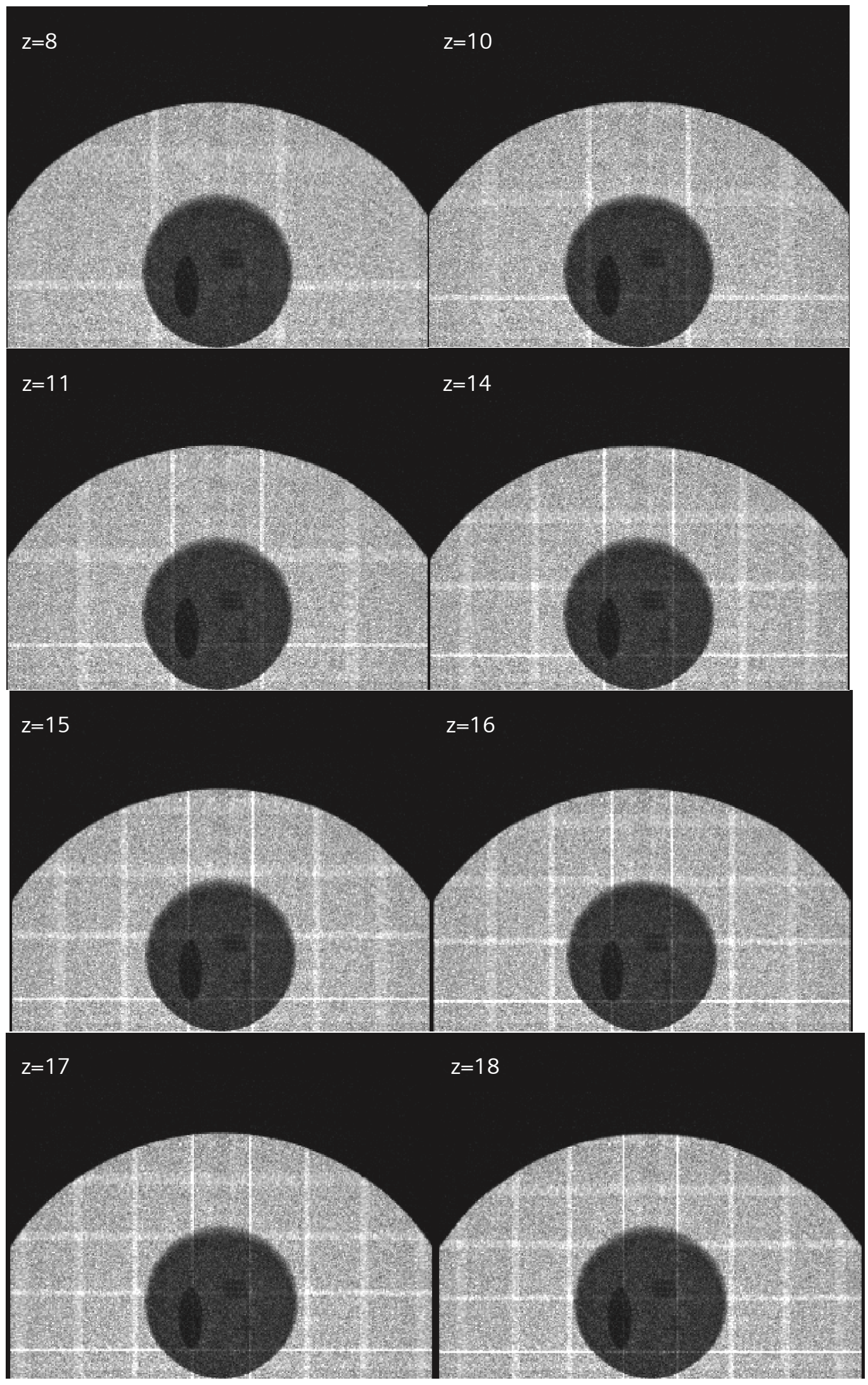


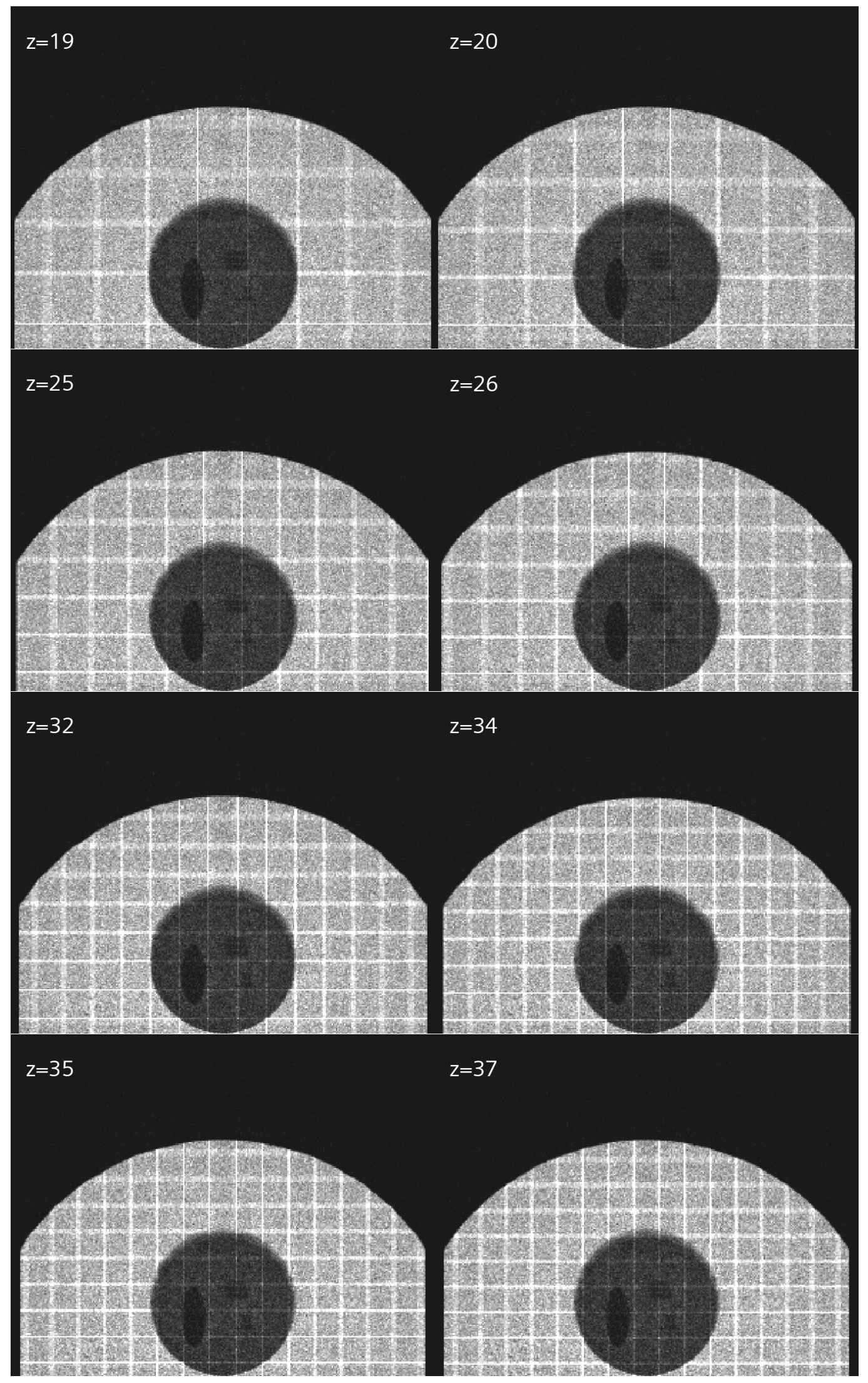




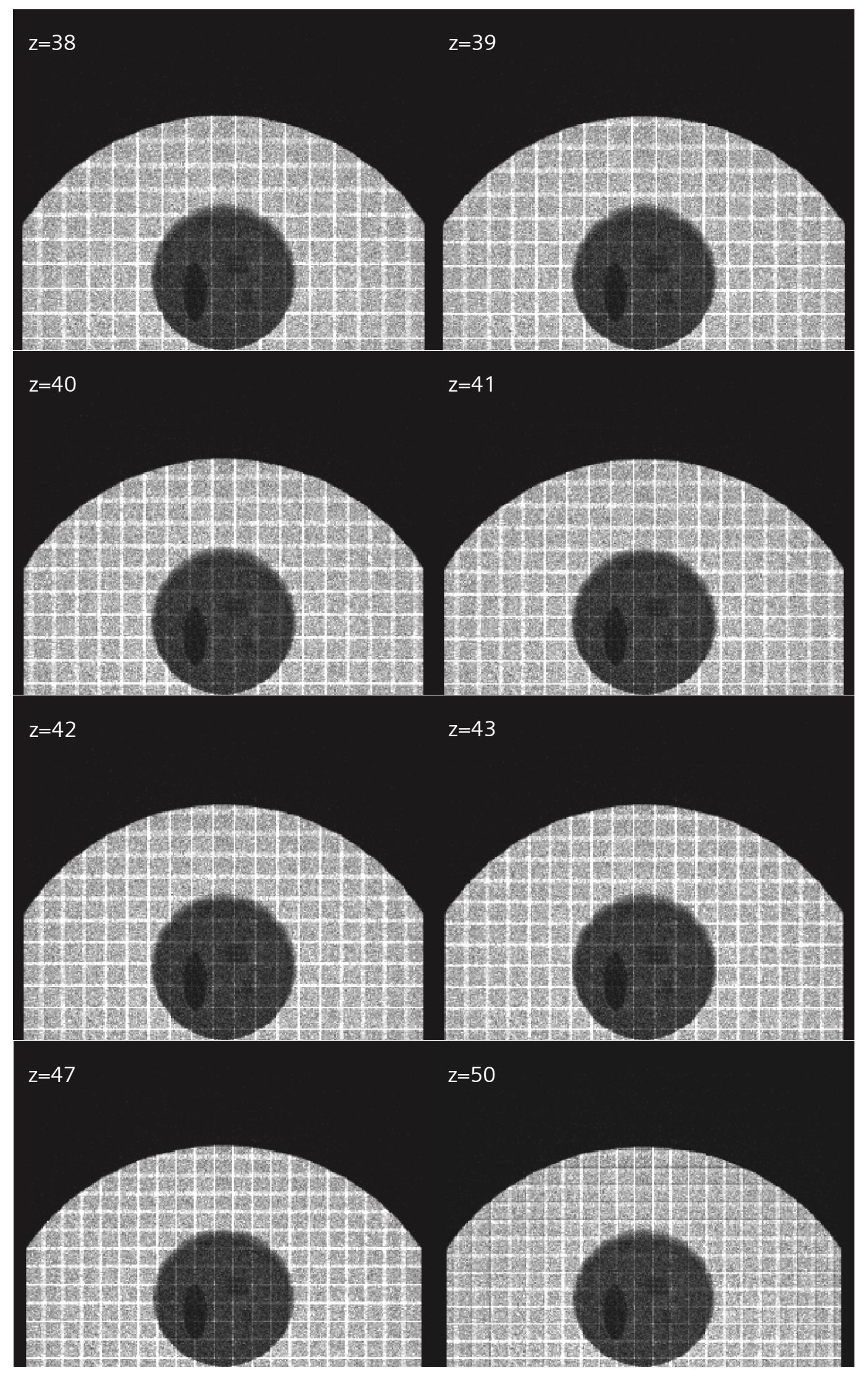

Figure 7.5 The 26 selected slices of the volume reconstructed by the ML-EM method. 
The volume showed in Figure 7.5 can also be seen on slices in the transversal, sagittal and coronal plans. The selection of a region of interest can be observed in Figure 7.6.

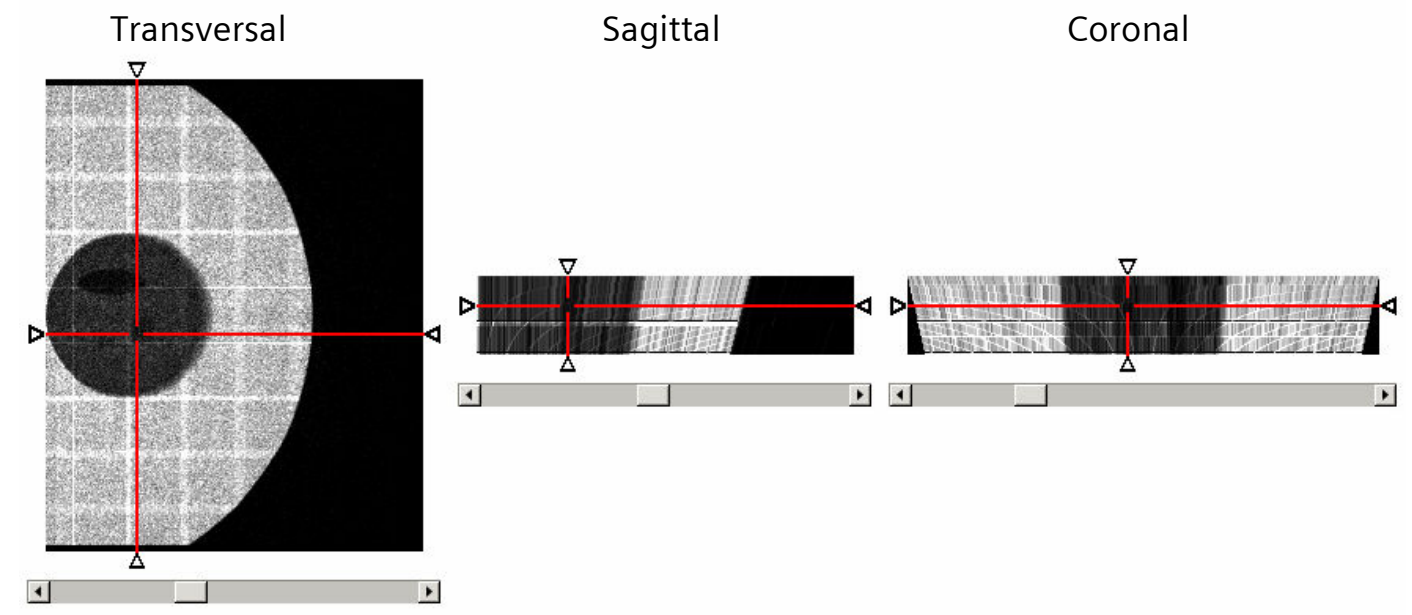

Figure 7.6 Volume visualization on the transversal, sagittal and coronal plans.

\subsection{Figures of Merit}

The indicators described in section 5.4 were calculated to estimate the algorithm performance and to allow the comparison between methods.

The CNR is important to evaluate the algorithm capability to differentiate the details in the image. The ASF is an indicator that reflects the ability of an algorithm to localize the structures in the plans along the $z$ axis. ASF is an important pointer in the case of Digital Breast Tomosynthesis, as the 3D localization is a central point. The runtime intend the time performance evaluation of the different reconstruction methods. During the visual comparison, aspects as the blur, the structures form and appearance and other features will be analysed. In this section, the results for each one are indicated.

The contrast-to-noise ratio was calculated basing in equation 5.11 and using one of each of the different structures inside the phantom: the ellipsoid, a sphere and a cylinder. The results of CNR are presented in Table 7.1. 
Table 7.1 Contrast-to-noise ratio of the three algorithms, calculated for the ellipsoid, sphere and cylinder regions.

\begin{tabular}{|l|l|l|l|}
\hline \multicolumn{4}{|c|}{ Contrast-to-noise ratio } \\
\hline & FBP & ART & ML-EM \\
\hline ellipsoid & 0.691 & 0.626 & 0.624 \\
\hline sphere (2 mm) & 0.052 & 0.198 & 0.179 \\
\hline cylinder & 0.288 & 0.509 & 0.515 \\
\hline
\end{tabular}

To get the perception of these CNR values, the visualization of the same object reconstructed by three different forms is showed in Table 7.2. The greater the contrast is, the better the distinction between structures.

Table 7.2 Comparison between the object visualization on its localization layer.

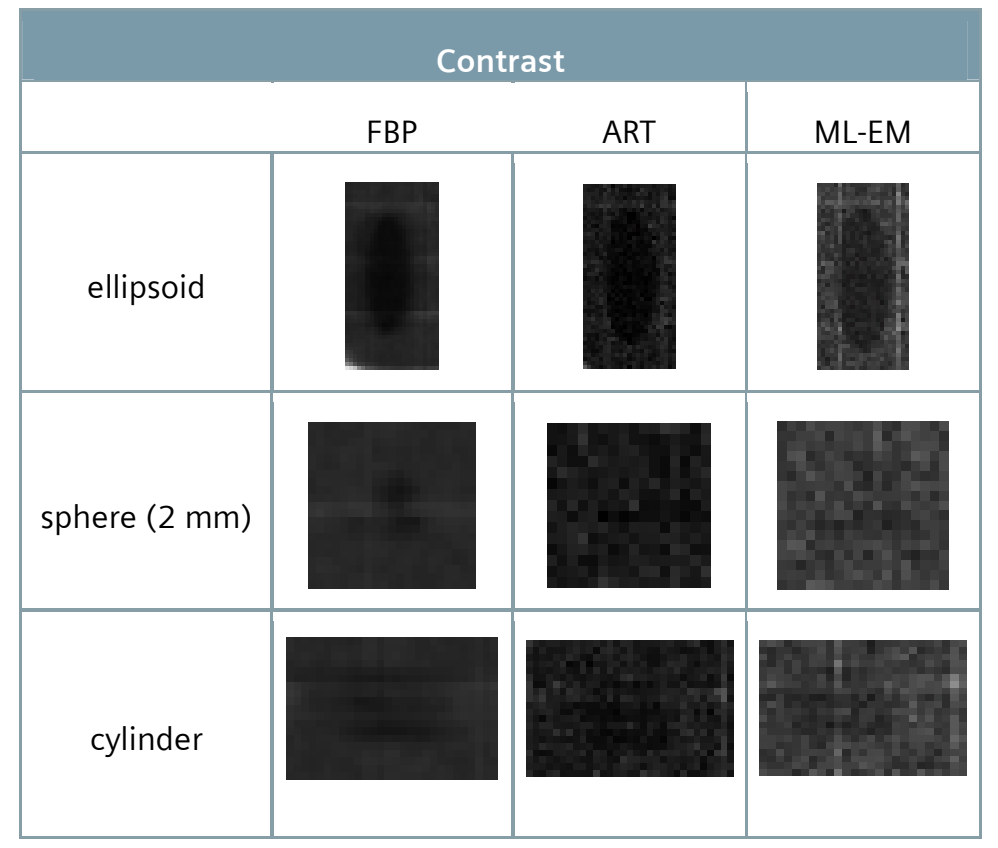

The artifact spread function describes the artifact extension along the $z$ axis. The comparison between the three algorithms is showed for the three types of structures, ellipsoid, sphere and cylinder, in Figure 7.7, Figure 7.8 and Figure 7.9, respectively. The ASF measurement was done using the values from the plans above and below of the object plan, each 2 millimetres and each 1 millimetres for the plans nearest to the object plan. The ideal algorithm should present the value 1 to the ASF for all the plans 
where the structure really is, and the value 0 to the remaining plans. This would mean that the structure would only be seen on the slices where it is.

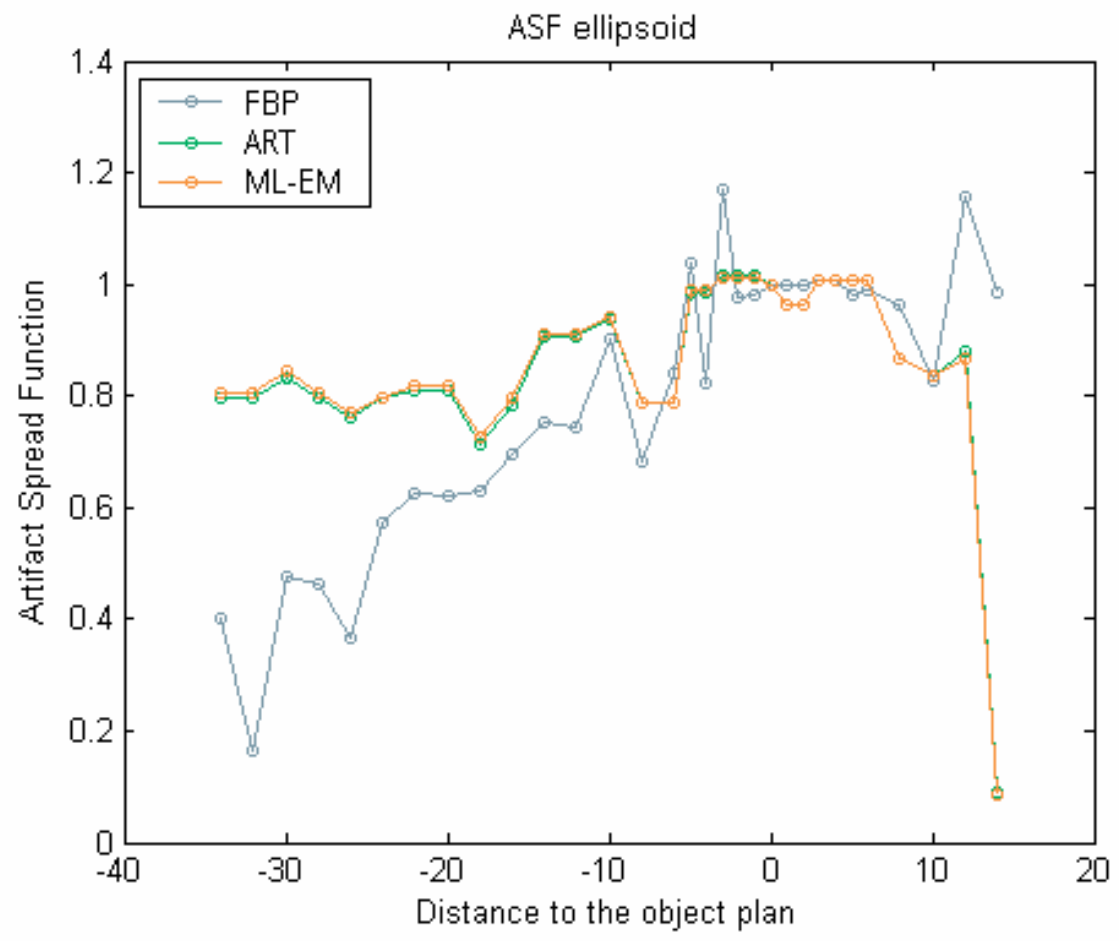

Figure 7.7 Artifact spread function of the ellipsoid object for the three algorithms. 


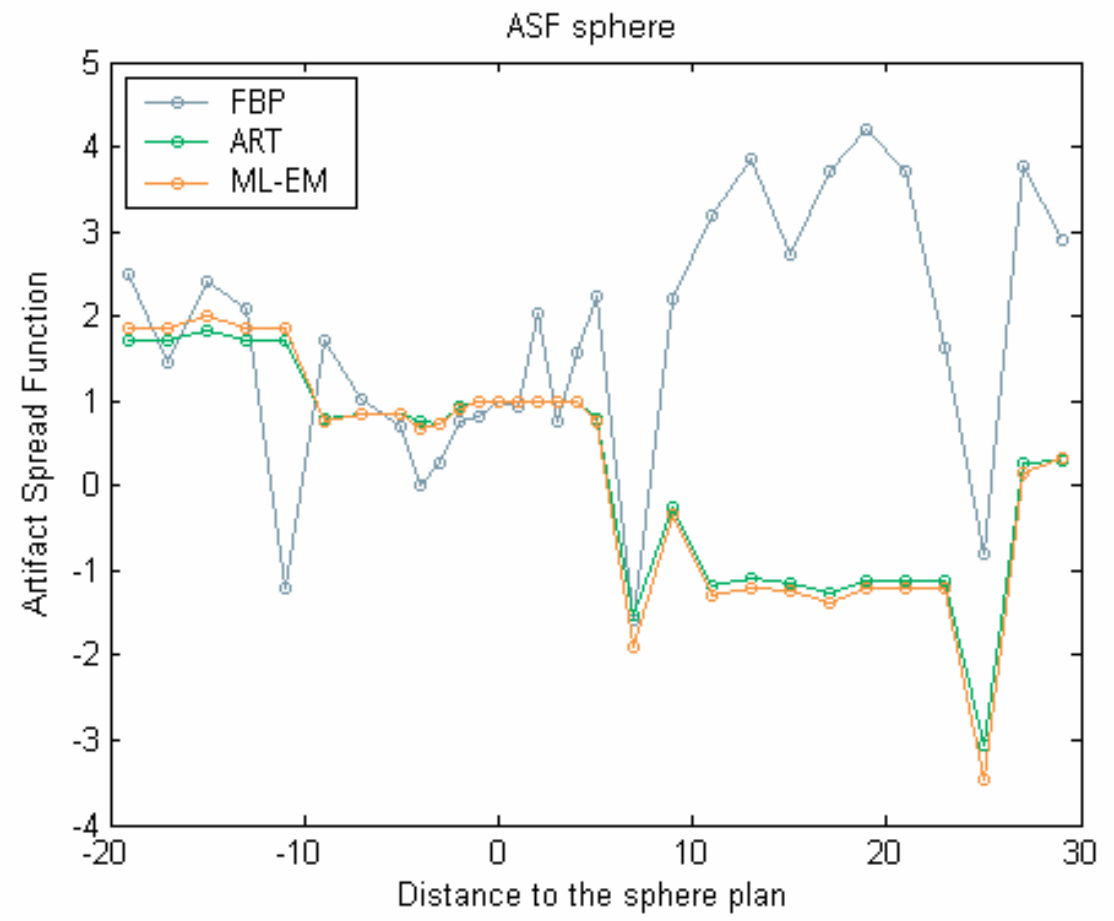

Figure 7.8 Artifact spread function of the sphere (of $2 \mathrm{~mm}$ ) object for the three algorithms.

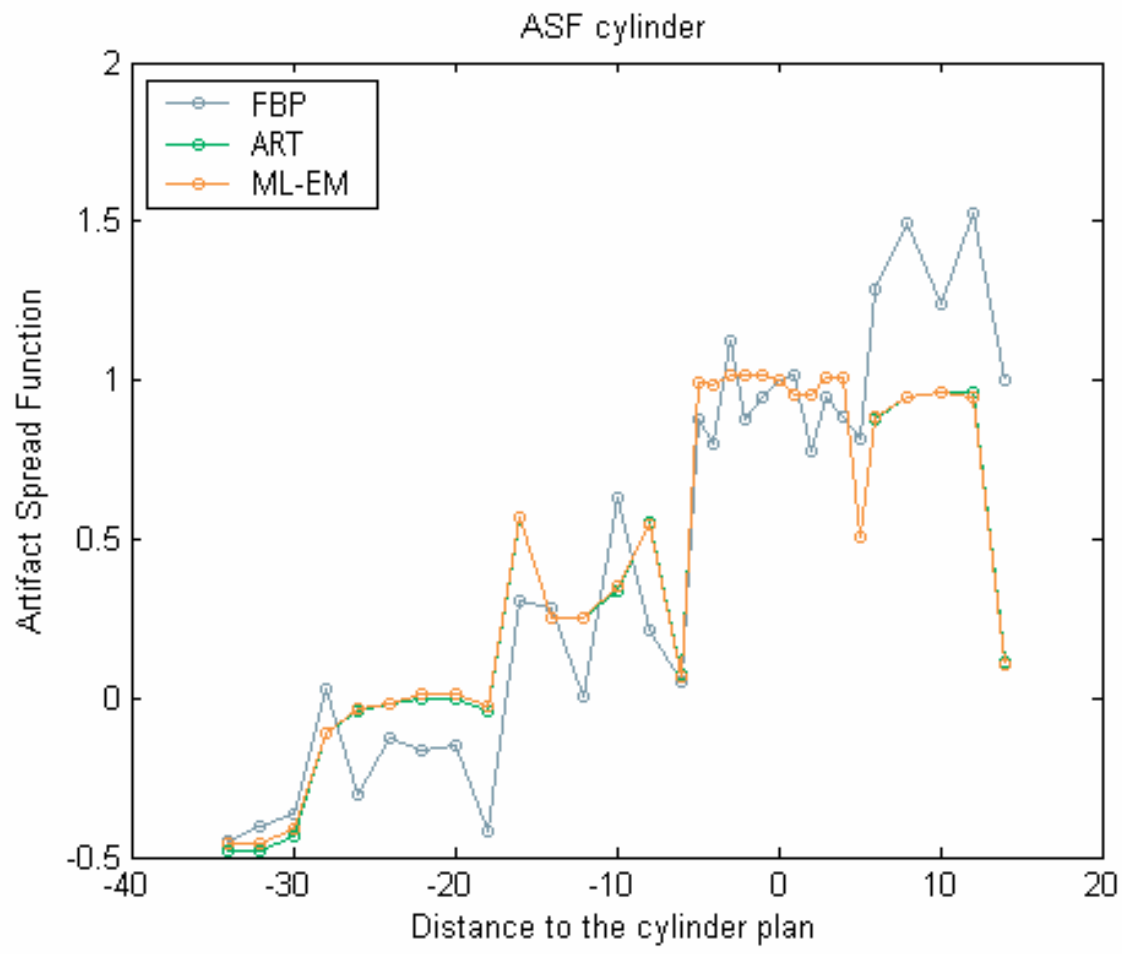

Figure 7.9 Artifact spread function of the cylinder object for the three algorithms. 
The time performance of an algorithm is an important indicator. Hence the runtime measurement is fundamental. Table 7.3 shows the duration, in seconds, of each reconstruction method.

Table 7.3 Runtime comparison between FBP, ART and ML-EM methods.

\begin{tabular}{|c|c|c|c|}
\hline \multicolumn{4}{|c|}{ Time performance } \\
\hline & $\begin{array}{c}\text { FBP } \\
\text { (no iteration) }\end{array}$ & $\begin{array}{c}\text { ART } \\
\text { (3 iterations) }\end{array}$ & $\begin{array}{c}\text { ML-EM } \\
\text { (3 iterations) }\end{array}$ \\
\hline seconds & 13.6400 & 4.8900 & 6.51500 \\
\hline
\end{tabular}

\subsection{Discussion}

The iterative methods, the ML-EM and the ART, took 3 iterations to get the final results. In both cases, more iterations did not produce image quality improvements. That was concluded by visual comparison between the results. The blur and the artifact spread did not change with the increase of the number of iterations.

One first superficial and visual comparison between the three algorithms, having the original volume on mind, leads us to think that the ML-EM and ART give better results than the FBP method, since the contrast of the last seems to be slightly worst.

The details can be discriminated from the background with the results from the three algorithms. The ellipsoid is the biggest detail defined in the phantom. Its existence can be seen in each plan of the volumes reconstructed on the three different manners. Its dimension suggests that it is larger than the simulated object. This is a known effect that results from the object distance to the detector, when the beam rays are not parallel between them. The further the detector is from the object, the greater is its spreading out, seeming larger than in the reality. Almost all the structures are well perceptible as theirs geometries and dimensions. The cylinders are well perceptible and theirs geometries are clear. The largest spheres (2 millimetres) are also visible on the reconstruction volumes. The smallest ones (1 millimetre) are not always well discriminated in the images, but they can also contribute to the variation in the grey level that is registered on the region where the spheres are localized.

With the FBP algorithm, in the initial slices the cylinder phantom has a slight deformation, seeming somewhat oval. This effect was expected from the beginning 
because it is characteristic of the tomosynthesis technique (Figure 5.3). It results from the utilization of a restricted angle to perform the projection acquisition. In opposition, the ML-EM and the ART algorithms do not present this artifact, which can denote that the performance of the iterative algorithms can be better in limited-angle cases, comparing to FBP.

The ML-EM and ART algorithms present the details contours well defined, comparing to the results from FBP. Note that the structures do not have the same horizontal spread as in the FBP algorithm. The x-ray beam is well defined, as the cylinder or the mass contours in the ML-EM and ART methods. Comparing the ART with the ML-EM, the results are similar.

The three algorithms present artifacts on theirs slices, by viewing unsharpness objects out of theirs plans. The artifacts results from the spread of the structures signal along the slices. The ellipsoid is large enough to cause its artifact spread along the whole slices on the three algorithms.

The FBP method generates more smoothed images than the other techniques. This smoothing arises from the filter application on the measured images in the beginning of the reconstruction process.

In all the methods the images present a grid artifact. This is a consequence of an error on the system matrix calculation. During the calculations, the contribution of the voxels in which the rays intersect them by the sides and not by the top, was not considered. This is not a consequence of the algorithm implementation or the images utilized. Figure 7.10 presents the result of a simple back projection of a uniform image using the system matrixes. Note that the grid is still present and is decreasing. The closer the source, the great the number of voxels in which the rays crossed them by theirs sides and not by theirs tops. 


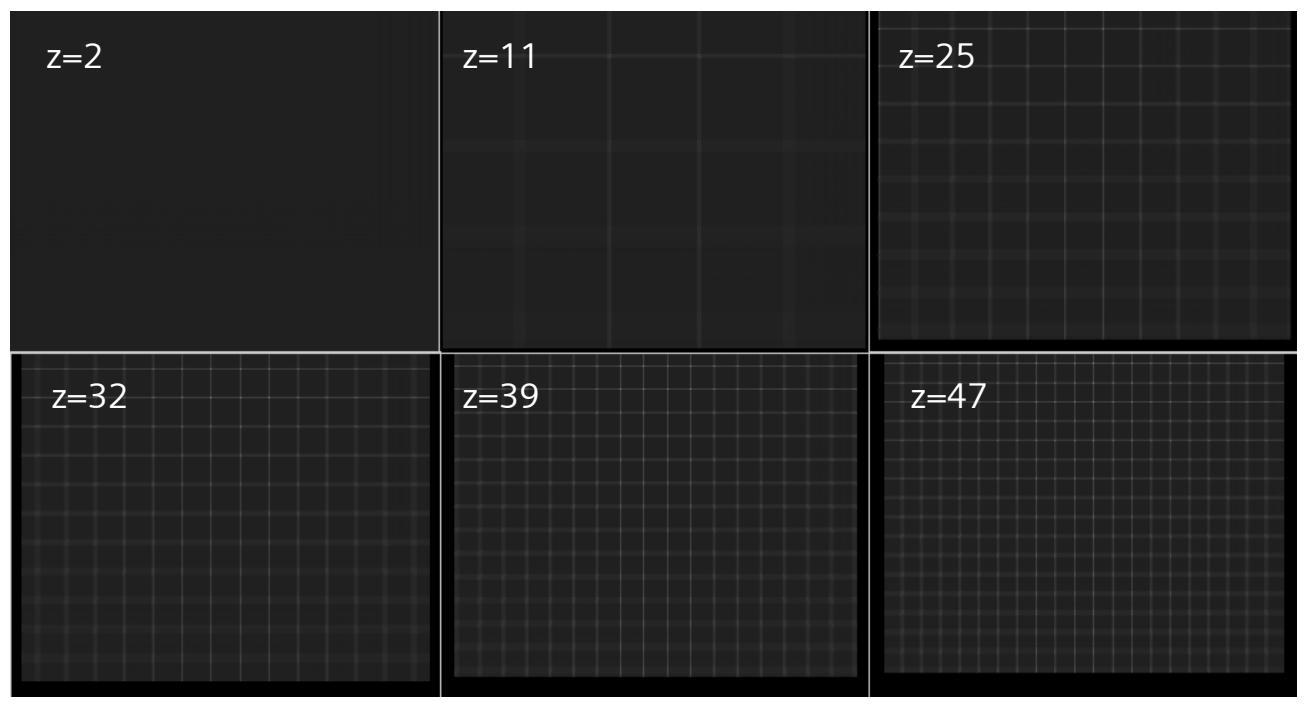

Figure 7.10 Slices 2, 11, 25, 32, 39 and 47 from a volume resultant from the back projection of a uniform image, using the system matrix constructed.

The resultant images from the FBP reconstruction present some artifact spreading along the object plan, the blur, more than in the ML-EM and ART algorithms. The blur disappears and the details limits become more delineate as the slices are closed to the object plan.

One of the indicators measured was the contrast-to-noise ratio. By the analysis of Table 7.1, the algorithms present a similar CNR for the larger detail, the ellipsoid. However the FBP method presents a worst CNR values for the sphere and the cylinder than the other algorithms. This confirms the visual analysis done above.

Comparing the ASF graphs, the similarity between the results from the ML-EM and the ART algorithms is notorious. These methods present an analogous behaviour on the artifact spread. On the ellipsoid case, ML-EM and ART show an increase of ASF before the object plan and a decrease after it. Nevertheless, the FBP presents a more accentuated increase of ASF before the object plan, evidencing better the plan where the ellipsoid is in fact. In relation to the sphere, the ASF is progressively decreasing along the volume slices, for the ART and the ML-EM algorithms. The graph shows that the spheres are well differentiated in the previous and nearest plans. The FBP presents an irregular behaviour, in which it seems that the spheres can be differentiated from the background along the whole volume, which is contradictory to the images. For the cylinders, the three algorithms present values of ASF that are increasing with the proximity to the cylinder plan. Only the FBP algorithm present increases beyond the cylinder plan, evidencing the cylinders better in slices above than in slices below. 
The sharp variation on the signal can interfere on the ASF measurements. The irregular behaviour of the artifact spread function is resultant from the grid presented on the images.

Making a comparison between the runtime for the three methods, the ML-EM and ART algorithms present similar runtimes and the FBP present the highest value. This was not expected, since the ML-EM and ART are iterative methods which should take more time of execution. However, the number of iterations performed is low: 3 in both cases; and the FBP algorithm has one calculation more, which is the filtration step. Anyway, these differences are not significant, as the number of iterations should increase when images of higher resolution were used. The runtime of an algorithm, in spite of being comparatively significant, it cannot be a reason to disregard it, if it does not delay the normal workflow of a service. 



\section{Conclusions}

The main purposes of this work were to acquire simulated data and used it to compare $3 \mathrm{D}$ reconstruction algorithms. The goal would be the development of a new algorithm.

The Monte Carlo simulation was the used method to create the simulated data. The images obtained from it were a fundamental tool. Without them, the reconstruction process could not be performed. The simulated images are the best form to perform an evaluation of a reconstruction technique, since the original volume is well known and it can be compared with the reconstructed volume. Different structures were introduced on a phantom to assess the algorithms behaviour when there are signal variations in the image.

It was not found any work of breast tomosynthesis performed on GATE, maybe because the tool for this purpose of simulation is a novel device. The work done on Monte Carlo simulations during this project was considered relevant to the scientific community since it resulted on a publication on the World Congress of Medical Physics and Biomedical Engineering.

The image reconstruction process was performed on the MATLAB software, and the selected algorithms for implementation have been widely accepted in the scientific 
community. Filtered back projection, algebraic reconstruction technique and the maximum-likelihood expectation-maximization were the implemented and tested methods on the present work and they are example of an analytical, iterative and statistical, respectively. The three algorithms were constructed from the base, having in mind the specially geometry of the DBT. The matrix system was the method chosen to model this system and to perform the reconstruction. Each projection has a correspondent matrix that was previously constructed and saved.

The three algorithms, which seem never to have been compared before for breast tomosynthesis, presented similar results. The details in the phantom can be distinguished from the background by the implementations of any one of the three algorithms. The results of the 3D image reconstruction allow the discrimination of smaller structures, the spheres which could not be differentiated on the simple projections that result from the simulations. This proves that the accuracy of Digital Breast Tomosynthesis can be better than the mammography.

The FBP algorithm presented more blurring images than the ML-EM and ART algorithms. However, it was the more capable one localizing the structures on the 3D space, including the smaller details.

Digital Breast Tomosynthesis has a strong potential to replace the mammography. It can be considered as an improvement to mammography that currently is the technique of reference for screening and diagnostic of breast diseases. The DBT will solve several failures of mammography and that can be proved by this work. The phantom details are better perceived in tomosynthesis than in mammography and it is possible to estimate the object localization on the space. However, the number of images that are analysed on tomosynthesis seems to be elevated. The visualization of the entire slices can be exhausting and it can result on a loss of attention by the radiologist.

\subsection{Work Limitations}

The first big step of the work was the reproduction of the tomosynthesis images on a platform for simulations. The Monte Carlo simulation demonstrated to be an arduous task. Firstly, an adaptation to the Linux environment was needed and the GATE installation was quite complex due to compilation errors. Thereafter, the familiarization process with the CTscanner, the new functionality available on GATE, was changeling since there is little information about it on GATE Users Guide, because it is a novel tool. 
After the familiarization process with the GATE program, the simulations could not be completed using the real size of the detector pixel, i.e., given the elevated requirements of memory needed to complete the simulations. The solution passed by the utilization of lower resolution images, nevertheless, being equally useful.

A significant part of these images were acquired in Milipeia, the supercomputer of the University of Coimbra, because the workstation available for this project had limited memory to enable the accomplishment of the simulation.

The time needful to complete the initial proposed work plan was underestimated. Not all the tasks could be completed in the proposed time and, some of them become unneeded due to changes on the plan. These did not contribute directly to the results but, indeed, it improved the acquired knowledge.

On the initial work plan, the reconstruction process should be performed on the STIR (Software for Tomographic Image Reconstruction) platform. In order to use it, the output from the GATE should be adapted to a sinogram. Moreover, the source code of STIR should be changed to accept that sinogram and to complete the reconstruction accordantly with the geometric characteristics of DBT. The source code of STIR is written on the programming language $\mathrm{C}++$. Hence, to perform the necessary alterations, the learning of the $\mathrm{C}++$ programming language was done on the beginning of this project. The STIR installation was also a time consuming task. The installation of a different Linux distribution seemed to solve the problem and allowed the complete STIR installation. Despite all the efforts done to allow the STIR utilization, the work plan was changed. The sinograms construction and the code alterations seemed to be more difficult to perform than the construction of an entire code already adapted to the DBT geometry. Despite the spent time, it was acquired the knowledge about the reconstruction platform, STIR, and about the $\mathrm{C}++$ language.

\subsection{Future Work}

The grid artifact on the system matrixes construction was detected only during the reconstruction phase. The time available was not enough to allow the matrixes correction. However, this work is already in progress.

Images with superior resolution are needed to better assess the image reconstruction methods. The simulated images should be redone with a detector resolution more 
approximated to the real detectors used on the real mammographic acquisitions, to better simulate the tomosynthesis exam.

As a recent technique, the Digital Breast Tomosynthesis has a wide area of intervention with possible improvements. On the continuity of this work, the most important areas to focalize are the issues related with the image processing. The existent algorithms need greater developments and require optimizations. The algorithms have to be adapted to respond on a better manner to the limitations of the tomosynthesis technique. Since it has a limited angle to acquire the projections (50 degrees) and the number of projections is very low (generally are taken 11 projections, but the number can increase to 17 projections). More existent methods should be implemented and tested. Accordantly with the results obtained for the algorithms performance, some of them should be optimized and the development of a new algorithm can occur. This is the main goal for the continuity of the present work.

The angle between two successive image acquisitions used in this work was 5 degrees. We think that this value can influence the performance of an algorithm. Hence, on a future work, this aspect should be tested to analyse the alteration on the new algorithm performance.

The thickness of the reconstructed volume slices was chosen because it was the most encountered value on the literature and it was, apparently, the most adequate thickness to use on that phantom definition. However, it is not known the value for the greater thickness to use on the breast tomosynthesis. The influence of this aspect on the quality of the reconstructed images should be analysed in order to determine the optimal thickness.

The use of simulated images using analytical phantoms is the better way to perform the assessments of the reconstruction algorithms. As it was explained, these phantoms allow the acquisition of regular and controlled data, and allow the isolation of the errors that come from the reconstruction algorithm, without the interference of physical measurement errors. Besides that, the main point on the simulated data usage is the fact that the used model is exactly known, allowing the better comparison between the reconstructed images and the original object. However, on a following step, the algorithms must be tested with real data. The algorithm performance must be tested with real data using clinical images. 


\subsection{Final Work Assessment}

This project allowed the increase of the knowledge about Monte Carlo simulations and image reconstruction and also about diverse tools available to perform them. But apart the whole specific work done in these areas, much more was acquired. The inclusion of this work on a company was a real asset. It represents a solid progress on my skills: in the sense of responsibility and autonomy increased; the network enlargement; and several opportunities to the growth of my professional competences. During the last months, it was given the opportunity to: visit an Imaging Service where the mammographic exam is performed; do some online formations that the company provides to its collaborators; participate on the Medical Physics Workshop realized in Aveiro; to assist the symposium entitled "Monte Carlo Simulations: Applications to Mammography" given by a reputable name on the mammographic simulations, Matthaios Koutalonis; and participate on the World Congress of Medical Physics and Biomedical Engineering.

This project was integrated on a group of research, in which several PhD and other MSC students are included. They are students from different areas with different formations, becoming complementary in terms of areas of knowledge. These researchers have important scientific projects in hand. They are people with enormous knowledge, with a great sense of mutual help. It was an enormous privilege to integrate this group and I am really grateful for all that they did for this work and for me.

This work was ambitious and very exigent, but inspiring and challenging. 


\section{Bibliography}

1. World Health Organization Webpage. [cited 9-July-2009]; Available from: http://www.who.int/mediacentre/factsheets/fs297/en/index.html.

2. Society, A.C., Cancer facts and figures 2008. 2008.

3. Saúde, D.G.d. Plano nacional de saúde - cancro. 2004 [cited 9-July-2009]; Available from: http://www.dgsaude.min-saude.pt/pns/vol2_224.html.

4. Laço. Cancro da mama em Portugal. [cited 14-July-2009]; Available from: http://www.laco.pt.

5. Saúde, P.d. Guia de Apoio à Mulher com Cancro de Mama 2005 [cited 14-July2009]; Available from: www.portaldasaude.pt.

6. Porto, I.P.d.O.d. Registo oncológico 2007. 2008 [cited 9-July-2009]; Available from: $\quad$ www.ipoporto.min-saude.pt/NR/rdonlyres/85BB8B9A-81F2-43AF-810084D354C5116E/15267/IPO_2007.pdf.

7. Society, A.C. Global Cancer Facts \& Figures 2007. 2007 [cited 9-July-2009]; Available from: http://www.cancer.org/docroot/STT/content/STT_1x_Global_Cancer_Facts_and_Fig ures_2007.asp.

8. American Cancer Society Webpage. [cited 10-July-2009]; Available from: http://www.cancer.org/docroot/CRI/content/CRI_2_4_3X_How_is_breast_cancer_st aged_5.asp.

9. Otto, S.J., et al., Initiation of population-based mammography screening in Dutch municipalities and effect on breast-cancer mortality: a systematic review. The Lancet, 2003. 361: p. 1411-1417.

10. Reddy, M. and R. given-Wilson, Screening for breast cancer. Women's health medicine, 2006. 3(1).

11. Parvinen, I., et al., Service screening mammography reduces breast cancer mortality among elderly women in Turku. Journal of Medical Screening, 2006. 13(1): p. 34-40.

12. Tabár, L., et al., Beyond Randomized Controlled Trials Organized Mammographic Screening Substantially Reduces Breast Carcinoma Mortality. American Cancer Society, 2006: p. 1724-1731.

13. Manuela Gonçalo, et al., Imagiologia Clínica Princípios e Técnicas, Coimbra: Francisco de Mascarenhas.

14. Saúde, A.C.d., Plano Nacional de Prevenção e Controlo das Doenças Oncológicas 2007/2010. 2007.

15. Poplack, S.P., et al., Mammography in 53,803 Women from the New Hampshire Mammography Network1, in Radiology 2000. p. 832-840.

16. Taplin, S., et al., Mammography Facility Characteristics Associated With Interpretive Accuracy of Screening Mammography. JNCI, 2008. 100(12): p. 876.

17. Sidharth, et al., Mammographic Diagnosis of Breast Carcinoma: An Institutional Experience. JNMA, 2008. 47(170): p. 62-65. 
18. Kavanagh, A.M., et al., The sensitivity, specificity, and positive predictive value of screening mammography and symptomatic status. J Med Screen, 2000. 7: p. 105110.

19. Siemens S.A., S.H., Company Presentation, Portugal. 2009.

20. Laterjet, M. and a.R. Liard, Anatomia Humana. Vol. 2. 1999, Madrid: Editorial Médica Panamericana.

21. Breast anatomy and physiology. 2008 18-Mars-2008 [cited 30-October-2008]; Available from: www.imaginis.com/breasthealth/breast_anatomy_print.asp.

22. Cancer Line Webpage. [cited 17-November-2008]; Available from: http://www.cancerline.com/gUserFiles/breastDev_fig_3.gif.

23. Kasper, D., et al., Medicina Interna. Vol. I. 2006, Rio de Janeiro: Mc Graw Hill.

24. Cancro, L.P.C.o. Cancro da mama. [cited 10-July-2009]; Available from: www.ligacontracancro.pt.

25. Oldnall, N. Xray2000 Website. [cited 14-November-2008]; Available from: http://www.e-radiography.net/radpath/d/dcislatest.gif.

26. Stephan, P. What You Need to Know About Breast Cancer Symptoms. 2004 4Mars-2007 [cited 3-November-2008].

27. ADAM. Breast Cancer Health Information Webpage. [cited 17-November-2008]; Available from: http://health.nytimes.com/health/guides/disease/breastcancer/symptoms.html.

28. Management of Breast Diseases Website. [cited 8-May_2009]; Available from: http://www.breastdiseases.com/stage.htm.

29. Breast cancer survival rate. [cited 8-June-2009]; Available from: http://www.fireworkszone.com/infoonfinance/cancer/breast_cancer_survival_rate. html.

30. Breast exam. 2008 [cited 4-November-2008]; Available from: http://www.mayoclinic.com/health/breast-self-exam/WO00026.

31. Nelson, M., M. Lechner, and D. Whaley, ICSI Technology Assessment Report, in Institute for Clinical Systems Improvement. 2004.

32. America, R.s.o.N. RadiologyInfo. 2009 21-June-2009 [cited 9-July-2009].

33. Stephan, P. Diagnosis of Breast Cancer. 2009 29-January-2009 [cited 9-July2009]; Available from: http://breastcancer.about.com/od/diagnosis/a/diagnosis_ov.htm.

34. Pisano, E.D., et al., Diagnostic Performance of Digital versus Film Mammography for Breast-Cancer Screening. The New England Journal of Medicine, 2005. 17(353): p. 1773-1783.

35. Improving Methods for Breast Cancer Detection and Diagnosis. [cited 9-July2009]; Available from: http://www.cancer.gov/cancertopics/factsheet/Detection/breast-cancer.

36. e-images Webpage. [cited 10-November-2008]; Available from: https://www.eimages.medical.siemens.com/eimages.

37. Rangayyan, R.M., Biomedical Image Analysis. Biomedical Engineering Series, ed. M.R. Neuman. 2005, University of Calgary, Alberta, Canada: CRC press. 
38. Wang, S.-C. and R.L. Birdwell. Magnetic Resonance Mammography. 2007 27January-2007 [cited 6-November-2009].

39. Diekmann, F. and U. Bick, Tomosynthesis and contrast-enhanced digital mammography: recent advances in digital mammography. European Radiology, 2007. 17(12): p. 3086-3092.

40. Schillaci, O. and J.R. Buscombe, Breast scintigraphy today: indications and limitations. european Journal of Nuclear Medicine and Molecular Imaging, 2004. 31(1): p. S35-S45.

41. Medicine, S.o.N., Procedure guideline for breast scintigraphy. Society of Nuclear Medicine, 1999.

42. Torres, S., et al. (2003) Scintimammography in the evaluation of axillary lymph node invasion in patients with breast cancer. Alasbimn Journal Volume,

43. Breast Thermography Webpage. [cited 11-November-2008]; Available from: http://www.breastthermography.com/images/NML-2-m.jpg.

44. Cedars-Sinai. 2008 [cited 1-Setember-2009]; Available from: www.csmc.edu/6973.html.

45. Almeida, P. Issues in PEM Image Reconstruction. in Workshop on Positron Emission Mammography 2002. Lisbon.

46. Smith, A.P., P.A. Hall, and D.M. Marcello, Emerging Technologies in Breast Cancer Detection. 2006.

47. Systems, M.C., Digital Breast Tomosynthesis (DBT) A New Tool for Detecting Breast Cancer. 2005.

48. Lima, J.J.P.d., Técnicas de diagnóstico com raios $X$ Aspectos Físicos e Biofísicos. 2005, Coimbra: Imprensa da Universidade de Coimbra.

49. Colourtherapy Web page. [cited 20-November-2008]; Available from: http://www.colourtherapyhealing.com/colour/images/electromagneticspectrum.jpg.

50. Bronzino, J.D., The Biomedical Engineering Handbook. Second edition ed, ed. R.C. Dorf. Vol. I. 2000, University of California: CRC press, IEEE press.

51. Merriam-Webster. Encyclopœdia Britannica - the Online Encyclopedia. 2006 [cited 20-November-2008]; Available from: http://cache.eb.com/eb/image?id=72253\&rendTypeld=35.

52. Environment, Health \& Safety. 8-September-2005 [cited 20-November-2008]; Available from: http://ehs.unc.edu/training/self_study/xray/fig3.gif.

53. LabSpace Webpage. [cited 12-February-2009]; Available from: http://labspace.open.ac.uk/file.php/3393/formats/S809_1_1.0_rss.xml.

54. Understanding $X$ Ray and Its History. [cited 12-February-2009]; Available from: http://asxrt.org/index_files/Page588.htm.

55. Trestrail, L., Radiation and Interaction of Radiation with Matter. 2008.

56. Oldnall, N. Xray2000 Website. 4-May-2009 [cited 8-June-2009]; Available from: http://www.e-radiography.net/radtech/u/unsharpness1.jpg.

57. Leroy, C., Principals of Radiation Interaction in Matter and Detection. 2004, Singapure: World Scientific Publishing. 
58. Trestrail, L. Radiation and Interaction of Radiation with Matter. 13-October-2008 [cited 20-November-2008]; Available from: www.cs.unm.edu/ compmed/seminars/LT_MedPhys2.pdf.

59. Thomenius, K. and B. Roysam. Introduction to Subsurface Sensing and Imaging Systems. $\quad$ [cited 21-July-2009]; Available from: http://www.ecse.rpi.edu/censsis/SSI-Course/Lectures.2005/Lecture\%2006\%20\%202005/SSI-Lecture06-2005.ppt.

60. Transmitted Intensity and Linear Attenuation Coefficient [cited 20-February2009]; Available from: Transmitted Intensity and Linear Attenuation Coefficient

61. Epstein, C.L., The Mathematics of Medical Imaging. 2001: Pennsylvania.

62. Frangi, A., Introduction to Biomedical Imaging.

63. Johns, P.C. and M.J. Yaffe, X-ray characterisation of normal and neoplastic breast tissues. Phys. Med. Biol., 1987. 32(6): p. 675-695.

64. Malur, S., et al., Comparison of written reports of mammography, sonography and magnetic resonance mammography for preoperative evaluation of breast lesions, with special emphasis on magnetic resonance mammography. Breast Cancer Research, 2001. 3(1): p. 55-60.

65. Mammogram. [cited 17-November-2008]; Available from: http://www.medic8.com/healthguide/articles/mammogram.html.

66. Young, K.C. and D.Kitou, Review of Literature on Digital Mammography in Screening. 2005, NHS Cancer Screening Programmes. p. 16.

67. Planmed Advanced Breast Care Solutions. Volume,

68. Janke, W., Statistical Analysis of Simulations: Data Correlations and Error Estimation. Quantum Simulations of Complex Many-Body Systems: From Theory to Algorithms, 2002. 10: p. 423-445.

69. Jan, S., et al., GATE Users Guide. 2008, Marseille. 206.

70. Shorey, J., Stochastic Simulations for the Detection of Objects in Three Dimensional Volumes: Applications in Medical Imaging and Ocean Acoustics, in Department of Electrical and Computer Engineering 2007, Duke University: Duke. p. 257.

71. Zaidi, H. and M.R. Ay, Current status and new horizons in Monte Carlo simulation of X-ray CT scanners. Med Bio Eng Comput, 2007. 45: p. 809-817.

72. Kirk, B.L., G. Sjoden, and A. Haghighat. Activities of the computational medical physics working group. in International Conference on the Physics of Reactors "Nuclear Power: A Sustainable Resource". 2008. Casino-Kursaal Conference Center, Interlaken, Switzerland,.

73. Geant4 webpage. [cited 26-February-2009]; Available from: http://www.geant4.org/geant4/

74. MCNP A General Monte Carlo N-Particle Transport Code Webpage. 29-January2004 [cited 26-February-2009]; Available from: http://mcnpgreen.lanl.gov/index.html.

75. Fluka. Fluka [cited 26-February-2009; Available from: http://www.fluka.org/. 
76. Niita, K., Nuclear Reaction Models in Particle and Heavy lon Transport code System PHITS, in 1st Workshop on Accelerator Radiation Induced Activation. 2008: Paul Scherrer Institut, Switzerland.

77. Bonifácio, D., H. Murata, and M. Moralles, Monte Carlo Simulation of X-ray spectra in diagnostic radiology and mammography using Geant4, in 2005 International Nuclear Atlantic Conference - INAC 2005. 2005.

78. Ay, M., et al., Monte Carlo Simulation of X-ray spectra in diagnostic radiology and mammography using MCNP4C. Phys. Med. Biol. , 2005. 49: p. 4897-4917.

79. Ng, K.P., C.S. Kwok, and F.H. Tang, Monte Carlo simulation of x-ray spectra in mammography. Phys. Med. Biol., 2000. 45: p. 1309-1318.

80. Spyrou, G., et al., A Monte Carlo simulation model of mammographic imaging with x-ray sources of finite dimensions. Phys. Med. Biol., 2002. 47: p. 917-933.

81. Boone, J.M., et al., Development and Monte Carlo Analysis of Antiscatter Grids for Mammography. Technology in Cancer Research \& Treatment, 2002. 1(6): p. 441447.

82. Mowlavi, A.A., X-ray spectra calculation for different target-filter of mammograms using MCNP Code. Iran. J. Radiat. Res, 2005. 3(3): p. 129-133.

83. Delis, H., et al., The influence of mammographic $X$-ray spectra on absorbed energy distribution in breast: Monte Carlo simulation studies. Radiation Measurements, 2005. 39(2): p. 149-155.

84. Delis, H., et al., DOSIS: a Monte Carlo simulation program for dose related studies in mammography. European Journal of Radiology, 2004. 54: p. 371-376.

85. Dance, D.R., et al., Influence of anode/filter material and tube potential on contrast, signal-to-noise ratio and average absorbed dose in mammography: a Monte Carlo study. The British Journal of Radiology, 2000. 73: p. 1056-1067.

86. Sechopoulos, I. and C.J. D'Orsi, Glandular radiation dose in tomosynthesis of the breast using tungsten targets. Journal Of Applied Clinical Medical Physics, 2008. 9(4): p. 161-171.

87. Morera, A.F., et al., Breast Tumors: Composition of Microcalcifications. Radiology, 1988. 169: p. 325-327.

88. ALECRIN, I.N., et al., Calcificações mamárias: quando biopsiar? Revista da Associação Médica Brasileira, 2001. 47(1): p. 10-11.

89. Weddellite - Mineral Date Publishing. 2001.

90. Moritz, J.D., et al., Microcalcifications in Breast Core Biopsy Specimens: Disapperance at Radiography after Storage in Formaldehyde. Radiology, 1996. 200: p. 361-363.

91. Segars, W.P., et al., Extension of the 4D NCAT Phantom to Dynamic X-ray CT Simulation. Nuclear Science Symposium Conference Record, 2003 IEEE, 2003. 5: p. 3195- 3199.

92. III, J.T.D. and D.J. Godfrey, Digital x-ray tomosynthesis: current state of the art and clinical potential. PHYSICS IN MEDICINE AND BIOLOGY, 2003. 48(R65-R106).

93. Suryanarayanan, S., et al., Comparison of Tomosynthesis Methods Used with Digital Mammography. Acad Radiol, 2000. 7: p. 1085-1097. 
94. Suryanarayanan, S., et al., Evaluation of Linear and Nonlinear Tomosynthetic Reconstruction Methods in Digital Mammography. Acad Radiol, 2001. 8: p. 219224.

95. Wu, T., et al., Tomographic mammography using a limited number of low-dose conebeam projection images. Med. Phys., 2003. 30(3): p. 365-380.

96. Wu, T., et al., Digital tomosynthesis mammography using a parallel maximum likelihood reconstruction method. SPIE, 2004. 5368(1): p. 1-11.

97. Sompel, D.V.d. and M. Brady, Simultaneous Reconstruction and Segmentation Algorithm for Digital Breast Tomosynthesis, in Biomedical Imaging: From Nano to Macro, 2008. ISBI 2008. 5th IEEE International Symposium. 2008. p. 1035-1038.

98. Bleuet, P., R.e. Guillemaud, and I.E. Magnin, Resolution Improvement in Linear Tomosynthesis with an Adapted 3D Regularization Scheme. Proceedings of SPIE, 2002. 4682: p. 117-125.

99. Chen, Y., J.Y. Lo, and J.T.D. III, Impulse response analysis for several digital tomosynthesis mammography reconstruction algorithms. Proceedings of SPIE, 2005. 5745: p. 541-549.

100. Chen, Y., et al., Gaussian frequency blending algorithm with Matrix Inversion Tomosynthesis (MITS) and Filtered Back Projection (FBP) for better digital breast tomosynthesis reconstruction. Proceedings of SPIE, 2006. 6142.

101. Zhou, L., et al., Low-Contrast Lesion Detection in Tomosynthetic Breast Imaging Using A Realistic Breast Phantom. Proceedings-Spie The International Society For Optical Engineering5368, 2006. 6142(3): p. 61425A.

102. Zhang, Y., et al., A comparative study of limited-angle cone-beam reconstruction methods for breast tomosynthesis. Med. Phys., 2006. 33(10): p. 3781-3795.

103. Sidky, E.Y., et al. Practical iterative image reconstruction in digital breast tomosynthesis by non-convex TpV optimization. in Medical Imaging 2008: Physics of Medical Imaging 2008. San Diego, CA, USA

104. Matela, N.M.d.P.L., 2D Iterative Image Reconstruction for a Dual Planar Detector for Positron Emission Mammography, in Departamento de Física. 2008, Universidade de Lisboa: Lisboa.

105. Thompson, M. Contrast to Noise. 2003 21-November-2003 [cited 20-July-2009]; Available from: http://www.phys.cwru.edu/courses/p431/notes2003/node123.html. 


\section{Annex 1}

GATE Mammogram Simulation of NCAT Breast Phantom

Isabel Catarina Duarte ${ }^{1,2,}$, Liliana Caldeira ${ }^{2,3}$, Filipe Soares ${ }^{2,4}$, José Silvestre Silva ${ }^{1,5}$, Filipe Janela ${ }^{2}$.

'Physics Department, Faculty of Sciences and Technology of University of Coimbra Coimbra, Portugal

${ }^{2}$ Siennens S.A. Healthcare Sector, Porto, Portugal

${ }^{3}$ Instituto de Biofisicae Engenharia Biomédica, Lisboa, Portugal

${ }^{4}$ Universidade da Beira Interior, Covilhă, Portugal

${ }^{5}$ Instrumentation Centre, Faculty of Sciences and Technology of University of Coimbra

*Comesponding author e-mail: isabel.duarte @siemens.com

Absirad - In this paper, we aim to acquire simulated mammograms. This simulated data is performed on GATE (Geant4 Application for Tomographic Emission), a platform for simulation based on Monte Carlo methods. On this software, a particular tool, the new CTscanner, is used to software, a particular tool, the new CTscanner, is used to
simulate the interation between $\mathrm{X}$-ray and tissues. We hope also to demonstrate how the new CTscanner system can be used. The NCAT phantom is used to obtain data, where only the volume of the breast is employed to achieve the simulation. Geometric phantoms are also used for simulation of more simple objects. The parameters for the reproduction of manum manmograms are introduced accordingly the Siemens Mammomat lnspiration man ography unit, since this is a recent device. The aim of this work is to get simulated mammograms from different angles of the same object, which will allow the access of tri-dimensional reconstruction for Digital Breast Tomosynthesis. We present and discuss the results obtained using the proposed simulations.

Keywords- CTscanner, Digital Breast Tomosynthesis, GATE, Mammography, MonteCarlo me thods.

\section{INTRODUCTION}

Breast cancer, one the most pressing health issues of our time, is generally asymptomatic at the early stages of the disease. This makes the screening and early diagnostic disease. This makes the screening and early diagnostic
essential to improve the chances of a successful treatment. Mammography is the gold standard for breast cancer screening and diagnostic, since it has high sensitivity and high specificity at low cost. Nevertheless the fact of 3D anatomical information being projected into a $2 \mathrm{D}$ image plane causes some problems as overlapping tissue, loss of sensibility and specificity between tissues. This can mask a tumor or show a false-positive that will result on a recall. The possibility of 3D reconstruction of mammograms will solve these problems: increases sensibility and specificity, allowing distinguish malignant from benign tissue; allows the 3D visualization without overlapping tissues: admits recalls and its costs, and assesses the shape, size and location of a lesion [1-4].

GATE Marnmogram Simulation of NCAT breast phantom doc
In this work, in order to compare 3D reconstruction algorithms, simulated data and real mammograms will be used. Monte Carlo simulation will be an important tool to assess the image reconstruction algorithms, since it is possible to know the ground truth [5].

The GATE (Geant4 Application for Tomographic Emission) software was created for simulation of interactions between elementary particles and matter on Medical Physics area, using Monte Carlo methods. GATE, and specifically the CTscanner tool included therein are an important device to obtain simulated mammograms, given the stochastic nature of the process such as the interaction between the $\mathrm{X}$-rays with tissues, the $\mathrm{X}$-ray generation and detection [5]. The CTscanner is a system recently included in GATE and therefore, its use is not well known.

Siemens Mammomat Inspiration is a digital mammography unit, in which X-rays are used to produce images from breast anatomy [6]. It was the chosen device to reproduce on the simulation system.

4D NURBS-based Cardiac-Torso (NCAT) is a phantom that provides a realistic model of anatomy and physiology of human body. NCAT phantom is widely used on nuclear medicine imaging research, but it can also be applied on $x$ ray diagnostic techniques, since the phantom is used to define not only maps of biological activity distribution, but also maps of attenuation coefficient distribution [7].

Only a 3D region of this phantom is used: the volume corresponding to breast.

There are some works based on Monte Carlo methods to simulate mammographic exams, but with distinct intents. To understand the diagnostic $x$-ray energy spectra, simulations were performed on Geant 4 using different combinations of targets, filters and tube voltages [8]. Similar studies were completed using ITS code [9] and MCNP4C code [10], also based on Monte Carlo methods. 
II. MAterial AND MEthods

\section{A. NCAT phantom}

To perform the simulation of mammograms, a realistic phantom of breast is used: the NCAT phantom. On the aim of this work, only the breast volume is needed. Thus, the NCAT phantom was utilized as a phantom of a normal woman. It was cropped and just the attenuation coefficient distribution map referent to the breast volume is used. The resulting matrix is a $44 \times 43 \times 49$ voxels matrix. Each voxel is a $2 \times 2 \times 2$ millimeters cube.

Transversal, coronal and sagital slices of this phantom breast are illustrated on Fig. 1, Fig. 2 and Fig, 3, respectively. These images are representations of the map of attenuation coefficient distribution obtained from NCAT program.

The phantom used on this simulation corresponds to a large breast with $8 \times 8 \times 9$ centimeters, approximately.

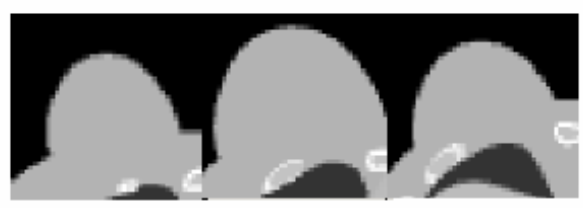

Fig. 1 Transversal slices of the breast phantom (from superior to inferior).

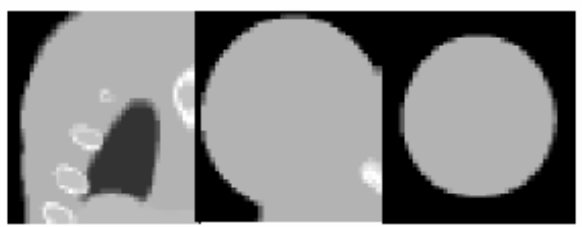

Fig. 2 Coronal stices of the breast phantom (from anterior to posterior).

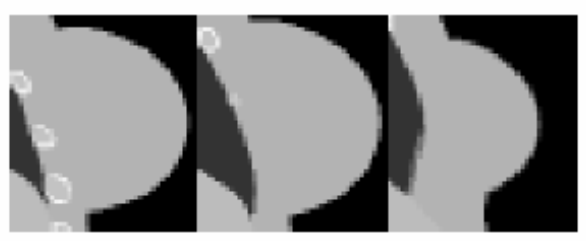

Fig, 3 Sagital slices of the breast phantom (from right to left).
The breast phantom is similar to a breast on a prone woman body and not as compressed breast. Since the simulated data is used on tri-dimensional reconstruction of Digital Breast Tomosynthesis (where the breast will suffer just a small compression to maintain it motionless), this is not a problem.

\section{B. Hardware}

Siemens Mammomat Inspiration is the mammography unit chosen, which characteristics are used to construct all system for the simulation. It was the mammography unit chosen as it is a recent device.

The parameters introduced are accordant to the breast size. Technical specifications, such as the voltage and the amperage, are regulated accordingly the thickness of the breast. This regulation can be done by the radiologist or by the automatic OPDOSE function. With this option, the exposure parameters as voltage and amperage are programmed based on breast thickness. We use the operated values to a thickness breast of 9 centimeters.

\section{GATE}

GATE is a platform for simulation, based on Geant 4 . The recent CTscanner system allows the simulation of the interaction radiation-matter on tomography using $\mathrm{X}$-rays.

On GATE, the materials used to construct the system and theirs characteristics can be found on a database. This has a range of different materials, including breast tissue.

In this system, the parameters are introduced as realistic as possible. The average photon energy and the number of photons per second are estimated from calculations using the voltage and amperage given by mammography unit. Other parameter, the distance between the source and the detector, can be modified from exam to exam. We will use the usual value. The correspondence between technical specifications of Mammomat Inspiration (usual for a 9 centimeters thickness breast) and the parameters that is introduced on GATE CTscanner can be seen on Table 1. The geometry created on GATE and the X-ray unit of Mammomat Inspiration are illustrated on Figure 4 


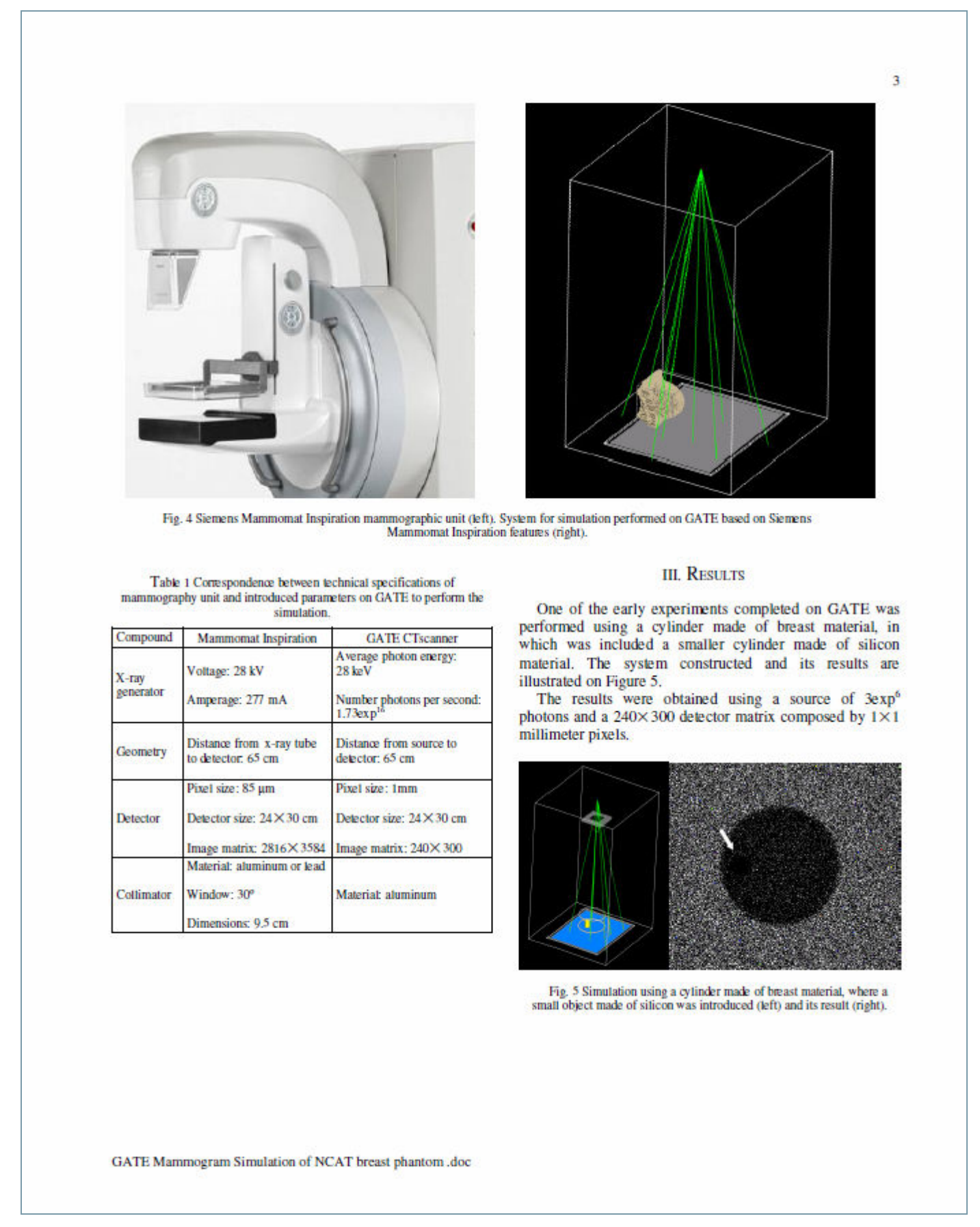


A new system is constructed using the breast phantom from NCAT.

The Figure 6 shows the number of photons that reaches the detector matrix (black is the null counting and white represents a detected photon) with the phantom.

The breast phantom is perfectly seen on the simulated data, although the low photons intensity and the large pixel size. The influence of the breast thickness variation is visible by the photons counting, as expected. It is clearly visible in the image, that the base of the breast has less photon count than the top of the breast. This is related to the number of photons that are attenuated by breast tissue. The attenuation depends on the thickness of tissue, as we can see by the Beer-Lambert law (equation 1):

$$
I=I_{0} e^{-\mu x}
$$

Where $I_{o}$ and $l$ are the incident intensity and the intensity after cross the material, $\mu$ is the linear attenuation coefficient and $x$ the material thickness.

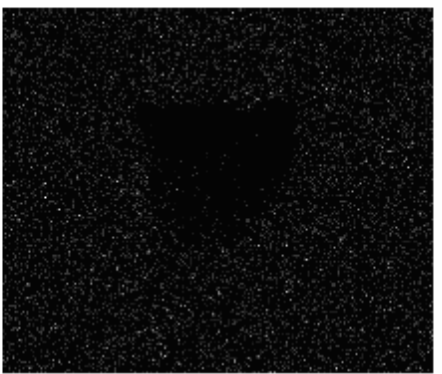

Fig. 6 Simulated mammogram acquired with phantom breast from NCAT.

\section{CONCUUSIONS AND FUTURE WORK}

These results obtained until now come from initial experiments. A very low photons intensity and a too large pixels size were used while other parameters were improved for sake of simplicity.

The results can be improved, decreasing the pixels size. The source must be reconstructed, considering it, not as a point, but as an area. It inclination must be also pondered, as well as the energy distribution of the photons. Other compound can be considerer, the compression plate, although its interference is minimal.

Knowing the results obtained until now, if these alterations were performed, we think that GATE may be a

GATE Mammogram Simulation of NCAT breast phantom doc good tool to perform the simulation and acquire the mammograms. Mammomat Inspiration can be modeled as an acquisition system in this software.

Hereafter, the acquisition will be performed from different angles maintaining the breast position in order to allow the tri-dimensional reconstruction. Some modifications will be introduced on the breast with the mean to reproduce breast diseases, such as tumor masses, cysts and micro-calcifications. Phantoms with breast implants will also be simulated. The objective is to assess the tri-dimensional reconstruction, knowing the breast phantom composition.

\section{ReFERENCES}

1. Jecng Mi Park,Edmund A. Franken, Megha Gare, Laurie L. Fajardo Loren T. Niklason (2007) Breast Tomosynthesis Present Loren T. Niklason (2007) Breast Tomosynthesis Present
Considerations and Future Applications, RadioGraphics 2007; 27:\$231-\$240

2 Ann-Katherine Carton, Jinging Li, Michael Albert, Sara Chen, Andrew D.A. Maidment (2006) Quantification for contrast-enhanoed digital breast tomosynthesis, Medical Imaging 2006: Physics of Medical Imaging

3. Lili Zhou, Jorge Oldan, Paul Fisher and Gene Gindi (2006) LowContrast Lesion Detection in Tomosynthetic Breast Imaging Using A Realistic Breast Phantom, Medical Imaging 2006: Physics of Medica lmaging

4. Diego Rivera, Dana Schan, Micha Moffie, David Kaeli (2007) Exploring Novel Parallelization Technologies for 3-D Imagin Applications 19th International Symposium on Computer Architecture and High Performance Computing

5. Jan S., Santin C. Strul D. et al (2008) GATE Users Guide.

6. Mammomat Inspiration, data sheet. (2008).

7. Segars W.P. Tsui B.M.W. Frey EC., Fishman E.K. (2003) Extension of the 4D NCAT phantom to dynamic X-ray CT simulation. Nuclear Science Symposium Conference Record, 2003 IEEE 5: pp 3195 - 3199 .

8. Bonifácio, D., Murata H., Moralles M. (2005) Monte Carıo Simulation of X-ray spectra in diagnostic radiology and mammography using Ceant4. 2005 International Nuclear Atlantic Conference - INAC 2005

9. Ng K, Kwok C, Tang F. (2000) Monte Carlo simulation of X-ray spectra in mammography. Phys. Med. Biol. 45: pp 1309-1318.

10. Ay M., Shahriari M., Sarkar S., Adib M., Zaidi H. (2004) Monte Carlo Simulation of $\mathrm{X}$-ray spectra in diagnostic radiology an mammography using MCNP4C. Phys. Med. Biol. 49: pp 4897-4917.

\section{Corresponding author:}

Author. Isabel Catarina Duarte

Institute: Universidade de Coimbra

Street: Rua Larga, 3004-5

City: Coimbra

Emait isabel.duarte siemens.com 


\section{Annex 2}

\section{D Medical Image Reconstruction}

Isabel Duarte ${ }^{1,2, *}$, Liliana Caldeira², Filipe Soares ${ }^{2}$, Filipe Janela², José Silvestre Silva ${ }^{1,3}$ 1 Physics Department, Faculty of Science
Rua Larga, $3004-516$ Coimbra, Portugal

${ }^{2}$ Siemens S.A. Healthcare Sector, Estrada Nacional 107, no. 3570 Freixieiro

-901 Perafita, Portugal

Corresponding author e-mail: isabel.duarte@siemens.com

Answers for life.

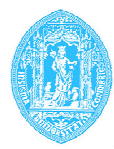

SIEMENS

\section{Aim}

To use different algorithms of 3D medical image reconstruction for implementation in mammography and later comparison between them. Simulated data and real mammograms will be used to implement the algorithms.

3D Medical Image Reconstruction is a project of the Integrated Master in Biomedical Engineering of University of Coimbra.

\section{Project scope}

Breast cancer is one of the cancer types with higher incidence. This breast disease is generally asymptomatic at the early stages of the disease. This makes the screening and early diagnostic essential to improve the chances of a successful treatment. Mammography is the gold standard for breast cancer screening and diagnostic, since it has high sensitivity and high specificity at low cost. Nevertheless the fact of $3 \mathrm{D}$ anatomical information being projected into a $2 \mathrm{D}$ image plane causes some problems as overlapping tissue, loss of sensibility and specificity between tissues. This can mask a tumor or show a false-positive that will result on a recall. The possibility of $3 \mathrm{D}$ reconstruction of mammograms will solve these problems: increases sensibility and specificity, allowing distinguish malignant from benign tissue; allows the 3D visualization without overlapping tissues; reduces recalls and its costs, and assesses the shape, size and location of a lesion.
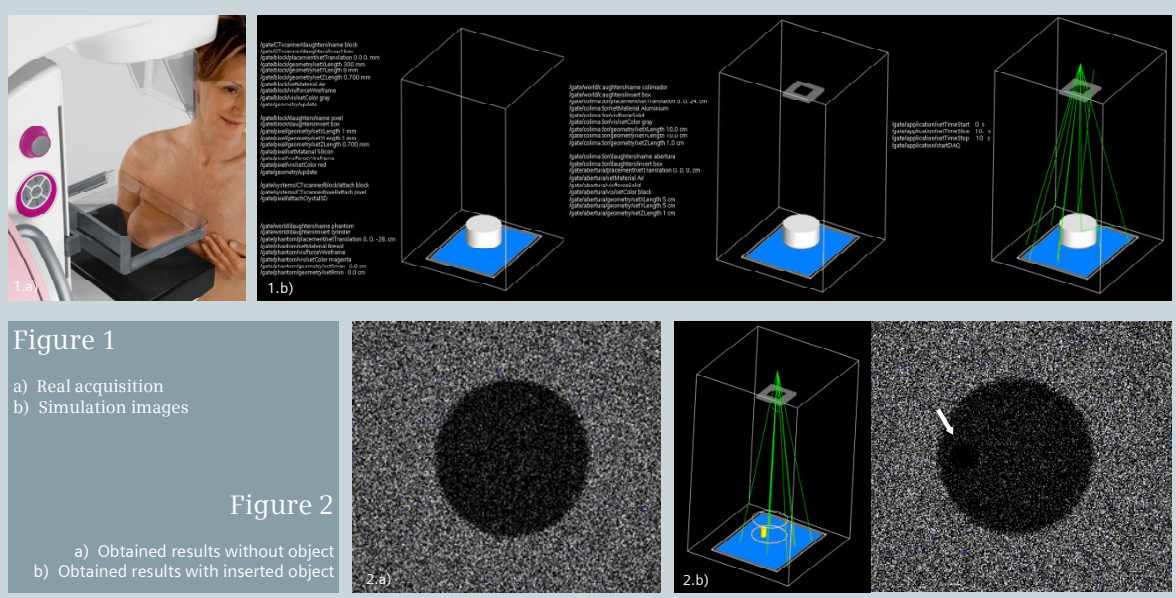

\section{Methodology}

Developed by CERN, Geant4 is a platform created for simulation of interactions between elementary particles and matter. The software is used on different areas of Physics, including Medical Physics. Here, the Monte Carlo

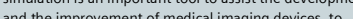
and the improvement of medical asaging devices, 10 assess in the scope of this project the GATE (Geant4 Application for Tomographic Emission) software is used to specifically simulate the acquisition of medical images, according to the introduced parameters. Considering the stochastic nature of the process such as the interaction between the $X$-rays with tissues, the $X$-ray generation and detection, a tool as Monte Carlo methods is important to evaluate the reconstruction of the mammograms.

mammograms.

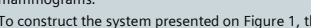
different units were defined on a macro file: - Detector, - Pixels, - Phantom, Collimator, According to: According to: Geometry, - Dimension, - Material,

characteristics relative to each element. A is is showed on Figure 1.

incident photons in the seen On Figure 2 b) a small cylind material was inserted on the phantom constituted by

To simulate mammography data on the CTscanner (one of the system types available in the GATE platform), all system was constructed based on characteristics of a mammography unit and breast tissue. Not all parame are equal to the reality. Some need to be improved accordingly to the real parameters of a mammography breast tissue. Here one can see the different attenuation

On the simulated projection data, algorithms for threedimensional reconstruction will be applied: - Cone-beam algorithm Fan-beam algorithm.
The results will be compared with similar reconstruction on real mammograms:

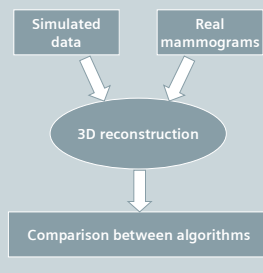

The reconstruction will be performed on STIR (Software for Tomographic Image Reconstruction).

\section{Bibliography}

S. Jan et all (2008). GATE Users Guide. Available on: http:/lopengatecollaboration.healthgrid.org/. D. H. Wright (2005). Physics Reference Manual. Available
on: http://geant4.cern.ch/G4UsersDocuments!. K. Thielemans, S. Mustafovic, C. Tsoumpas (2006). STIR. Software for Tomographic Image Reconstruction Release
2. Nuclear Science Symposium Conference Record, IEEE. 